SUPPORTING INFORMATION

\title{
SYNTHESIS OF THE BCD TRICYCLIC CORE OF DENSANINS A AND B
}

Shan Shi, Hongwei Shi, JianLong Li, Fei Li, Lei Chen, Chao Zhang, Zhiyan Huang, Na Zhao, Nan Li, Jun Yang* 
General Procedures S-1

Experimental Procedures

S-1

Compound 4 S-1

Compound 3 and 5 S-1

Compound 3a S-1

Compound $6 \quad$ S-2

Compound $7 \quad$ S-2

Compound $8 \quad$ S-2

Compound $10 \quad$ S-3

Compound 11 S-3

Compound 13 S-3

Compound 13a S-4

Compound 14 S-4

Compound 15 and $16 \quad$ S-4

Compound 12 S-5

Compound 2 S-5

Compound 17 S-5

Compound 18 S-6

Compound 19 S-6

Compound 20 S-7

Compound 1 S-7

Compound $3{ }^{1} \mathrm{H}$ NMR $\quad$ S-8

Compound $3{ }^{13} \mathrm{C}$ NMR $\quad$ S-9

Compound 3 DEPT S-10

Compound 3 HMQC S-11

Compound 3 NOEDS S-12

Compound $5{ }^{1} \mathrm{H}$ NMR $\quad$ S-13

Compound $5{ }^{13}$ CNMR S-14

Compound 5 DEPT S-15

Compound 5 HMQC S-16

Compound 5 NOEDS $\quad$ S-17

Compound 3a ${ }^{1} \mathrm{H}$ NMR $\quad$ S-18

Compound 3a ${ }^{13} \mathrm{C}$ NMR $\quad$ S-19

Compound $6{ }^{1} \mathrm{H}$ NMR $\quad \mathrm{S}-20$

Compound $6{ }^{13} \mathrm{C}$ NMR $\quad \mathrm{S}-21$

Compound $7{ }^{1} \mathrm{H}$ NMR S-22

Compound $7{ }^{13} \mathrm{C}$ NMR $\quad \mathrm{S}-23$

Compound 7 DEPT $\quad \mathrm{S}-24$

Compound 7 HMQC S-25

Compound $8{ }^{1} \mathrm{H}$ NMR $\quad \mathrm{S}-26$

Compound $8{ }^{13} \mathrm{C}$ NMR $\quad \mathrm{S}-27$

Compound $10{ }^{1} \mathrm{H}$ NMR S-28

Compound $10{ }^{13} \mathrm{C}$ NMR $\quad$ S-29

Compound $11{ }^{1} \mathrm{H}$ NMR S-30

Compound $11{ }^{13} \mathrm{C}$ NMR S-31

Compound 11 DEPT S-32

Compound 11 HMQC S-33

Compound 11 NOEDS S-34

Compound $13{ }^{1} \mathrm{H}$ NMR $\quad \mathrm{S}-35$

Compound $13{ }^{13} \mathrm{C}$ NMR S-36

Compound 13a ${ }^{1} \mathrm{H}$ NMR $\quad \mathrm{S}-37$

Compound 13a ${ }^{13} \mathrm{C}$ NMR $\quad$ S-38

Compound $14{ }^{1} \mathrm{H}$ NMR S-39

Compound $14{ }^{13} \mathrm{C}$ NMR $\quad \mathrm{S}-40$

Compound $15{ }^{1} \mathrm{H}$ NMR S-41

Compound $15{ }^{13} \mathrm{C}$ NMR $\quad \mathrm{S}-42$

Compound 15 DEPT S-43

Compound 15 HMQC S-44

Compound 15 NOEDS S-45

Compound $16{ }^{1} \mathrm{H}$ NMR S-46

Compound $16{ }^{13} \mathrm{C}$ NMR $\quad \mathrm{S}-47$

Compound 16 DEPT S-48

Compound 16 HMQC S-49

Compound 16 NOEDS S-50

Compound $12{ }^{1} \mathrm{H}$ NMR S-51 
Compound $12{ }^{13} \mathrm{C}$ NMR

Compound $2{ }^{1} \mathrm{H}$ NMR

Compound $2{ }^{13} \mathrm{C}$ NMR

Compound $17{ }^{1} \mathrm{H}$ NMR

Compound $17{ }^{13} \mathrm{C}$ NMR

Compound $18{ }^{1} \mathrm{H}$ NMR

Compound $18{ }^{13} \mathrm{C}$ NMR

Compound $19{ }^{1} \mathrm{H}$ NMR

Compound $19{ }^{13} \mathrm{C}$ NMR

Compound $20{ }^{1} \mathrm{H}$ NMR

Compound $20{ }^{13} \mathrm{C}$ NMR

Compound $1{ }^{1} \mathrm{H}$ NMR

Compound $1{ }^{13} \mathrm{C}$ NMR
S-52

S-53

S-54

S-55

S-56

S-57

S-58

S-59

S-60

S-61

S-62

S-63

S-64 
General Procedures:

All reactions were performed using flame-dried round-bottomed flasks or reaction vessels unless otherwise stated. Where appropriate, reactions were carried out under an inert atmosphere of nitrogen with dry solvents, unless otherwise stated. Yields refer to chromatographically, unless otherwise stated. Reagents were purchased at the highest commercial quality and used without further purification, unless otherwise stated. Reactions were monitored by thin-layer chromatography carried out on 0.20-0.25 mm Qingdao Marine Chemical Plant silica gel plates $\left(\mathrm{GF}_{254}\right)$ using ultraviolet light as visualizing agent and an acidic mixture of $p$-anisaldehyde or a basic mixture of potassium permanganate and heat as developing agents. NMR spectra were recorded on a Bruker- $600 \mathrm{~Hz}, \mathrm{Bruker}-400 \mathrm{~Hz}$ or Bruker Avance $300 \mathrm{MHz}$ spectrometer. IR spectra were recorded on a Bruker Tensor 27. High resolution mass spectra (HRMS) dates were recorded on Bruker Apex IV FTMS with ESI source and Bruker Maxis with EI source. Melting points were recorded on a Fisher-Johns 12-144 melting point apparatus. X-ray facility collected and analyzed all X-ray diffraction data were recorded on Bruker Smart Apex II CCD.

Experimental Procedures

Compound 4:

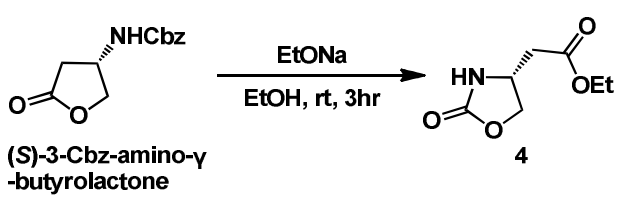

Experimental: Freshly prepared NaOEt/EtOH $(1.0 \mathrm{M}, 85.0 \mathrm{~mL}, 85.0 \mathrm{mmol}, 2.5$ equiv) was added $(S)$-3-Cbz-amino- $\gamma$-butyrolactone $(8.0$ g, $34.0 \mathrm{mmol}, 1.0$ equiv) in dry EtOH $(170 \mathrm{~mL})$ at room temperature. After $3 \mathrm{~h}, \mathrm{HCl}(1.0 \mathrm{M}, 85.0 \mathrm{~mL})$ was added and the reaction solution was evaporated to remove the solvent. The residue was dissolved with EtOAc $(100 \mathrm{~mL})$ and the solid was filtered and washed with EtOAc $(50 \mathrm{~mL} \times 2)$. The combined organic layer was dried over sodium sulfate, filtered and concentrated in vacuum. The residue was then purified with flash chromatography on silica gel (3:1 Hexanes:EtOAc to 1:1 Hexanes:EtOAc) to give oxazolidinone 4 (5.3 g, 90.0\%) as a clear, colorless thick oil. Note: the purity of $(S)$-3-Cbz-amino- $\gamma$-butyrolactone affects the yield of this reaction greatly and oxazolidine 4 is a little water soluble.The NMR data of this compound is consistent with the data reported in the literature (A. I. Franklin, D. Bensa, H. Adams, I. Coldham, Org. Biomol. Chem., 2011, 9, 1901).

Compound $\mathbf{3}$ and 5:

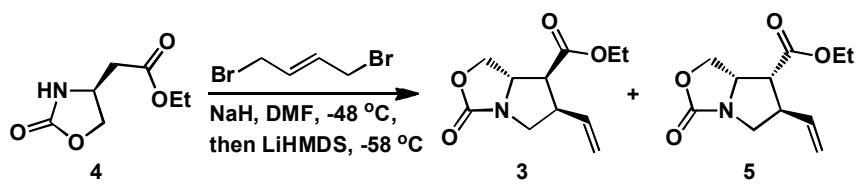

Experimental: Under nitrogen atmosphere, sodium hydride (2.2 g, 60\% in mineral oil, $54.5 \mathrm{mmol}, 1.8$ equiv) was added in dry DMF (300 $\mathrm{mL}$ ) and cooled to $-48{ }^{\circ} \mathrm{C}$. Compound $4(5.3 \mathrm{~g}, 31.2 \mathrm{mmol}, 1.0$ equiv) was dissolved in $50 \mathrm{~mL}$ dry DMF and slowly injected into above solution. The reaction mixture was allowed to stir at the same temperature for $30 \mathrm{~min}$ before a solution of $(E)$-1, 4-dibromo-2-butene (8.0 g, $37.4 \mathrm{mmol}, 1.2$ equiv) in $50 \mathrm{~mL}$ dry DMF was slowly added. The mixture was stirred at $-48{ }^{\circ} \mathrm{C}$ until TLC indicated consumption of the starting material ( 3 hours). The reaction solution was then allowed to cool to $-58^{\circ} \mathrm{C}$ and LiHMDS $(52.0 \mathrm{~mL}$ of a $1.2 \mathrm{M}$ solution in THF, 2.0 equiv) was added in three portions ( 1.0 eq., 0.5 equiv ( 2 hours later), 0.5 equiv ( 5 hours later)). The TLC indicated consumption of the intermediate after 6 hours. The reaction was quenched by pour the mixture into a solution of saturated aqueous ammonia chloride $(400 \mathrm{~mL})$ and EtOAc $(200 \mathrm{~mL})$. The aqueous was extracted with EtOAc $(200 \mathrm{~mL})$, the combined organic layer was dried over sodium sulfate and concentrated in vacuum. The residue was purified via flash chromatography on silica gel $\left(1: 1 \mathrm{Et}_{2} \mathrm{O}: \mathrm{Hexanes}\right)$ to give $30: 1$ mixture of ethyl ester $\mathbf{3}(4.0 \mathrm{~g}, 60.0 \%)$ and $\mathbf{5}$ as a colorless thick oil. The configuration of $\mathbf{3}$ and $\mathbf{5}$ were determined by NOEDS experiment. (for details, see $\mathrm{S}-12, \mathrm{~S}-17)$

Major diastereomer 3: $\mathbf{R}_{\mathbf{f}}=\left(2: 1\right.$ Hexanes:EtOAc) $0.76 .{ }^{1} \mathbf{H}$ NMR: $\left(600 \mathrm{MHz}, \mathrm{CDCl}_{3}\right) \delta 5.68-5.74(\mathrm{~m}, 1 \mathrm{H}), 5.12-5.16(\mathrm{~m}, 2 \mathrm{H}), 4.54-4.56$ $(\mathrm{dd}, J=10.9,7.9 \mathrm{~Hz}, 1 \mathrm{H}), 4.30-4.32(\mathrm{~m}, 1 \mathrm{H}), 4.09-4.19(\mathrm{~m}, 3 \mathrm{H}), 3.90-3.93(\mathrm{dd}, J=11.9,7.8 \mathrm{~Hz}, 1 \mathrm{H}), 3.25-3.30(\mathrm{~m}, 1 \mathrm{H}), 3.12-3.15(\mathrm{dd}, J$ $=11.9,4.5 \mathrm{~Hz}, 1 \mathrm{H}), 2.84-2.87(\mathrm{t}, J=9.0 \mathrm{~Hz}, 1 \mathrm{H}), 1.22-1.24 \mathrm{ppm}(\mathrm{t}, J=9.0 \mathrm{~Hz}, 3 \mathrm{H}) .{ }^{13} \mathbf{C} \mathbf{N M R}:\left(150 \mathrm{MHz}, \mathrm{CDCl}_{3}\right) \delta 169.9,161.3,134.6$, 118.5, 66.9, 61.1, 59.7, 52.6, 51.3, 46.1, 14.2 ppm. IR ( $\left.v_{\max }\right): 1760,1630,1392,1189,1106,1012 \mathrm{~cm}^{-1}$. MS: HRMS (ESI) m/z: Calculated for $\mathrm{C}_{11} \mathrm{H}_{15} \mathrm{NNaO}_{4}(\mathrm{M}+\mathrm{Na})^{+} 248.0893$, found 248.0899. OR: $[\alpha]_{\mathrm{D}}{ }^{20}=\left(\mathrm{c} 1.00, \mathrm{CH}_{2} \mathrm{Cl}_{2}\right)+26.9^{\circ}$

Minor diastereomer 5: $\mathbf{R}_{\mathbf{f}}=\left(4: 1\right.$ Hexanes:EtOAc) $0.75 .{ }^{1} \mathbf{H}$ NMR: $\left(600 \mathrm{MHz}, \mathrm{CDCl}_{3}\right) \delta 5.72-5.78(\mathrm{~m}, 1 \mathrm{H}), 5.12-5.17(\mathrm{~m}, 2 \mathrm{H}), 4.46-4.49$ $(\mathrm{m}, 1 \mathrm{H}), 4.28-4.31(\mathrm{dd}, J=8.0,4.2 \mathrm{~Hz}, 1 \mathrm{H}), 4.14-4.19(\mathrm{~m}, 3 \mathrm{H}), 3.98-4.01(\mathrm{dd}, J=11.6,7.7 \mathrm{~Hz}, 1 \mathrm{H}), 3.20-3.22(\mathrm{~m}, 1 \mathrm{H}), 3.01-3.04(\mathrm{dd}, J=$ 11.6, 6.1 Hz, 1H), 2.87-2.89 (dd, $J=7.7,5.1 \mathrm{~Hz}, 1 \mathrm{H}), 1.24-1.27 \mathrm{ppm}(\mathrm{t}, J=7.2 \mathrm{~Hz}, 3 \mathrm{H}) .{ }^{13} \mathbf{C}$ NMR: $\left(150 \mathrm{MHz}^{-} \mathrm{CDCl}_{3}\right) \delta 170.9,160.9$, 136.5, 117.0, 64.9, 61.5, 59.5, 52.2, 51.1, 46.6, 14.0 ppm. IR $\left(v_{\max }\right): 1766,1639,1340,1192,1113,1009 \mathrm{~cm}^{-1}$. MS: HRMS (ESI) m/z: Calculated for $\mathrm{C}_{11} \mathrm{H}_{15} \mathrm{NNaO}_{4}(\mathrm{M}+\mathrm{Na})^{+} 248.0893$, found 248.0898. OR: $[\alpha]_{\mathrm{D}}{ }^{20}=\left(\mathrm{c} 1.80, \mathrm{CH}_{2} \mathrm{Cl}_{2}\right)+14.8^{\circ}$

Compound 3a:

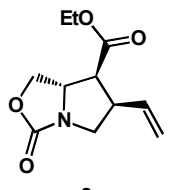

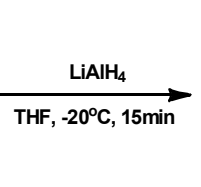

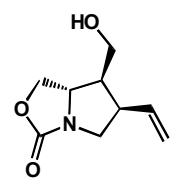

$3 a$

Experimental: Ester 3 (4.0 g, $17.8 \mathrm{mmol}, 1.0$ equiv) was dissolved in dry THF (100 mL) and kept under nitrogen atmosphere. The reaction was cooled to $-20{ }^{\circ} \mathrm{C}, \mathrm{LiAlH}_{4}(3.5 \mathrm{M}$ in Toluene/THF, $8.9 \mathrm{~mol}, 2.6 \mathrm{~mL}, 0.5$ equiv) was added in 10 minutes. Starting material consumed after $15 \mathrm{~min}$ by TLC analysis. The reaction was then quenched by addition of drops of water, and then filtered over a Celite plug, washed with acetone. The filtrate was concentrated under vacuum, then purified via flash chromatography on silica gel (1:1 EtOAc:Hexanes) to give 3a (2.9 g, 90.2\%) as a clear, colorless thick oil. 
$\mathbf{R}_{\mathbf{f}}=\left(100 \%\right.$ EtOAc) 0.60. ${ }^{1} \mathbf{H}$ NMR: $\left(400 \mathrm{MHz}, \mathrm{CDCl}_{3}\right) \delta 5.69-5.78(\mathrm{~m}, 1 \mathrm{H}), 5.08-5.12(\mathrm{~m}, 2 \mathrm{H}), 4.51-4.55(\mathrm{dd}, J=9.0,8.1 \mathrm{~Hz}, 1 \mathrm{H}), 4.29-$ $4.32(\mathrm{dd}, J=9.1,3.8 \mathrm{~Hz}, 1 \mathrm{H}), 3.74-3.93(\mathrm{~m}, 3 \mathrm{H}), 3.50-3.56(\mathrm{~m}, 1 \mathrm{H}), 3.02-3.08(\mathrm{~m}, 2 \mathrm{H}), 2.48-2.50(\mathrm{t}, J=4.4 \mathrm{~Hz}, 1 \mathrm{H}), 2.11-2.19 \mathrm{ppm}(\mathrm{m}$,

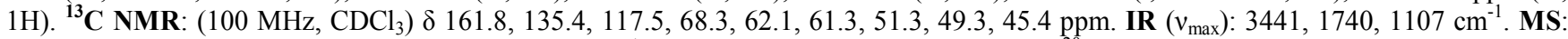
HRMS (ESI) m/z: Calculated for $\mathrm{C}_{9} \mathrm{H}_{13} \mathrm{NNaO}_{3}(\mathrm{M}+\mathrm{Na})^{+} 206.0788$, found 206.0782. OR: $[\alpha]_{\mathrm{D}}{ }^{20}=\left(\mathrm{c} 1.00, \mathrm{CH}_{2} \mathrm{Cl}_{2}\right)+16.1^{\circ}$

Compound 6:

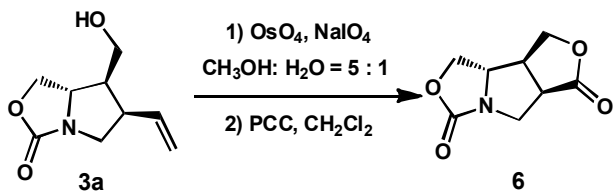

Experimental: Alcohol 3a ( $0.3 \mathrm{~g}, 1.6 \mathrm{mmol}, 1.0$ equiv) was dissolved in $\mathrm{MeOH} / \mathrm{H}_{2} \mathrm{O}(5: 1,60 \mathrm{~mL})$ followed by addition of sodium periodate $(1.1 \mathrm{~g}, 4.9 \mathrm{mmol}, 3.0$ equiv) and $1 \%$ aqueous solution of osmium tetroxide $(2.5 \mathrm{~mL}, 0.1 \mathrm{mmol}, 6 \%$ equiv). The mixture was stirred at $0{ }^{\circ} \mathrm{C}$ for $30 \mathrm{~min}$ before raise to room temperature and stirred for 3 hours. The resulting white slurry form was filtered over a Celite plug, the filter cake was washed with acetone, and then the filtrate was concentrated in vacuum. This crude hemiacetal was pure enough for further use. The crude hemiacetal was dissolved in dry DCM $(25 \mathrm{~mL})$ and pyridinium chlorochromate $(0.7 \mathrm{~g}, 3.3 \mathrm{mmol}, 2.0$ equiv) was added. The reaction was allowed to stir at room temperature for 3 hours. The mixture was filtered over a Celite plug, washed with EtOAc and the filtrate was concentrated under vacuum. The residue was purified via flash chromatography on silica gel (2:1 Hexanes:EtOAc) to afford lactone $6(260.0 \mathrm{mg}, 85.6 \%)$ as a white solid. The configuration of 6 was determined by X-ray.

$\mathbf{R}_{\mathbf{f}}=\left(1.5: 1\right.$ Hexanes:EtOAc) 0.38. ${ }^{1} \mathbf{H N M R}:\left(400 \mathrm{MHz}, \mathrm{CDCl}_{3}\right) \delta$ 4.56-4.60 (dd, $\left.J=9.1,7.4 \mathrm{~Hz}, 1 \mathrm{H}\right), 4.46-4.50(\mathrm{dd}, J=10.1,5.7 \mathrm{~Hz}, 1 \mathrm{H})$, $4.28-4.32(\mathrm{~m}, 2 \mathrm{H}), 4.14-4.19(\mathrm{dd}, J=12.3,9.6 \mathrm{~Hz}, 1 \mathrm{H}), 3.76-3.79(\mathrm{~m}, 1 \mathrm{H}), 3.51-3.55(\mathrm{dd}, J=12.5,3.2 \mathrm{~Hz}, 1 \mathrm{H}), 3.42-3.48(\mathrm{td}, J=9.3,3.2$ $\mathrm{Hz}, 1 \mathrm{H}), 2.82-2.88 \mathrm{ppm}(\mathrm{m}, 1 \mathrm{H}) .{ }^{13} \mathbf{C}$ NMR: $\left(100 \mathrm{MHz}, \mathrm{CDCl}_{3}\right) \delta 176.8,160.4,68.8,66.1,63.1,48.5,45.4,45.2 \mathrm{ppm} . \mathbf{I R}\left(v_{\max }\right): 2917$, 1749, 1589, 1400, 1257, 1165, 1078, 1030, $798 \mathrm{~cm}^{-1}$. MS: HRMS (ESI) m/z: Calculated for $\mathrm{C}_{8} \mathrm{H}_{9} \mathrm{NNaO}_{4}\left(\mathrm{M}+\mathrm{Na}^{+} 206.0424\right.$, found 206.0428. MP: $165-166^{\circ} \mathrm{C}$. OR: $[\alpha]_{\mathrm{D}}{ }^{20}=\left(\mathrm{c} 1.00, \mathrm{CH}_{2} \mathrm{Cl}_{2}\right)+52.8^{\circ}$. X-ray: for coordinates see attached CIF file "xb8720.cif". CCDC deposition number: 1433743

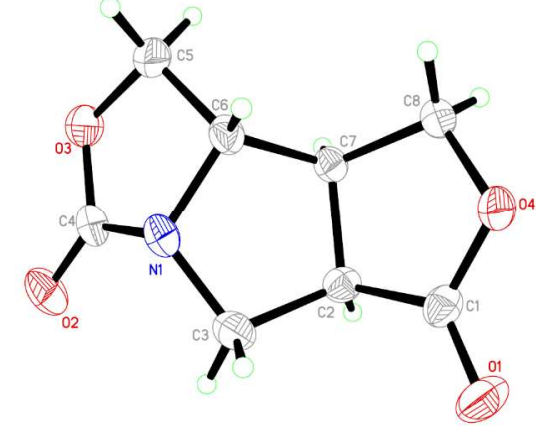

Compound 7:

Experimental: Under nitrogen atmosphere, compound 6 (1.6 g, $8.74 \mathrm{mmol}, 1.0$ equiv) was dissolved in dry THF (40 mL) and cooled to $78{ }^{\circ} \mathrm{C}$ and LiHMDS (21.8 $\mathrm{mL}$ of a $1.2 \mathrm{M}$ solution in THF, 3.0 equiv) was added dropwise. The mixture was stirred at $-78{ }^{\circ} \mathrm{C}$ for 30 min, then allyl bromide $(3.2 \mathrm{~g}, 26.20 \mathrm{mmol}, 3.0$ equiv) until TLC indicated consumption of the starting material ( 3 hours). The reaction was then quenched by addition of 2 eq. acetic acid and saturated aqueous ammonia chloride solution $(100 \mathrm{~mL})$. The mixture was extracted with EtOAc $(3 \times 100 \mathrm{~mL})$. The combined organic extracts were washed with brine, dried over $\mathrm{Na}_{2} \mathrm{SO}_{4}$, concentrated in vacuum. The residue was purified via flash chromatography on silica gel (6:1 Hexanes:EtOAc) to give $7(1.2 \mathrm{~g}, 61.5 \%)$ as a clear, thick oil.

$\mathbf{R}_{\mathbf{f}}=\left(3: 1 \mathrm{Hexanes}:\right.$ EtOAc) $0.38 .{ }^{1} \mathbf{H}$ NMR: $\left(600 \mathrm{MHz}, \mathrm{CDCl}_{3}\right) \delta 5.68-5.75(\mathrm{~m}, 1 \mathrm{H}), 5.23-5.26(\mathrm{~m}, 2 \mathrm{H}), 4.53-4.56(\mathrm{dd}, J=9.3,7.4 \mathrm{~Hz}, 1 \mathrm{H})$, 4.33-4.35 (dd, $J=10.1,5.9 \mathrm{~Hz}, 1 \mathrm{H}), 4.27-4.29(\mathrm{dd}, J=9.3,2.0 \mathrm{~Hz}, 1 \mathrm{H}), 4.18-4.20(\mathrm{~d}, J=10.1 \mathrm{~Hz}, 1 \mathrm{H}), 3.83-3.85(\mathrm{~m}, 2 \mathrm{H}), 3.54-3.56(\mathrm{~d}, J$ $=12.7 \mathrm{~Hz}, 1 \mathrm{H}), 2.59-2.62(\mathrm{dd}, J=14.1,7.3 \mathrm{~Hz}, 1 \mathrm{H}), 2.43-2.47(\mathrm{dd}, J=13.9,7.7 \mathrm{~Hz}, 1 \mathrm{H}) .{ }^{13} \mathbf{C ~ N M R}:\left(150 \mathrm{MHz} \mathrm{CDCl}_{3}\right) \delta 178.8,160.2$, $130.9,121.3,67.7,66.3,64.4,57.2,54.0,49.6,39.0$ ppm. IR $\left(v_{\max }\right): 2917,1757,1398,1193,1072,1032,995,929,772,655 \mathrm{~cm}^{-1} . \mathbf{M S}$ HRMS (ESI) m/z: Calculated for $\mathrm{C}_{11} \mathrm{H}_{13} \mathrm{NNaO}_{4}(\mathrm{M}+\mathrm{Na})^{+} 246.0737$ found 246.0741. OR: $[\alpha]_{\mathrm{D}}{ }^{20}=\left(\mathrm{c} 1.50, \mathrm{CH}_{2} \mathrm{Cl}_{2}\right)+60.7^{\circ}$. Compound 8:

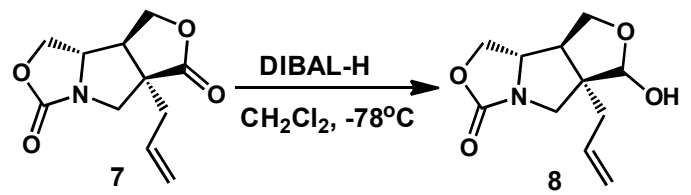

Experimental: Compound 7 ( $0.9 \mathrm{~g}, 3.8 \mathrm{mmol}, 1.0$ equiv) was dissolved in dry $\mathrm{CH}_{2} \mathrm{Cl}_{2}(25 \mathrm{~mL})$ and kept under nitrogen atmosphere. The reaction was cooled to $-78{ }^{\circ} \mathrm{C}$, DIBAl-H (1.0 M in Hexane, $3.8 \mathrm{~mol}, 3.8 \mathrm{~mL}, 1.0$ equiv) was added in 10 minutes. Starting material consumed after $30 \mathrm{~min}$ by TLC analysis. The reaction was then quenched by addition of drops of MeOH followed by the addition of a saturated sodium potassium tartrate solution $(20 \mathrm{~mL})$. The resulting suspension was allowed to warm to room temperature with vigorous stirring for $2 \mathrm{~h}$. The organic layer was collected, and the aqueous layer was extracted with $\mathrm{CH}_{2} \mathrm{Cl}_{2}(3 \times 30 \mathrm{~mL})$. The combined organic fractions 
were washed with brine, dried over $\mathrm{Na}_{2} \mathrm{SO}_{4}$, and concentrated under reduced pressure, and then purified via flash chromatography on silica gel (3:1 EtOAc:Hexanes) to give $\mathbf{8}(750 \mathrm{mg}, 87.6 \%)$ as a clear, colorless thick oil.

Compound $\mathbf{8}$ is a mixture of diastereoisomers. $\mathbf{R}_{\mathbf{f}}=\left(1.5: 1\right.$ Hexanes:EtOAc) $0.32 .{ }^{1} \mathbf{H}$ NMR: $\left(600 \mathrm{MHz}, \mathrm{CDCl}_{3}\right) \delta 5.80-5.86(\mathrm{~m}, 1 \mathrm{H}), 5.71-$ $5.76(\mathrm{~m}, 1 \mathrm{H}), 5.14-5.23(\mathrm{~m}, 6 \mathrm{H}), 4.55-4.58(\mathrm{t}, J=8.6 \mathrm{~Hz}, 1 \mathrm{H}), 4.46-4.48(\mathrm{t}, J=8.2 \mathrm{~Hz}, 1 \mathrm{H}), 4.23-4.25(\mathrm{dd}, J=9.3,2.0 \mathrm{~Hz}, 1 \mathrm{H}), 4.17-4.20$ $(\mathrm{dd}, J=9.1,3.5 \mathrm{~Hz}, 1 \mathrm{H}), 4.11-4.13(\mathrm{dd}, J=9.0,2.8 \mathrm{~Hz}, 1 \mathrm{H}), 3.99-4.08(\mathrm{~m}, 3 \mathrm{H}), 3.88-3.90(\mathrm{dd}, J=9.4,3.0 \mathrm{~Hz}, 1 \mathrm{H}), 3.82-3.85(\mathrm{~m}, 1 \mathrm{H})$, $3.71-3.72(\mathrm{~d}, J=9.2 \mathrm{~Hz}, 1 \mathrm{H}), 3.56-3.64(\mathrm{~m}, 2 \mathrm{H}), 3.16-3.17(\mathrm{~d}, J=2.2 \mathrm{~Hz}, 1 \mathrm{H}), 2.79-2.82(\mathrm{~m}, 2 \mathrm{H}), 2.51-2.54(\mathrm{dd}, J=13.7,5.1 \mathrm{~Hz}, 1 \mathrm{H})$, 2.31-2.37 (m, 3H), 2.23-2.26 (dd, $J=14.0,7.2 \mathrm{~Hz}, 1 \mathrm{H}), 2.12-2.17(\mathrm{~m}, 1 \mathrm{H}) .{ }^{13} \mathbf{C}$ NMR: $\left(150 \mathrm{MHz}, \mathrm{CDCl}_{3}\right) \delta 161.7,160.7,134.3,132.9$, $119.7,119.7,100.8,100.8,70.0,68.6,68.0,67.5,66.6,65.0,63.5,62.8,56.4,54.2,54.0,50.1,40.7,38.0$ ppm. IR $\left(v_{\max }\right): 3419,2921,1939$, 1639, 1472, 1405, 1219, 1075, 108, 920, 769, $629 \mathrm{~cm}^{-1}$. MS: HRMS (ESI) m/z: Calculated for $\mathrm{C}_{11} \mathrm{H}_{15} \mathrm{NNaO}{ }_{4}(\mathrm{M}+\mathrm{Na})^{+} 248.0893$ found 248.0893.

Compound 10:

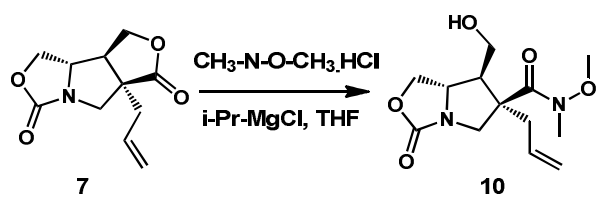

Experimental: In the nitrogen-filled glove box, compound 7 (1.3 g, $5.8 \mathrm{mmol}, 1.0$ equiv) was dissolved in dry DCM (30 mL), then the N,O-Dimethylhydroxylamine hydrochloride $(909.1 \mathrm{mg}, 9.3 \mathrm{mmol}, 1.6$ equiv) was added. The mixture was stirred for $30 \mathrm{~min}$ and isopropyl magnesium chloride $(2.0 \mathrm{M}$ in THF, $8.7 \mathrm{~mL}, 17.46 \mathrm{mmol}, 3.0$ equiv.) was added dropwise. The reaction was stirred for another 1 hour at which point TLC analysis indicated consumption of the starting material. The reaction was quenched by the addition of saturated aqueous $\mathrm{NH}_{4} \mathrm{Cl}(25 \mathrm{~mL})$ and the aqueous phase was extracted with $\mathrm{CH}_{2} \mathrm{Cl}_{2}(3 \times 30 \mathrm{~mL})$. Organic layers were combined and washed with brine $(30$ $\mathrm{mL}$ ) and then dried over $\mathrm{Na}_{2} \mathrm{SO}_{4}$, filtered and concentrated in vacuum. The crude product was purified via flash chromatography on silica gel (1:1 Hexanes:EtOAc ) to give compound $\mathbf{1 0}(1.5 \mathrm{~g}, 92.0 \%)$ as a clear, colorless thick oil.

$\mathbf{R}_{\mathbf{f}}=\left(1: 1\right.$ Hexanes:EtOAc) 0.25. ${ }^{1} \mathbf{H}$ NMR: $\left(600 \mathrm{MHz}, \mathrm{CDCl}_{3}\right) \delta 5.73-5.80(\mathrm{~m}, 1 \mathrm{H}), 5.20-5.24(\mathrm{~m}, 2 \mathrm{H}), 4.38-4.41(\mathrm{dd}, J=9.5,6.3 \mathrm{~Hz}, 1 \mathrm{H})$, $4.21-4.25(\mathrm{~m}, 2 \mathrm{H}), 4.04-4.06(\mathrm{~d}, J=11.5 \mathrm{~Hz}, 1 \mathrm{H}), 3.71-3.72(\mathrm{~m}, 2 \mathrm{H}), 3.60(\mathrm{~s}, 3 \mathrm{H}), 3.51-3.53(\mathrm{~d}, J=11.6 \mathrm{~Hz}, 1 \mathrm{H}), 3.26(\mathrm{br}, 1 \mathrm{H}), 2.98(\mathrm{~s}$, $3 \mathrm{H}), 2.75-2.77(\mathrm{~m}, 1 \mathrm{H}), 2.51-2.55(\mathrm{dd}, J=13.9,7.1 \mathrm{~Hz}, 1 \mathrm{H}), 2.39-2.43(\mathrm{dd}, J=13.9,7.9 \mathrm{~Hz}, 1 \mathrm{H}) .{ }^{13} \mathbf{C} \mathbf{N M R}:\left(150 \mathrm{MHz}, \mathrm{CDCl}_{3}\right) \delta 179.6$, $160.4,131.5,120.4,71.0,66.6,64.1,59.4,56.3,54.1,44.9,37.8,35.2$ ppm. IR $\left(v_{\max }\right): 3459,2924,2027,1766,1638,1424,1385,1166$, 1034, 926, $611 \mathrm{~cm}^{-1}$. MS: HRMS (ESI) m/z: Calculated for $\mathrm{C}_{13} \mathrm{H}_{20} \mathrm{~N}_{2} \mathrm{NaO}_{5}(\mathrm{M}+\mathrm{Na})^{+} 307.1264$ found 307.1270 .

OR: $[\alpha]_{\mathrm{D}}{ }^{20}=\left(\mathrm{c} 0.60, \mathrm{CH}_{2} \mathrm{Cl}_{2}\right)+21.0^{\circ}$

Compound 11:

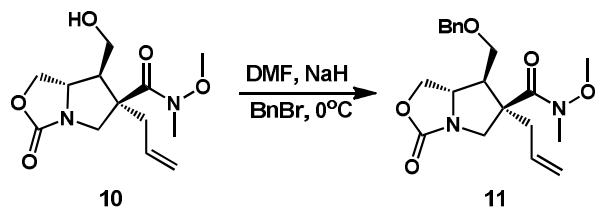

Experimental: Under nitrogen atmosphere, sodium hydride (60\% dispersion in mineral oil, $281.0 \mathrm{mg}, 7.0 \mathrm{mmol}, 2.0$ equiv) was added in dry DMF $(20 \mathrm{~mL})$ and cooled to $0{ }^{\circ} \mathrm{C}$. A solution of $\mathbf{1 0}(1.0 \mathrm{~g}, 3.5 \mathrm{mmol}, 1.0$ equiv. $)$ in dry DMF $(5 \mathrm{~mL})$ was added, the resulting mixture was stirred for $20 \mathrm{~min}$ before benzyl bromide $(0.6 \mathrm{~mL}, 5.3 \mathrm{mmol}, 1.5$ equiv.) was added. The reaction was warmed to room temperature and stirred for another 2 hour at which point TLC analysis indicated consumption of the starting material. The reaction was quenched by the addition of saturated aqueous $\mathrm{NH}_{4} \mathrm{Cl}(25 \mathrm{~mL})$ and the aqueous phase was extracted with EtOAc $(3 \times 50 \mathrm{~mL})$. Organic layers were combined and washed with brine $(30 \mathrm{~mL})$ and then dried over $\mathrm{Na}_{2} \mathrm{SO}_{4}$, filtered, and concentrated in vacuum. The crude product was purified via flash chromatography on silica gel (3:1 Hexanes:EtOAc ) to give compound $11(718.0 \mathrm{mg}, 54.5 \%)$ as a clear, colorless thick oil. The configuration of $\mathbf{1 1}$ was determined by NOEDS experiment. (for details, see S-34)

$\mathbf{R}_{\mathbf{f}}=\left(2: 1\right.$ Hexanes:EtOAc) 0.38. ${ }^{1} \mathbf{H}$ NMR: $\left(600 \mathrm{MHz}, \mathrm{CDCl}_{3}\right) \delta$ 7.26-7.36 (m, 5H), 5.71-5.77 (m, 1H), 5.15-5.18 (m, $\left.2 \mathrm{H}\right), 4.52(\mathrm{~s}, 2 \mathrm{H})$, 4.34-4.37 (m, 2H), 4.17-4.19 (d, $J=9.5 \mathrm{~Hz}, 1 \mathrm{H}), 4.01-4.03(\mathrm{~d}, J=11.3 \mathrm{~Hz}, 1 \mathrm{H}), 3.56-3.63(\mathrm{~m}, 2 \mathrm{H}), 3.55(\mathrm{~s}, 3 \mathrm{H}), 3.49-3.51(\mathrm{~d}, J=11.4 \mathrm{~Hz}$, $1 \mathrm{H}), 2.96(\mathrm{~s}, 3 \mathrm{H}), 2.90-2.91(\mathrm{~m}, 1 \mathrm{H}), 2.50-2.53(\mathrm{dd}, J=13.8,6.8 \mathrm{~Hz}, 1 \mathrm{H}), 2.36-2.40(\mathrm{dd}, J=13.7,8.0 \mathrm{~Hz}, 1 \mathrm{H}) .{ }^{13} \mathbf{C ~ N M R}:(150 \mathrm{MHz}$, $\left.\mathrm{CDCl}_{3}\right) \delta 180.0,159.2,137.9,131.8,128.4,128.4,127.8,127.8,127.6,120.0,73.4,71.6,70.6,65.5,59.0,56.6,54.5,44.9,37.9,35.1 \mathrm{ppm}$. IR $\left(v_{\max }\right): 3459,2920,1768,1647,1417,1383,1164,1105,1036,924,743,698 \mathrm{~cm}^{-1}$. MS: HRMS (ESI) m/z: Calculated for $\mathrm{C}_{20} \mathrm{H}_{26} \mathrm{~N}_{2} \mathrm{NaO}_{5}(\mathrm{M}+\mathrm{Na})^{+} 397.1734$ found 397.1735. OR: $[\alpha]_{\mathrm{D}}{ }^{20}=\left(\mathrm{c} 1.17, \mathrm{CH}_{2} \mathrm{Cl}_{2}\right)-28.8^{\circ}$.

Compound 13:

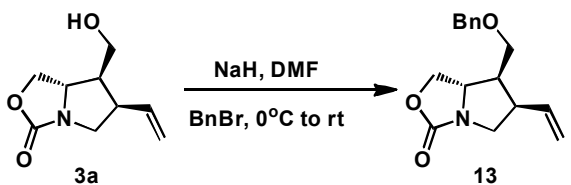

Experimental: Under nitrogen atmosphere, sodium hydride (60\% dispersion in mineral oil, $874.0 \mathrm{mg}, 21.8 \mathrm{mmol}, 2.0$ equiv) was added in dry DMF $(40 \mathrm{~mL})$ and cooled to $0{ }^{\circ} \mathrm{C}$. A solution of $\mathbf{3 a}(2.0 \mathrm{~g}, 10.9 \mathrm{mmol}, 1.0$ equiv) in dry DMF $(5 \mathrm{~mL})$ was added, the resulting mixture was stirred for $20 \mathrm{~min}$ before benzyl bromide $(2.0 \mathrm{~mL}, 16.4 \mathrm{mmol}, 1.5$ equiv) was added. The reaction was warmed to room temperature and stirred for another 2 hour at which point TLC analysis indicated consumption of the starting material. The reaction was quenched by the addition of saturated aqueous $\mathrm{NH}_{4} \mathrm{Cl}(25 \mathrm{~mL})$ and the aqueous phase was extracted with EtOAc $(3 \times 50 \mathrm{~mL})$. Organic layers were combined and washed with brine $(30 \mathrm{~mL})$ and then dried over $\mathrm{Na}_{2} \mathrm{SO}_{4}$, filtered, and concentrated in vacuum. The crude product was purified via flash chromatography on silica gel (4:1 Hexanes:EtOAc ) to give compound $\mathbf{1 3}(2.6 \mathrm{~g}, 85.5 \%)$ as a clear, colorless thick oil. $\mathbf{R}_{\mathbf{f}}=(1: 1 \mathrm{Hexanes:EtOAc})$ 0.52. ${ }^{1} \mathbf{H}$ NMR: $\left(400 \mathrm{MHz}, \mathrm{CDCl}_{3}\right) \delta$ 7.26-7.38 (m, 5H), 5.67-5.76 (m, 1H), 5.08-5.12 (m, 2H), 4.47-4.54 (m, $3 \mathrm{H}), 4.30-4.34(\mathrm{dd}, J=9.1,3.7 \mathrm{~Hz}, 1 \mathrm{H}), 3.86-3.92(\mathrm{~m}, 2 \mathrm{H}), 3.56-3.60(\mathrm{dd}, J=9.4,4.8 \mathrm{~Hz}, 1 \mathrm{H}), 3.35-3.40(\mathrm{t}, J=9.2 \mathrm{~Hz}, 1 \mathrm{H}), 3.06-3.10(\mathrm{~m}$, 
2H), 2.23-2.27 ppm (m, 1H). ${ }^{13} \mathbf{C}$ NMR: $\left(100 \mathrm{MHz}, \mathrm{CDCl}_{3}\right) \delta 161.5,137.8,135.3,128.4,128.4$ 127.7, 127.3, 127.3, 117.4, 73.3, 69.2, 68.0, 62.4, 51.4, 47.2, 45.5 ppm. IR $\left(v_{\max }\right): 1755,1637,1394,1101 \mathrm{~cm}^{-1} . \mathbf{M S}$ : HRMS (ESI) m/z: Calculated for $\mathrm{C}_{16} \mathrm{H}_{19} \mathrm{NNaO} \mathrm{Na}_{3}(\mathrm{M}+\mathrm{Na})^{+}$ 296.1257, found 296.1260. OR: $[\alpha]_{\mathrm{D}}^{20}=\left(\mathrm{c} 1.00, \mathrm{CH}_{2} \mathrm{Cl}_{2}\right)+10.5^{\circ}$.

Compound 13a:

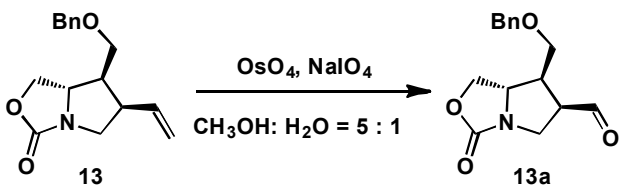

Experimental: Compound $\mathbf{1 3}$ (2.3 g, $8.4 \mathrm{mmol}, 1.0$ equiv) was dissolved in aqueous $\mathrm{MeOH}\left(\mathrm{MeOH} / \mathrm{H}_{2} \mathrm{O}=5 / 1,200 \mathrm{~mL}\right)$. Sodium periodate (5.4 g, $25.2 \mathrm{mmol}, 3.0$ equiv) was added followed by $1 \%$ aqueous solution of osmium tetroxide (128.0 mg, $12.8 \mathrm{mmol}, 0.06 \mathrm{equiv})$. The reaction was stirred at $0{ }^{\circ} \mathrm{C}$ for $30 \mathrm{~min}$, then warmed to $20{ }^{\circ} \mathrm{C}$ and stirred for another 3 hours until TLC indicated consumption of the starting material. The mixture was filtered over a Celite plug. The filtrate was concentrated under vacuum, and extracted with EtOAc $(3 \times$ $50 \mathrm{~mL}$ ). The organic extracts were combined, dried over $\mathrm{Na}_{2} \mathrm{SO}_{4}$, filtered, and concentrated in vacuum. The resulting crude residue was purified via flash chromatography on silica gel (2:1 Hexanes:EtOAc) to 13a (2.2 g, 93.1\%) as a clear, yellow thick oil.

$\mathbf{R}_{\mathbf{f}}=\left(2: 1\right.$ Hexanes:EtOAc) 0.33. ${ }^{1} \mathbf{H}$ NMR: $\left(400 \mathrm{MHz}, \mathrm{CDCl}_{3}\right) \delta 9.74(\mathrm{~s}, 1 \mathrm{H}), 7.26-7.37(\mathrm{~m}, 5 \mathrm{H}), 4.42-4.50(\mathrm{~m}, 3 \mathrm{H}), 4.20-4.24(\mathrm{dd}, J=9.2$, $2.6 \mathrm{~Hz}, 1 \mathrm{H}), 3.82-3.88(\mathrm{~m}, 2 \mathrm{H}), 3.62-3.71(\mathrm{~m}, 3 \mathrm{H}), 3.24-3.32(\mathrm{~m}, 1 \mathrm{H}), 2.44-2.54 \mathrm{ppm}(\mathrm{m}, 1 \mathrm{H}) .{ }^{13} \mathbf{C ~ N M R :}\left(100 \mathrm{MHz}, \mathrm{CDCl}_{3}\right) \delta 200.1$, 161.4, 137.0, 128.6, 128.5, 128.1, 127.7, 127.7, 73.5, 66.8, 62.2, 61.0, 52.0, 47.6, 45.4 ppm. IR $\left(v_{\max }\right): 1750,1630,1388,1100 \mathrm{~cm}^{-1} . \mathbf{M S}$ : HRMS (ESI) $\mathrm{m} / \mathrm{z}$ : Calculated for $\mathrm{C}_{15} \mathrm{H}_{17} \mathrm{NNaO}_{4}(\mathrm{M}+\mathrm{Na})^{+} 298.1050$, found 298.1044. OR: $[\alpha]_{\mathrm{D}}{ }^{20}=\left(\mathrm{c} 0.70, \mathrm{CH}_{2} \mathrm{Cl}_{2}\right)+19.7^{\circ}$

Compound 14

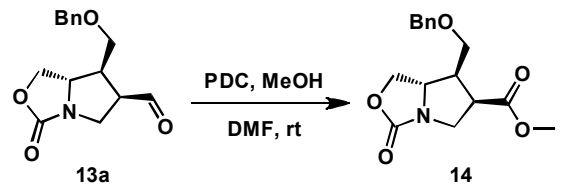

Experimental: Compound 13a (2.0 g, $7.3 \mathrm{mmol}, 1.0$ equiv) was dissolved in $50 \mathrm{~mL}$ dry DMF and $1 \mathrm{~mL}$ dry MeOH. Pyridinium dichromate (5.5 g, $14.6 \mathrm{mmol}, 2.0$ equiv) was added and stirred at room temperature overnight. TLC indicated consumption of the starting material. The mixture was filtered over a Celite plug, and the filter cake was washed with acetone. The combined organics were concentrated under vacuum. The residue was diluted with water $(200 \mathrm{~mL})$ and extracted with EtOAc $(3 \times 60 \mathrm{~mL})$. The organic extracts were combined and washed with brine, dried over $\mathrm{Na}_{2} \mathrm{SO}_{4}$, filtered, and concentrated in vacuum. The resulting crude residue was purified via flash chromatography on silica gel (5:1 Hexanes:EtOAc) to $\mathbf{1 4}(1.9 \mathrm{~g}, 87.3 \%)$ as a clear, yellow thick oil.

$\mathrm{R}_{\mathrm{f}}=\left(2: 1\right.$ Hexanes:EtOAc ) 0.5. ${ }^{1} \mathbf{H}$ NMR: $\left(600 \mathrm{MHz}, \mathrm{CDCl}_{3}\right) \delta$ 7.26-7.38 (m, 5H), 4.47-4.50 (m, 3H), 4.31-4.33 (dd, $\left.J=9.2,2.9 \mathrm{~Hz}, 1 \mathrm{H}\right)$, $3.97-4.01(\mathrm{~m}, 1 \mathrm{H}), 3.91-3.94(\mathrm{dd}, J=12.1,8.3 \mathrm{~Hz}, 1 \mathrm{H}), 3.68-3.71(\mathrm{dd}, J=9.4,5.1 \mathrm{~Hz}, 1 \mathrm{H}), 3.66(\mathrm{~s}, 3 \mathrm{H}), 3.51-3.54(\mathrm{dd}, J=12.1,4.3 \mathrm{~Hz}$, $1 \mathrm{H}), 3.42-3.45(\mathrm{t}, J=8.6 \mathrm{~Hz}, 1 \mathrm{H}), 3.26-3.29(\mathrm{td}, J=8.7,2.2 \mathrm{~Hz}, 1 \mathrm{H}), 2.38-2.42 \mathrm{ppm}(\mathrm{m}, 1 \mathrm{H}) .{ }^{13} \mathbf{C} \mathbf{N M R}:(150 \mathrm{MHz}, \mathrm{CDCl} 3) \delta 172.8$, 161.4, 137.5, 128.5, 128.5, 127.8, 127.4, 127.4, 73.5, 67.9, 67.2, 62.1, 52.0, 48.8, 46.6, 45.5 ppm. IR $\left(v_{\max }\right): 1750,1634,1385,1103 \mathrm{~cm}^{-1}$. MS: HRMS (ESI) m/z: Calculated for $\mathrm{C}_{16} \mathrm{H}_{19} \mathrm{NNaO}_{5}(\mathrm{M}+\mathrm{Na})^{+} 328.1155$, found 328.1164. OR: $[\alpha]_{\mathrm{D}}{ }^{20}=\left(\mathrm{c} 0.83, \mathrm{CH}_{2} \mathrm{Cl}_{2}\right)+35.3^{\circ}$. Compound 15 and 16:
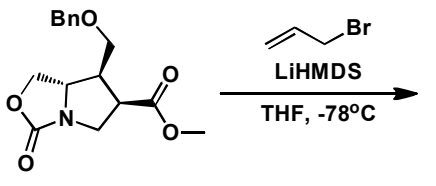

14

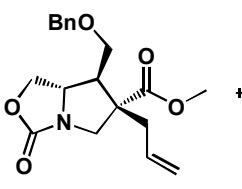

15

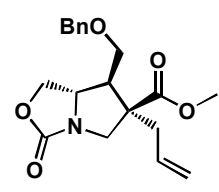

16

Experimental: A solution of $\mathbf{1 4}(1.8 \mathrm{~g}, 5.9 \mathrm{mmol})$ in dry THF $(85 \mathrm{~mL})$ at $-78^{\circ} \mathrm{C}$ was added LiHMDS $(1 \mathrm{M}$ in THF, $17.7 \mathrm{mmol}, 17.7 \mathrm{~mL}$, 3.0 equiv). After stirring for 30 minutes, allyl bromide $(2.1 \mathrm{~g}, 1.5 \mathrm{~mL}, 17.7 \mathrm{mmol}, 3.0$ equiv) was added dropwisely and stirred for another 2 hours. The reaction was then quenched by addition of 2.0 equiv acetic acid and saturated aqueous ammonia chloride solution ( $100 \mathrm{~mL})$. The mixture was extracted with EtOAc $(3 \times 100 \mathrm{~mL})$. The combined organic extracts were washed with brine, dried over $\mathrm{Na}_{2} \mathrm{SO}_{4}$, concentrated in vacuum. The residue was purified via flash chromatography on silica gel (8:1 Hexanes:EtOAc) to give $\mathbf{1 5}(0.5 \mathrm{~g}, 23.5 \%)$ and $\mathbf{1 6}$ $(1.4 \mathrm{~g}, 70.5 \%)$ as a clear, thick oil. The configuration of $\mathbf{1 5}$ and $\mathbf{1 6}$ were determined by NOEDS experiment (for details, see page S-45, S$50)$.

Minor diastereomer 15: $\mathbf{R}_{\mathbf{f}}=\left(4: 1\right.$ Hexanes:EtOAc) $0.33 .{ }^{1} \mathbf{H}$ NMR: $\left(600 \mathrm{MHz}, \mathrm{CDCl}_{3}\right) \delta$ 7.26-7.37 (m, 5H), 5.61-5.66 (m, 1H), 5.07-5.13 $(\mathrm{m}, 2 \mathrm{H}), 4.46-4.53(\mathrm{~m}, 3 \mathrm{H}), 4.33-4.35(\mathrm{dd}, J=9.1,4.0 \mathrm{~Hz}, 1 \mathrm{H}), 4.17-4.19(\mathrm{~d}, J=12.2 \mathrm{~Hz}, 1 \mathrm{H}), 3.97-4.00(\mathrm{~m}, 1 \mathrm{H}), 3.77-3.79(\mathrm{dd}, J=9.2$, $4.3 \mathrm{~Hz}, 1 \mathrm{H}), 3.71(\mathrm{~s}, 3 \mathrm{H}), 3.56-3.58(\mathrm{t}, J=9.0 \mathrm{~Hz}, 1 \mathrm{H}), 3.24-3.26(\mathrm{~d}, J=12.2 \mathrm{~Hz}, 1 \mathrm{H}), 2.50-2.56(\mathrm{~m}, 2 \mathrm{H}), 2.26-2.29 \mathrm{ppm}(\mathrm{dd}, J=14.0,8.0$ $\mathrm{Hz}, 1 \mathrm{H}) .{ }^{13} \mathbf{C}$ NMR: $\left(150 \mathrm{MHz}, \mathrm{CDCl}_{3}\right) \delta 174.3,161.0,137.6,132.8,128.5,128.5,127.9,127.4,127.4,119.5,73.5,68.2,68.1,62.8,55.8$, 53.4, 52.6, 50.8, 36.6 ppm. IR $\left(v_{\max }\right): 1754,1632,1395,1105 \mathrm{~cm}^{-1}$. MS: HRMS (ESI) m/z: Calculated for $\mathrm{C}_{19} \mathrm{H}_{23} \mathrm{NNaO}{ }_{5}(\mathrm{M}+\mathrm{Na})^{+}$ 368.1468 , found 368.1474 . OR: $[\alpha]_{\mathrm{D}}^{20}=\left(\mathrm{c} 1.00, \mathrm{CH}_{2} \mathrm{Cl}_{2}\right)-12.6^{\circ}$.

Major diastereomer 16: $\mathrm{R}_{\mathrm{f}}=\left(4: 1\right.$ Hexanes:EtOAc) $0.34 .{ }^{1} \mathbf{H}$ NMR: $\left(600 \mathrm{MHz}, \mathrm{CDCl}_{3}\right) \delta$ 7.26-7.38 $(\mathrm{m}, 5 \mathrm{H}), 5.66-5.73(\mathrm{~m}, 1 \mathrm{H}), 5.14-5.19$ $(\mathrm{m}, 2 \mathrm{H}), 4.47-4.50(\mathrm{~m}, 3 \mathrm{H}), 4.33-4.35(\mathrm{dd}, J=9.2,3.2 \mathrm{~Hz}, 1 \mathrm{H}), 4.01-4.05(\mathrm{~m}, 1 \mathrm{H}), 3.64-3.73(\mathrm{~m}, 6 \mathrm{H}), 3.34-3.37(\mathrm{t}, J=8.8 \mathrm{~Hz}, 1 \mathrm{H}), 2.75-$ $2.78(\mathrm{dd}, J=13.9,6.2 \mathrm{~Hz}, 1 \mathrm{H}), 2.23-2.26(\mathrm{dd}, J=13.9,8.0 \mathrm{~Hz}, 1 \mathrm{H}), 2.09-2.13 \mathrm{ppm}(\mathrm{dd}, J=8.9,4.3 \mathrm{~Hz}, 1 \mathrm{H}) .{ }^{13} \mathbf{C ~ N M R}:\left(150 \mathrm{MHz}, \mathrm{CDCl}{ }_{3}\right)$ $\delta$ 174.3, 161.2, 137.5, 132.6, 128.4, 128.4, 127.8, 127.3, 127.3, 119.9, 73.4, 67.8, 67.3, 62.7, 56.3, 53.3, 52.6, 52.1, 41.1 ppm. IR (v $\left.v_{\max }\right)$ : 1750, 1634, 1389, $1104 \mathrm{~cm}^{-1}$. MS: HRMS (ESI) m/z: Calculated for $\mathrm{C}_{19} \mathrm{H}_{23} \mathrm{NNaO}_{5}(\mathrm{M}+\mathrm{Na})^{+} 368.1468$, found 368.1475.OR: $[\alpha]_{\mathrm{D}}{ }^{20}=(\mathrm{c}$ $\left.1.67, \mathrm{CH}_{2} \mathrm{Cl}_{2}\right)-22.4^{\circ}$ 
Compound 12:

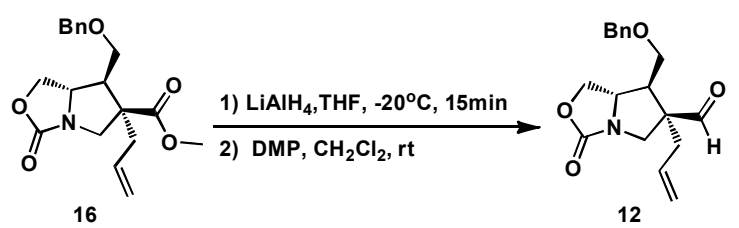

Experimental: Under nitrogen atmosphere, compound $16(0.8 \mathrm{~g}, 2.3 \mathrm{mmol})$ was dissolved in dry $\mathrm{THF}(35 \mathrm{~mL})$ and then cooled to $-20{ }^{\circ} \mathrm{C}$. Lithium aluminum hydride (3.5 M in Toluene/THF, $1.2 \mathrm{~mol}, 0.3 \mathrm{~mL}, 0.5$ equiv) was added dropwisely and then stirred at the same temperature for 15 minutes. The starting material was found to be completely consumed. The reaction was then quenched with $0.3 \mathrm{~mL} \mathrm{H}_{2} \mathrm{O}$, filtered through a Celite plug, washed with acetone. The filtrate was concentrated under vacuum to afford crude alcohol, which was pure enough for further use. To a solution of above crude alcohol in dichloromethane $(50 \mathrm{~mL})$, Dess-Martin periodinane (1.5 g, $3.5 \mathrm{mmol}, 1.5$ equiv.) was added at room temperature. The reaction mixture became cloudy quickly and was stirred for 30 min. TLC analysis indicated consumption of starting material. The solution was quenched with saturated aq. $\mathrm{Na}_{2} \mathrm{~S}_{2} \mathrm{O}_{3}$ and aq. $\mathrm{NaHCO}_{3}$. The aqueous layer was extracted with $\mathrm{CH}_{2} \mathrm{Cl}_{2}(2 \times 100 \mathrm{~mL})$ and the combined organic were washed with saturated aq. $\mathrm{Na}_{2} \mathrm{~S}_{2} \mathrm{O}_{3}$ and aq. $\mathrm{NaHCO}_{3}$, dried with $\mathrm{Na}_{2} \mathrm{SO}_{4}$, filtered, and concentrated in vacuum. The residue was purified via flash chromatography on silica gel (3:1 Hexanes:EtOAc) give compound $12(570.0 \mathrm{mg}, 78.1 \%)$ as a clear, thick oil.

$\mathbf{R}_{\mathbf{f}}=\left(2: 1\right.$ Hexanes:EtOAc) 0.43. ${ }^{1} \mathbf{H}$ NMR: $\left(400 \mathrm{MHz}, \mathrm{CDCl}_{3}\right) \delta 9.61(\mathrm{~s}, 1 \mathrm{H}), 7.24-7.37(\mathrm{~m}, 5 \mathrm{H}), 5.60-5.68(\mathrm{~m}, 1 \mathrm{H}), 5.15-5.18(\mathrm{~m}, 2 \mathrm{H})$, 4.42-4.46 (m, 3H), 4.19-4.21 (dd, $J=9.2,2.7 \mathrm{~Hz}, 1 \mathrm{H}), 3.83-3.86(\mathrm{~m}, 1 \mathrm{H}), 3.67-3.69(\mathrm{~d}, J=12.28 \mathrm{~Hz}, 1 \mathrm{H}), 3.57-3.61(\mathrm{dd}, J=11.6,6.4 \mathrm{~Hz}$, $2 \mathrm{H}), 3.50-3.54(\mathrm{dd}, J=9.8,6.2 \mathrm{~Hz}, 1 \mathrm{H}), 2.69-2.73(\mathrm{dd}, J=14.2,7.8 \mathrm{~Hz}, 1 \mathrm{H}), 2.32-2.37(\mathrm{dd}, J=14.2,7.8 \mathrm{~Hz}, 1 \mathrm{H}), 2.10-2.14 \mathrm{ppm}(\mathrm{m}, 1 \mathrm{H})$. ${ }^{13}$ C NMR: $\left(100 \mathrm{MHz} \mathrm{CDCl}_{3}\right) \delta 202.1,161.2,137.1,131.6,128.6,128.6,128.1,127.6,127.6,120.6,73.6,66.7,65.8,61.5,59.4,53.2$, 50.2, $39.1 \mathrm{ppm}$. IR $\left(v_{\max }\right): 1754,1636,1391,1101 \mathrm{~cm}^{-1}$. MS: HRMS (ESI) m/z: Calculated for $\mathrm{C}_{18} \mathrm{H}_{21} \mathrm{NNaO}_{4}(\mathrm{M}+\mathrm{Na})^{+} 338.1363$, found 338.1370.0R: $[\alpha]_{\mathrm{D}}{ }^{20}=\left(\mathrm{c} 0.70, \mathrm{CH}_{2} \mathrm{Cl}_{2}\right)-12.8^{\circ}$.

Compound 2:
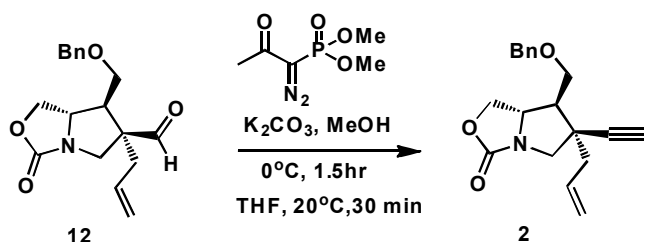

Experimental: Under nitrogen atmosphere, anhydrous $\mathrm{K}_{2} \mathrm{CO}_{3}(709.0 \mathrm{mg}, 5.1 \mathrm{mmol}, 3.0$ equiv) was added to a solution of the OhiraBestmann reagent $\left(1.3 \mathrm{~g}, 6.8 \mathrm{mmol}, 4.0\right.$ equiv) in $\mathrm{MeOH}(45 \mathrm{~mL})$ at $0{ }^{\circ} \mathrm{C}$. After stirring at the same temperature for 1.5 hours, the reaction was heated to $20^{\circ} \mathrm{C}$ and a solution of compound $12(540.0 \mathrm{mg}, 1.7 \mathrm{mmol}, 1.0$ equiv) in dry THF (10 mL) was added over $30 \mathrm{~min}$. The resulting mixture was stirred at $20^{\circ} \mathrm{C}$ for 1 hour; TLC analysis indicated the consumption of starting material. The solid was filtered off, the filter cake was washed with EtOAc, and combined organics were concentrated in vacuum. The residue was purified via flash chromatography on silica gel $(6: 1$ Hexanes:EtOAc) to give compound $2(411.0 \mathrm{mg}, 76.9 \%)$ as a colorless oil.

$\mathbf{R}_{\mathbf{f}}=\left(3: 1\right.$ Hexanes:EtOAc) 0.62. ${ }^{1} \mathbf{H}$ NMR: $\left(400 \mathrm{MHz}, \mathrm{CDCl}_{3}\right) \delta$ 7.26-7.38 (m, 5H), 5.80-5.89 (m, 1H), 5.14-5.19 (m, 2H), 4.47-4.52 (m, $3 \mathrm{H}), 4.37-4.40(\mathrm{dd}, J=8.3,1.4 \mathrm{~Hz}, 1 \mathrm{H}), 3.97-4.02(\mathrm{~m}, 1 \mathrm{H}), 3.76-3.79(\mathrm{dd}, J=9.2,3.4 \mathrm{~Hz}, 1 \mathrm{H}), 3.63-3.72(\mathrm{~m}, 2 \mathrm{H}), 3.41-3.44(\mathrm{~d}, J=11.6$ $\mathrm{Hz}, 1 \mathrm{H}), 2.43-2.48(\mathrm{dd}, J=13.6,6.9 \mathrm{~Hz}, 1 \mathrm{H}), 2.34(\mathrm{~m}, 1 \mathrm{H}), 2.26-2.31(\mathrm{dd}, J=13.6,7.6 \mathrm{~Hz}, 1 \mathrm{H}), 1.95-2.01 \mathrm{ppm}(\mathrm{m}, 1 \mathrm{H}) .{ }^{13} \mathbf{C ~ N M R}:(100$ $\left.\mathrm{MHz}, \mathrm{CDCl}_{3}\right) \delta 160.9,137.6,132.6,128.2,128.2,127.5,127.1,127.1,119.2,84.1,73.7,73.2,69.4,67.4,63.5,57.9,51.4,45.4,43.6$ ppm. IR $\left(v_{\max }\right): 1756,1634,1389,1104,620 \mathrm{~cm}^{-1}$. MS: HRMS (ESI) $\mathrm{m} / \mathrm{z}$ : Calculated for $\mathrm{C}_{19} \mathrm{H}_{21} \mathrm{NNaO}_{3}(\mathrm{M}+\mathrm{Na})^{+} 334.1414$, found 334.1415.OR: $[\alpha]_{\mathrm{D}}^{20}=\left(\mathrm{c} 0.60, \mathrm{CH}_{2} \mathrm{Cl}_{2}\right)+14.0^{\circ}$.

Compound 17:

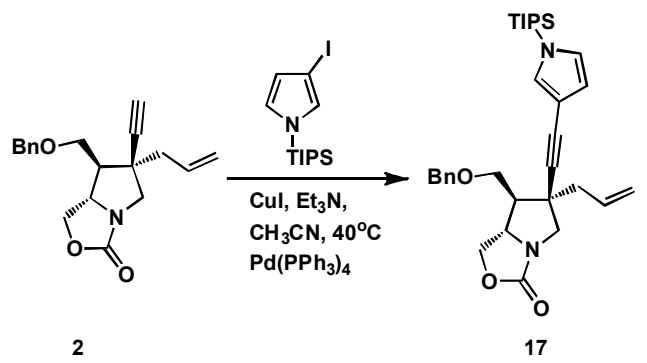

Experimental: To a solution of compound $2(400.0 \mathrm{mg}, 1.3 \mathrm{mmol})$ in dry acetonitrile $(50 \mathrm{~mL})$, anhydrous triethylamine $(1.8 \mathrm{~mL}, 12.8$ mmol, 10 equiv), $\mathrm{CuI}\left(12 \mathrm{mg}, 0.06 \mathrm{mmol}, 5 \%\right.$ equiv), $\mathrm{Pd}\left(\mathrm{PPh}_{3}\right)_{4}(74.0 \mathrm{mg}, 0.06 \mathrm{mmol}, 5 \%$ equiv) and 3-iodo-1-(triisopropylsilyl-1Hpyrrole (492.0 mg, $1.4 \mathrm{mmol}, 1.1$ equiv.) was added. The resulting mixture was stirred at $40{ }^{\circ} \mathrm{C}$ for $1 \mathrm{~h}$; TLC analysis indicated the consumption of starting material. The solvent was removed in vacuum and the resulting crude residue was purified via flash chromatography on silica gel (7:1 Hexanes:EtOAc) to give Compound $17(500.0 \mathrm{mg}, 73.1 \%)$ as a clear, colorless thick oil.

$\mathbf{R}_{\mathbf{f}}=\left(4: 1\right.$ Hexanes:EtOAc) 0.53. ${ }^{1} \mathbf{H}$ NMR: $\left(400 \mathrm{MHz}, \mathrm{CDCl}_{3}\right) \delta 7.23-7.32(\mathrm{~m}, 5 \mathrm{H}), 6.85(\mathrm{~s}, 1 \mathrm{H}), 6.64(\mathrm{~s}, 1 \mathrm{H}), 6.27(\mathrm{~s}, 1 \mathrm{H}), 5.87-5.98(\mathrm{~m}$, $1 \mathrm{H}), 5.11-5.17(\mathrm{t}, J=9.2 \mathrm{~Hz}, 2 \mathrm{H}), 4.46-4.51(\mathrm{~m}, 3 \mathrm{H}), 4.36-4.39(\mathrm{dd}, J=9.0,3.1 \mathrm{~Hz}, 1 \mathrm{H}), 4.04-4.09(\mathrm{~m}, 1 \mathrm{H}), 3.79-3.83(\mathrm{~d}, J=9.4,4.7 \mathrm{~Hz}$, $1 \mathrm{H}), 3.68-3.74(\mathrm{t}, J=10.1 \mathrm{~Hz}, 2 \mathrm{H}), 3.45-3.48(\mathrm{~d}, J=11.4 \mathrm{~Hz}, 1 \mathrm{H}), 2.46-2.51(\mathrm{dd}, J=13.5,6.6 \mathrm{~Hz}, 1 \mathrm{H}), 2.29-2.34(\mathrm{dd}, J=13.4,7.7 \mathrm{~Hz}$, $1 \mathrm{H}), 1.97-2.03(\mathrm{~m}, 1 \mathrm{H}), 1.37-1.44(\mathrm{~m}, 3 \mathrm{H}), 1.06-1.08 \mathrm{ppm}(\mathrm{d}, J=7.44 \mathrm{~Hz}, 18 \mathrm{H}) .{ }^{13} \mathbf{C} \mathbf{~ N M R}:\left(100 \mathrm{MHz}, \mathrm{CDCl}_{3}\right) \delta 161.2,137.9,133.5$, 128.3, 128.3, 127.9, 127.2, 127.2, 124.1, 118.8, 118.8, 113.6, 105.5, 87.7, 81.1, 73.3, 69.9, 67.7, 63.8, 58.3, 52.1, 46.5, 44.2, 17.6, 17.6, 17.6, 17.6, 17.6, 17.6, 11.7, 11.7, 11.7 ppm. IR $\left(v_{\max }\right): 2951,2867,1761,1633,1469,1389,1099 \mathrm{~cm}^{-1}$. MS: HRMS (ESI) m/z: Calculated 
for $\mathrm{C}_{32} \mathrm{H}_{44} \mathrm{~N}_{2} \mathrm{NaO}_{3} \mathrm{Si}(\mathrm{M}+\mathrm{Na})^{+}$555.3013, found 555.3016. OR: $[\alpha]_{\mathrm{D}}{ }^{20}=\left(\mathrm{c} 0.67, \mathrm{CH}_{2} \mathrm{Cl}_{2}\right)+39.9^{\circ}$ Compound 18:

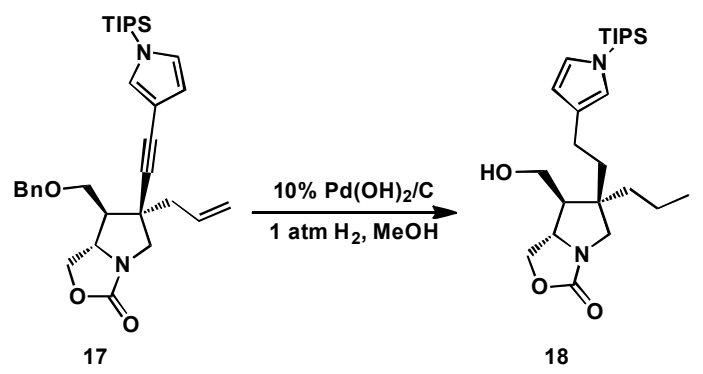

Experimental: Compound $17(450.0 \mathrm{mg}, 0.8 \mathrm{mmol})$ was dissolved in dry $\mathrm{MeOH}(300 \mathrm{~mL})$, followed by addition of $\mathrm{Pd}(\mathrm{OH})_{2} / \mathrm{C}(10 \%$, $45.0 \mathrm{mg})$. The reaction was placed under hydrogen atmosphere $(1 \mathrm{~atm})$ and was stirred at room temperature overnight. TLC analysis indicated the consumption of starting material. The reaction mixture was filtered over a Celite plug and washed with acetone. The filtrate was concentrated in vacuum. The crude organics was purified via flash chromatography on silica gel (2:1 Hexanes:EtOAc) to give 18 (297.0 $\mathrm{mg}, 78.3 \%$ ) as a clear, colorless thick oil.

$\mathbf{R}_{\mathbf{f}}=(1: 1$ Hexanes:EtOAc) 0.43 .

${ }^{1} \mathbf{H}$ NMR: $\left(400 \mathrm{MHz}, \mathrm{CDCl}_{3}\right) \delta 6.70(\mathrm{~s}, 1 \mathrm{H}), 6.51(\mathrm{~s}, 1 \mathrm{H}), 6.12(\mathrm{~s}, 1 \mathrm{H}), 4.51-4.56(\mathrm{t}, J=12.8 \mathrm{~Hz}, 1 \mathrm{H}), 4.34-4.37(\mathrm{dd}, J=13.6,5.9 \mathrm{~Hz}, 1 \mathrm{H})$, $3.96-4.01(\mathrm{td}, J=12.8,5.6 \mathrm{~Hz}, 1 \mathrm{H}), 3.90-3.92(\mathrm{dd}, J=15.3,6.5 \mathrm{~Hz}, 2 \mathrm{H}), 3.64-3.69(\mathrm{t}, J=15.2 \mathrm{~Hz}, 1 \mathrm{H}), 3.50-3.53(\mathrm{~d}, J=17.6 \mathrm{~Hz}, 1 \mathrm{H})$, $3.17-3.20(\mathrm{~d}, J=17.7 \mathrm{~Hz}, 1 \mathrm{H}), 2.37-2.46(\mathrm{~m}, 2 \mathrm{H}), 1.91-1.97(\mathrm{td}, J=14.6,6.5 \mathrm{~Hz}, 1 \mathrm{H}), 1.25-1.68(\mathrm{~m}, 10 \mathrm{H}), 1.08-1.10(\mathrm{~d}, J=11.1 \mathrm{~Hz}$, $18 \mathrm{H}), 0.90-0.94 \mathrm{ppm}(\mathrm{t}, J=10.5 \mathrm{~Hz}, 3 \mathrm{H})$.

${ }^{13}$ C NMR: $\left(100 \mathrm{MHz}_{\mathrm{CDCl}}\right) \delta 161.5,125.3,124.3,120.8,110.1,69.0,63.9,62.0,56.5,54.5,48.8,40.9,35.0,22.0,17.8,17.8,17.8$, $17.8,17.8,17.8,17.8,14.7,11.6,11.6,11.6 \mathrm{ppm}$.

IR $\left(v_{\max }\right): 3423,1632,1102 \mathrm{~cm}^{-1}$.

MS: HRMS (ESI) m/z: Calculated for $\mathrm{C}_{25} \mathrm{H}_{44} \mathrm{~N}_{2} \mathrm{NaO}_{3} \mathrm{Si}(\mathrm{M}+\mathrm{Na})^{+} 471.3013$, found 471.3011 .

OR: $[\alpha]_{\mathrm{D}}{ }^{20}=\left(\mathrm{c} 0.67, \mathrm{CH}_{2} \mathrm{Cl}_{2}\right)-10.5^{\circ}$.

Compound 19:

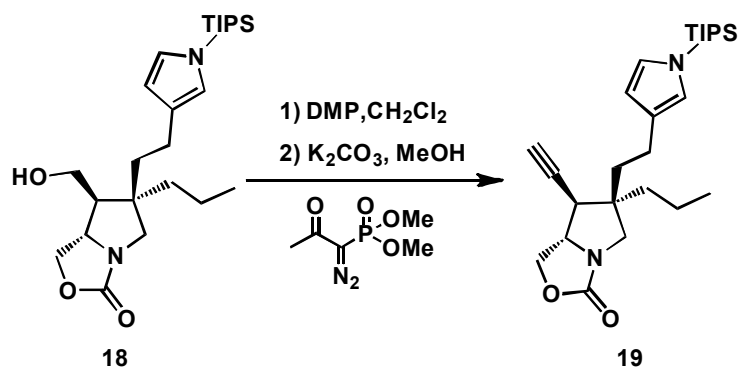

Experimental: Compound $18(250.0 \mathrm{mg}, 0.6 \mathrm{mmol})$ was dissolved in dry DCM $(30 \mathrm{~mL})$ and Dess-Martin periodinane (356.0 mg, 0.8 mmol, 1.5 equiv) was added. The reaction was allowed to stir at room temperature for 30 min until TLC indicated consumption of the starting material. The reaction was quenched with saturated aq. $\mathrm{Na}_{2} \mathrm{~S}_{2} \mathrm{O}_{3}$ and aq. $\mathrm{NaHCO}_{3}$. The aqueous layer was extracted with $\mathrm{CH}_{2} \mathrm{Cl}_{2}(2$ $\times 100 \mathrm{~mL}$ ), and the combined organic were washed with saturated aq. $\mathrm{Na}_{2} \mathrm{~S}_{2} \mathrm{O}_{3}$ and aq. $\mathrm{NaHCO}_{3}$, dried over $\mathrm{Na}_{2} \mathrm{SO}_{4}$, and concentrated in vacuum. The crude aldehyde was pure enough for further use. Under a nitrogen atmosphere, to a stirred solution of Ohira-Bestmann reagent $(430.0 \mathrm{mg}, 2.2 \mathrm{mmol}, 4.0$ equiv) in $\mathrm{MeOH}(15 \mathrm{~mL})$, anhydrous potassium carbonate $(232.0 \mathrm{mg}, 1.7 \mathrm{mmol}, 3.0$ equiv) was added. After stirring at $0{ }^{\circ} \mathrm{C}$ for 1.5 hours, the reaction was warmed to $20^{\circ} \mathrm{C}$ and a solution of above crude aldehyde in dry THF $(5 \mathrm{~mL})$ was added dropwisely. The mixture was stirred at $20^{\circ} \mathrm{C}$ for $1 \mathrm{~h}$ until TLC indicated consumption of the starting material. The mixture was filtered over a Celite plug, and the filter cake was washed with EtOAc. The combined organics was concentrated in vacuum and the residue was purified via flash chromatography on silica gel (7:1 Hexanes:EtOAc) to give $\mathbf{1 9}(177.0 \mathrm{mg}, 71.7 \%)$ as a clear, colorless thick oil.

$\mathbf{R}_{\mathbf{f}}=(4: 1$ Hexanes:EtOAc) 0.54 .

${ }^{1}$ H NMR: $\left(600 \mathrm{MHz}, \mathrm{CDCl}_{3}\right) \delta 6.71(\mathrm{~s}, 1 \mathrm{H}), 6.54(\mathrm{~s}, 1 \mathrm{H}), 6.17(\mathrm{~s}, 1 \mathrm{H}), 4.49-4.52(\mathrm{dd}, J=9.3,7.5 \mathrm{~Hz}, 1 \mathrm{H}), 4.27-4.29(\mathrm{dd}, J=9.4,2.6 \mathrm{~Hz}$, $1 \mathrm{H}), 3.99-4.00(\mathrm{~m}, 1 \mathrm{H}), 3.44-3.46(\mathrm{~d}, J=11.9 \mathrm{~Hz}, 1 \mathrm{H}), 3.28-3.30(\mathrm{~d}, J=10.7 \mathrm{~Hz}, 1 \mathrm{H}), 2.49-2.57(\mathrm{~m}, 2 \mathrm{H}), 2.45-2.47(\mathrm{dd}, J=9.9,2.5 \mathrm{~Hz}$, $1 \mathrm{H}), 2.23(\mathrm{~s}, 1 \mathrm{H}), 2.00-2.06(\mathrm{~m}, 1 \mathrm{H}), 1.73-1.78(\mathrm{~m}, 1 \mathrm{H}), 1.59-1.63(\mathrm{~m}, 2 \mathrm{H}), 1.38-1.44(\mathrm{~m}, 5 \mathrm{H}), 1.09-1.10(\mathrm{~d}, J=7.5 \mathrm{~Hz}, 18 \mathrm{H}), 0.90-0.94$ $\operatorname{ppm}(\mathrm{t}, J=10.5 \mathrm{~Hz}, 3 \mathrm{H})$.

${ }^{13}$ C NMR: $\left(150 \mathrm{MHz}, \mathrm{CDCl}_{3}\right) \delta 161.1,125.4,124.3,120.9,110.3,79.8,74.0,66.4,63.9,55.9,50.0,45.4,41.6,36.0,21.8,18.0,17.9,17.9$, 17.9, 17.9, 17.9, 17.9,14.6,11.7,11.7, $11.7 \mathrm{ppm}$.

MS: HRMS (ESI) m/z: Calculated for $\mathrm{C}_{26} \mathrm{H}_{42} \mathrm{~N}_{2} \mathrm{NaO}_{2} \mathrm{Si}(\mathrm{M}+\mathrm{Na})^{+} 465.2908$, found 465.2911.

IR $\left(v_{\max }\right): 1630,1388,1101,620 \mathrm{~cm}^{-1}$.

OR: $[\alpha]_{\mathrm{D}}^{20}=\left(\mathrm{c} 1.00, \mathrm{CH}_{2} \mathrm{Cl}_{2}\right)+28.0^{\circ}$ 
Compound 20:

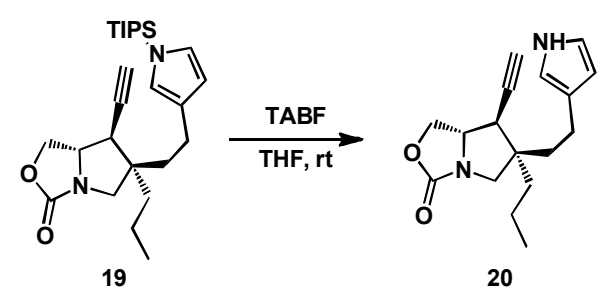

Experimental: Under nitrogen atmosphere, to a stirred solution of compound $19(0.1 \mathrm{~g}, 0.4 \mathrm{mmol})$ in dry THF (10 mL), tetra- $n$-butyl ammonium fluoride $(35.0 \mathrm{uL}$ of a $1 \mathrm{M}$ solution in THF, $0.04 \mathrm{mmol}, 0.1$ equiv) was added. Once the addition completed, the reaction was monitored by TLC. Upon completion (1 hour), the mixture was concentrated in vacuum and purified by preparative TLC (2:1 Hexanes:EtOAc) to give $\mathbf{2 0}(55.0 \mathrm{mg}, 84.6 \%)$ as a clear, colorless thick oil.

$\mathbf{R}_{\mathbf{f}}=\left(1: 1\right.$ Hexanes : EtOAc) 0.32. ${ }^{1} \mathbf{H}$ NMR: $\left(600 \mathrm{MHz}, \mathrm{CDCl}_{3}\right) \delta 8.10(\mathrm{br}, 1 \mathrm{H}), 6.75(\mathrm{~s}, 1 \mathrm{H}), 6.62(\mathrm{~s}, 1 \mathrm{H}), 6.12(\mathrm{~s}, 1 \mathrm{H}), 4.49-4.52(\mathrm{dd}, J=$ 9.2, 7.7Hz, 1H), 4.27-4.29 (dd, $J=9.3,2.4 \mathrm{~Hz}, 1 \mathrm{H}), 3.95-3.98(\mathrm{~m}, 2 \mathrm{H}), 3.45-3.47(\mathrm{~d}, J=11.9 \mathrm{~Hz}, 1 \mathrm{H}), 3.27-3.29(\mathrm{~d}, J=12.0 \mathrm{~Hz}, 1 \mathrm{H})$, 2.46-2.60 (m, 3H), $2.23(\mathrm{~s}, 1 \mathrm{H}), 2.02-2.07(\mathrm{dd}, J=13.2,5.3 \mathrm{~Hz}, 1 \mathrm{H}), 1.73-1.81(\mathrm{~m}, 1 \mathrm{H}), 1.31-1.42(\mathrm{~m}, 3 \mathrm{H}), 0.90-0.93 \mathrm{ppm}(\mathrm{t}, J=10.5 \mathrm{~Hz}$, $3 \mathrm{H}) .{ }^{13} \mathrm{C}$ NMR: $\left(150 \mathrm{MHz}, \mathrm{CDCl}_{3}\right) \delta 161.1,123.6,117.9,114.8,108.3,79.7,74.1,65.4,63.9,55.9,50.0,45.4,41.5,36.3,21.6,18.0,14.6$ ppm. IR $\left(v_{\max }\right): 2923,1636,1386,1103 \mathrm{~cm}^{-1}$. MS: HRMS (ESI) m/z: Calculated for $\mathrm{C}_{17} \mathrm{H}_{22} \mathrm{~N}_{2} \mathrm{NaO}_{2}(\mathrm{M}+\mathrm{Na})^{+} 309.1573$, found 309.1579. OR: $[\alpha]_{D}{ }^{20}=\left(\mathrm{c} 0.50, \mathrm{CH}_{2} \mathrm{Cl}_{2}\right)+42.0^{\circ}$.

Compound 1:

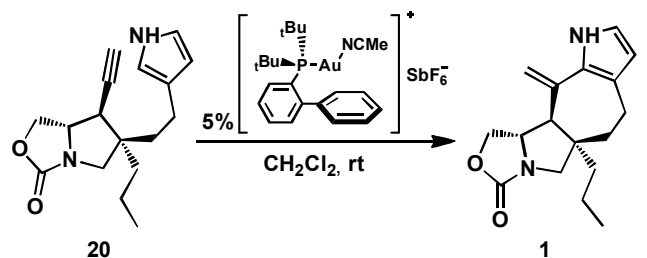

Experimental: In the nitrogen-filled glove box, alkyne $20(20.0 \mathrm{mg}, 0.07 \mathrm{mmol})$ was dissolved in dry DCM (15 $\mathrm{mL})$, then the JohnPho$\mathrm{sAu}(\mathrm{NCMe})] \mathrm{SbF}_{6}(3 \mathrm{mg}, 0.004 \mathrm{mmol}, 5 \%$ equiv) was added. The reaction was allowed to stir at room temperature for $30 \mathrm{~min}$. Once the color of the reaction turn to pink, the reaction was checked by TLC and starting material was found to be completely consumed. The mixture was concentrated in vacuum and purified by preparative TLC (2:1 EtOAc:Hexanes) to give Compound 1 (16.7 mg, 81.2\%) as a white solid.

$\mathbf{R}_{\mathbf{f}}=\left(1\right.$ : 1 Hexanes : EtOAc) 0.45. ${ }^{1} \mathbf{H}$ NMR: $\left(600 \mathrm{MHz}, \mathrm{CDCl}_{3}\right) \delta 8.00(\mathrm{br}, 1 \mathrm{H}), 6.67(\mathrm{~s}, 1 \mathrm{H}), 6.01(\mathrm{~s}, 1 \mathrm{H}), 5.04(\mathrm{~s}, 1 \mathrm{H}), 4.94(\mathrm{~m}, 1 \mathrm{H})$, 4.25-4.28 (dd, $J=8.9,7.5 \mathrm{~Hz}, 1 \mathrm{H}), 4.02-4.04(\mathrm{dd}, J=9.0,2.2 \mathrm{~Hz}, 1 \mathrm{H}), 3.88(\mathrm{~m}, 1 \mathrm{H}), 3.73-3.75(\mathrm{~d}, J=12.2 \mathrm{~Hz}, 1 \mathrm{H}), 3.20-3.22(\mathrm{~d}, J=12.2$ $\mathrm{Hz}, 1 \mathrm{H}), 2.71-2.73(\mathrm{~m}, 1 \mathrm{H}), 2.62-2.67(\mathrm{~m}, 1 \mathrm{H}), 2.36-2.38(\mathrm{~d}, J=10.6 \mathrm{~Hz}, 1 \mathrm{H}), 1.80-1.84(\mathrm{dd}, J=14.3,6.8 \mathrm{~Hz}, 1 \mathrm{H}), 1.66-1.68(\mathrm{~m}, 1 \mathrm{H})$, 1.31-1.38 (m, 2H), 1.24-1.29 (m, 2H), 0.90-0.94 ppm (t, $J=14.0 \mathrm{~Hz}, 3 \mathrm{H}) .{ }^{13} \mathbf{C}$ NMR: $\left(150 \mathrm{MHz}, \mathrm{CDCl}_{3}\right) \delta 161.8,136.8,126.5,123.3$, 118.0, 110.6, 110.4, 66.4, 63.3, 62.9, 55.8, 50.8, 44.9, 37.7, 22.7, 18.4, $14.7 \mathrm{ppm}$. IR ( $\left.v_{\max }\right): 2925,1633,1102 \mathrm{~cm}^{-1}$. MS: HRMS (ESI) m/z: Calculated for $\mathrm{C}_{17} \mathrm{H}_{22} \mathrm{~N}_{2} \mathrm{NaO}_{2}(\mathrm{M}+\mathrm{Na})^{+} 309.1573$, found 309.1581. MP: $191-193{ }^{\circ} \mathrm{C}$. OR: $[\alpha]_{\mathrm{D}}{ }^{20}=\left(\mathrm{c} 0.50, \mathrm{CH}_{2} \mathrm{Cl}_{2}\right)-56.0^{\circ}$. 


\section{Compound $3{ }^{1}$ H NMR}

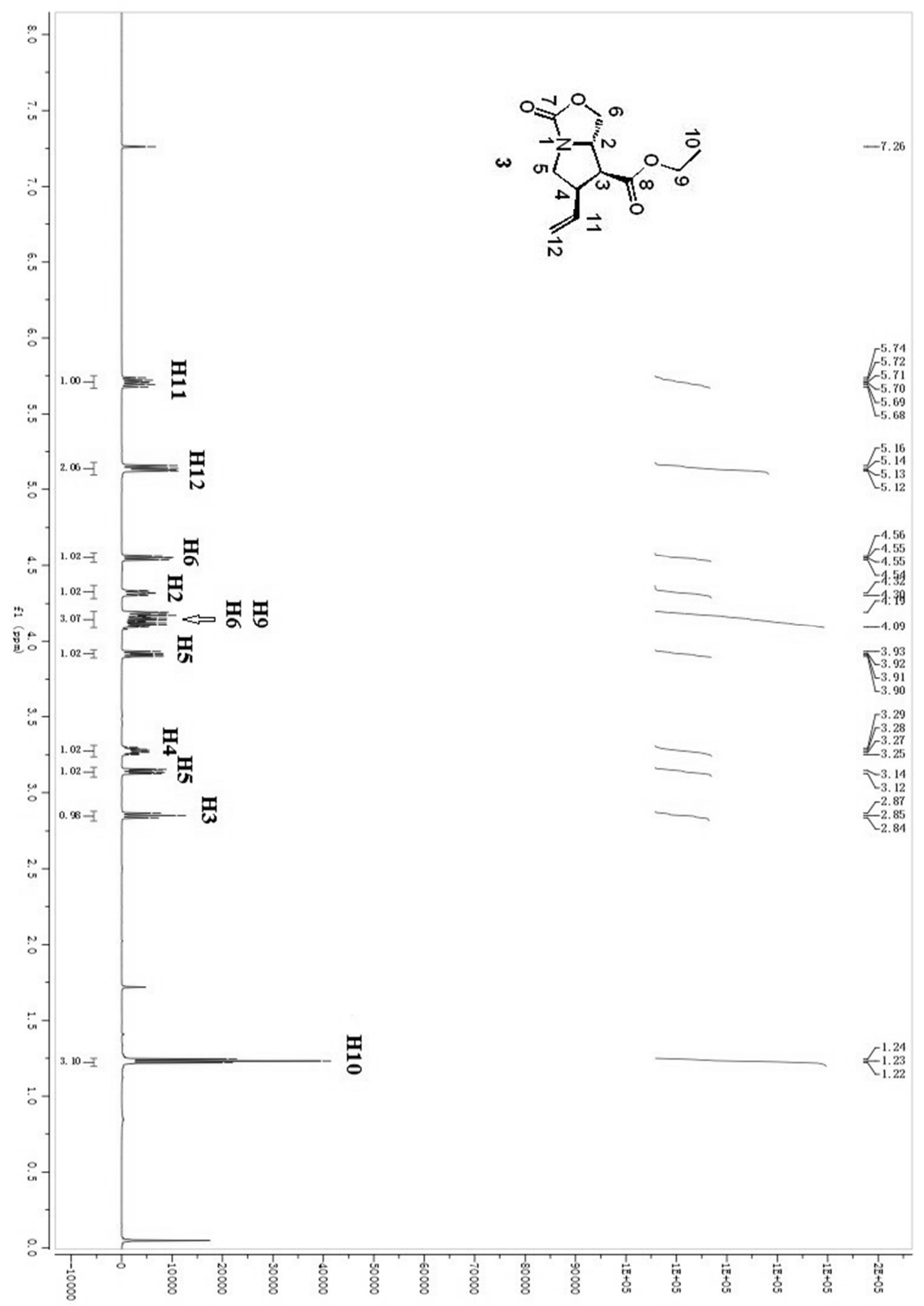


Compound $3{ }^{13} \mathrm{C}$ NMR

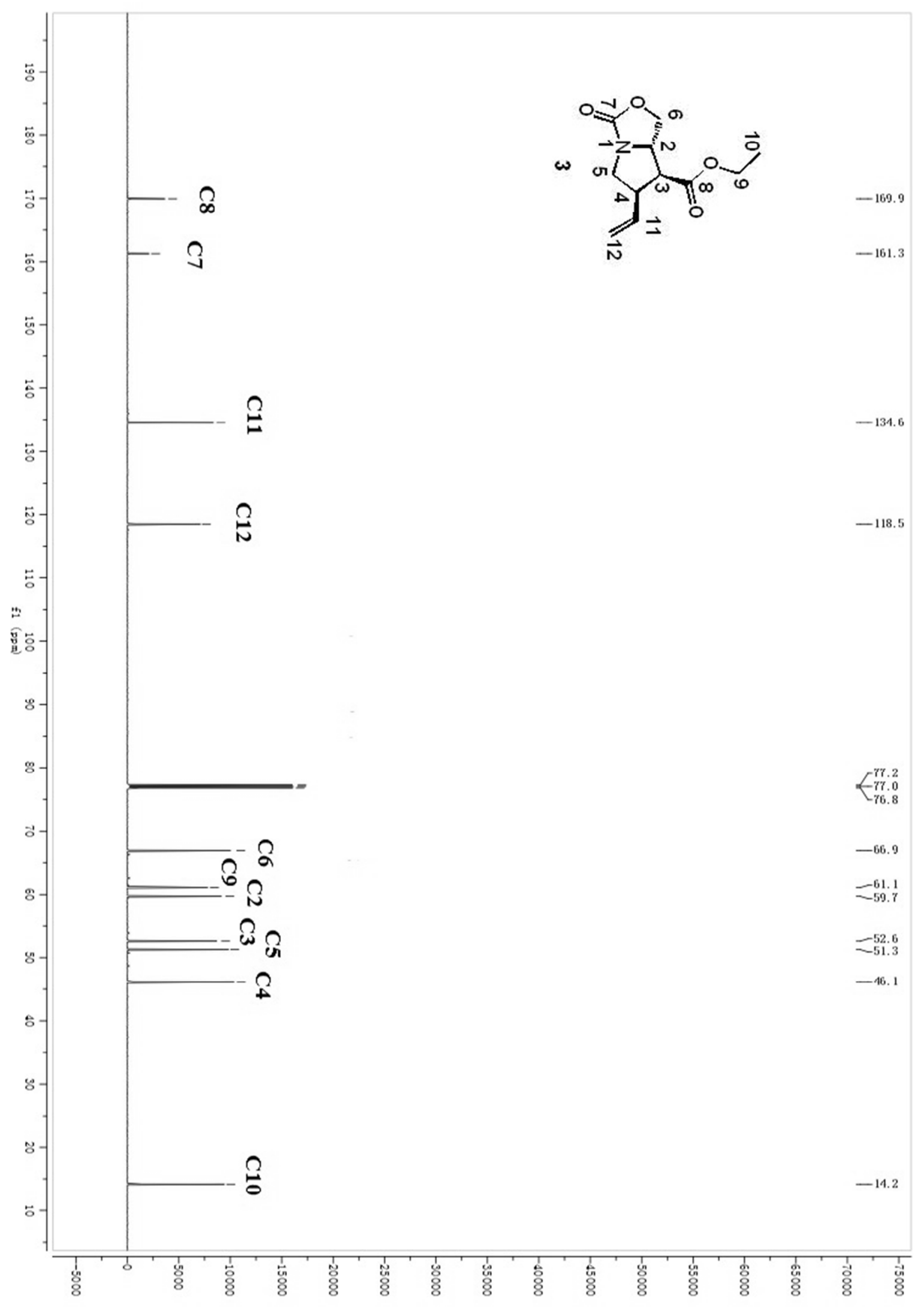




\section{Compound 3 DEPT}

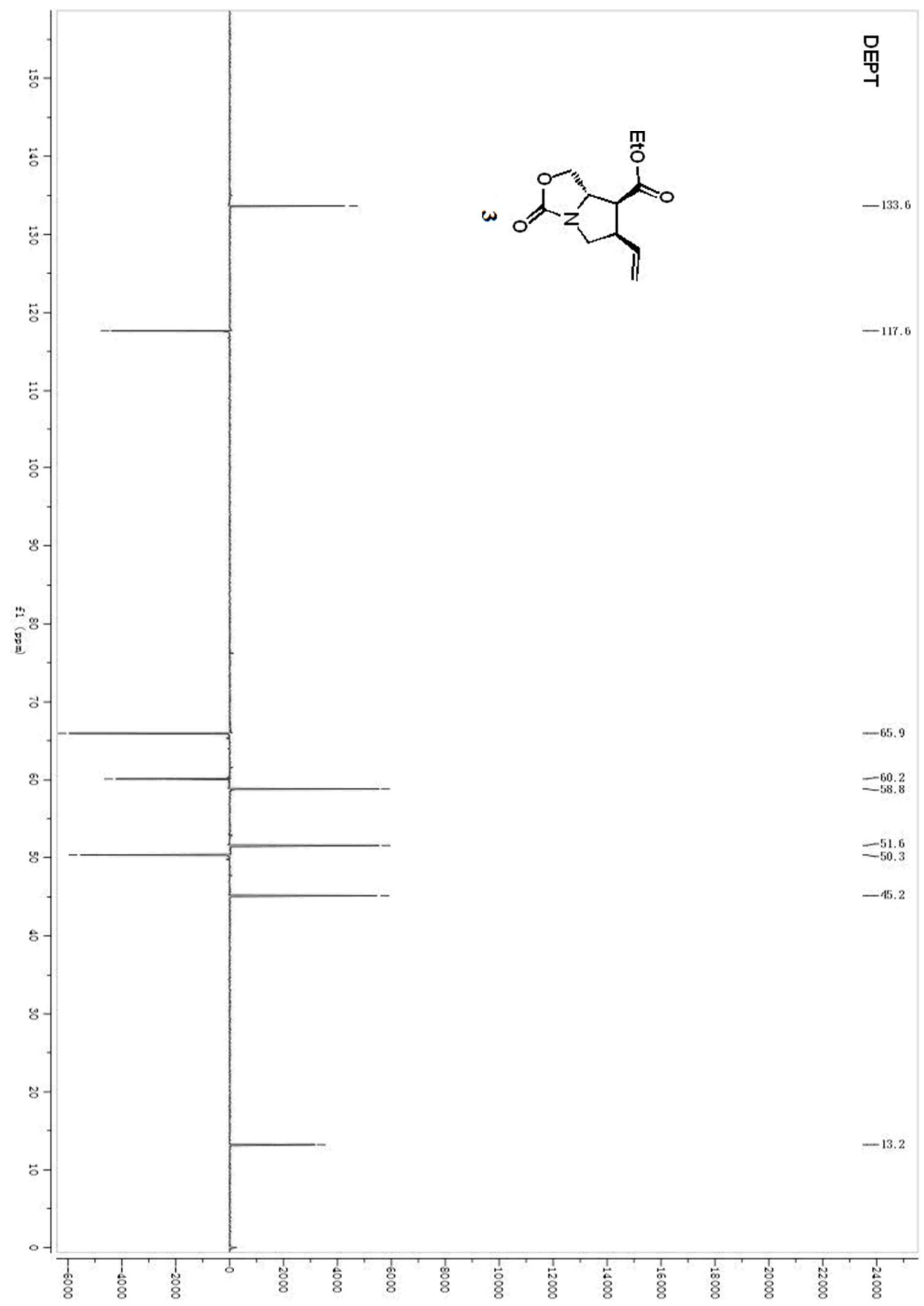

S-10 
Compound 3 HMQC

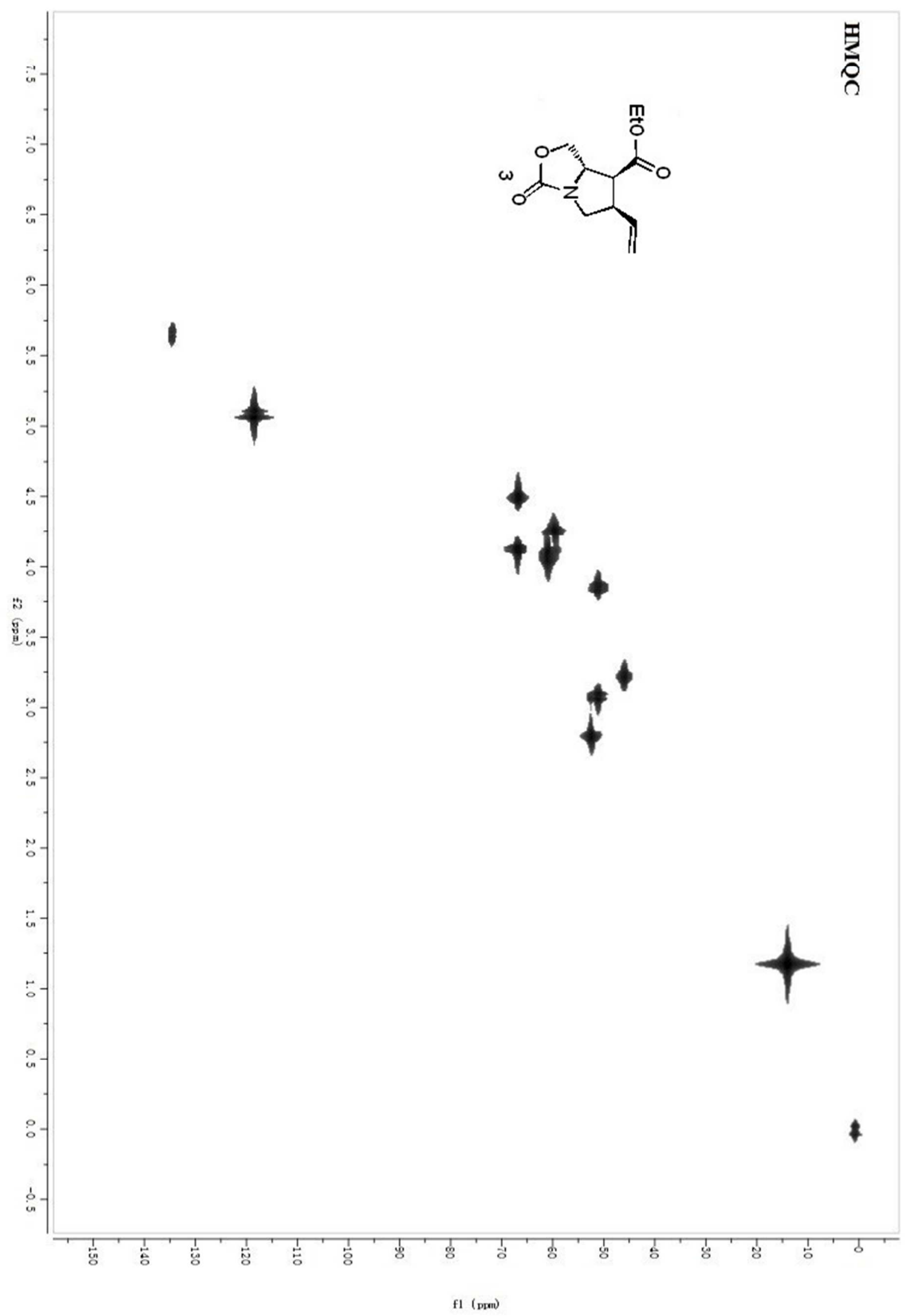

S-11 
Compound 3 NOEDS
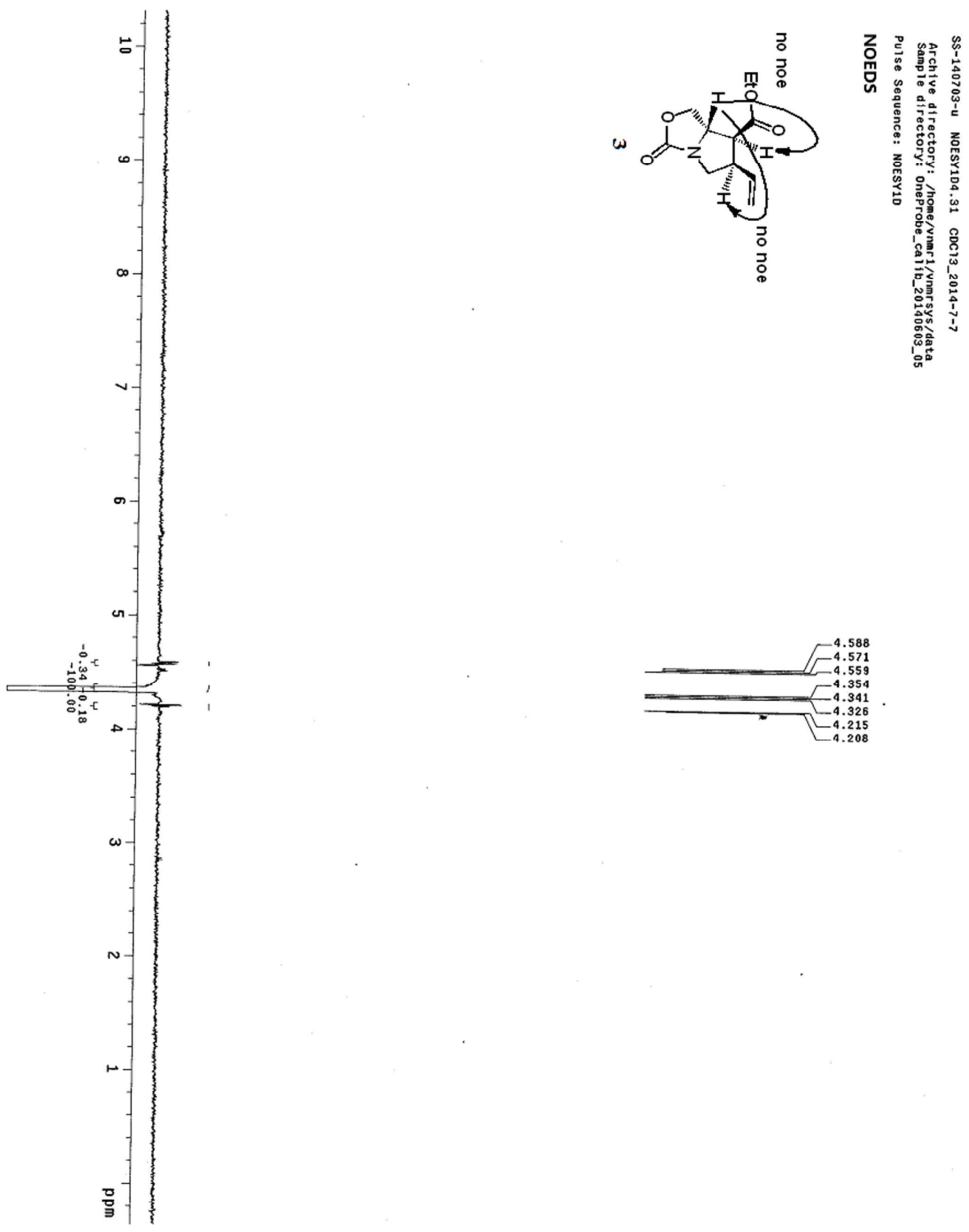


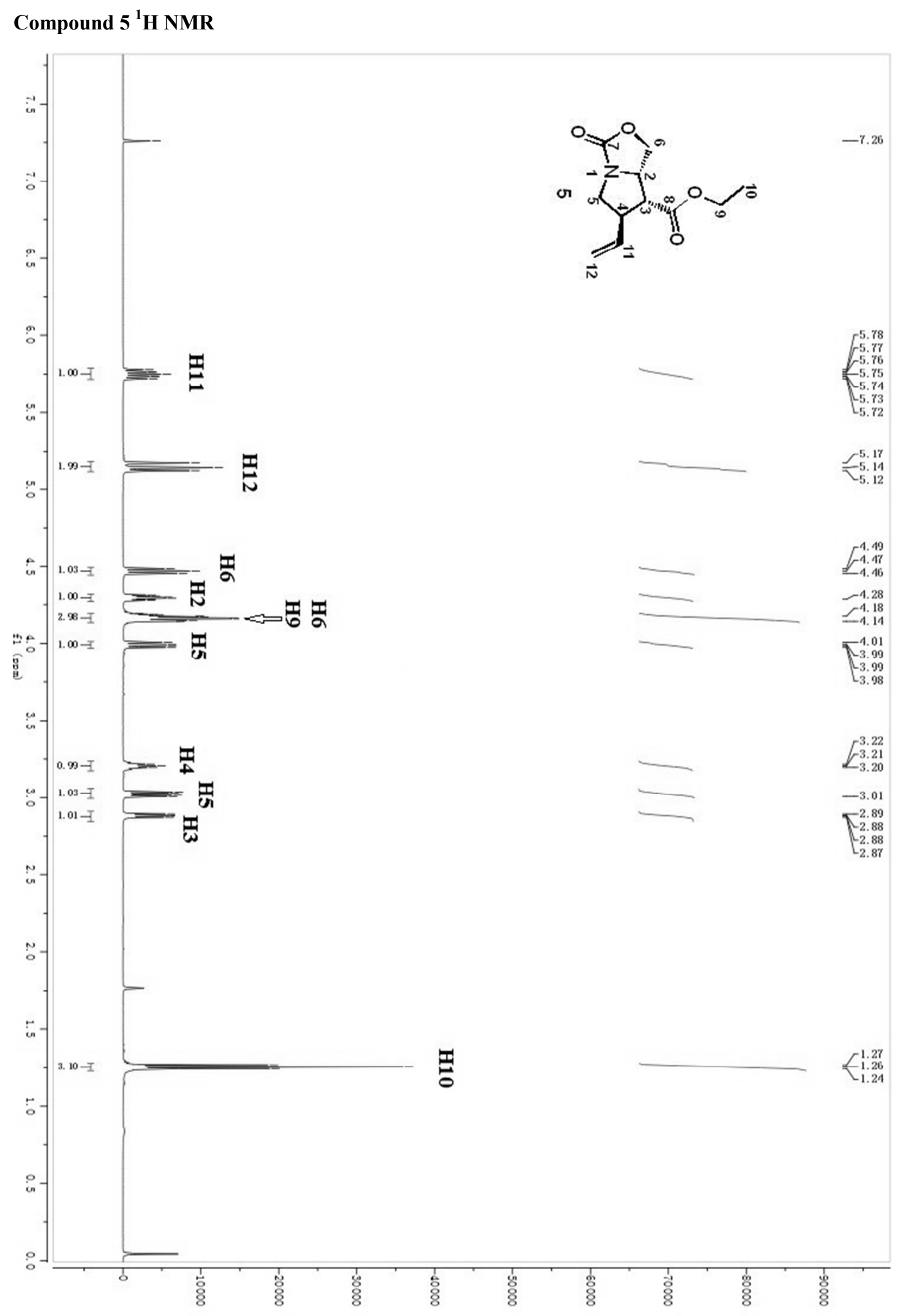

S-13 
Compound $5{ }^{13} \mathrm{C}$ NMR

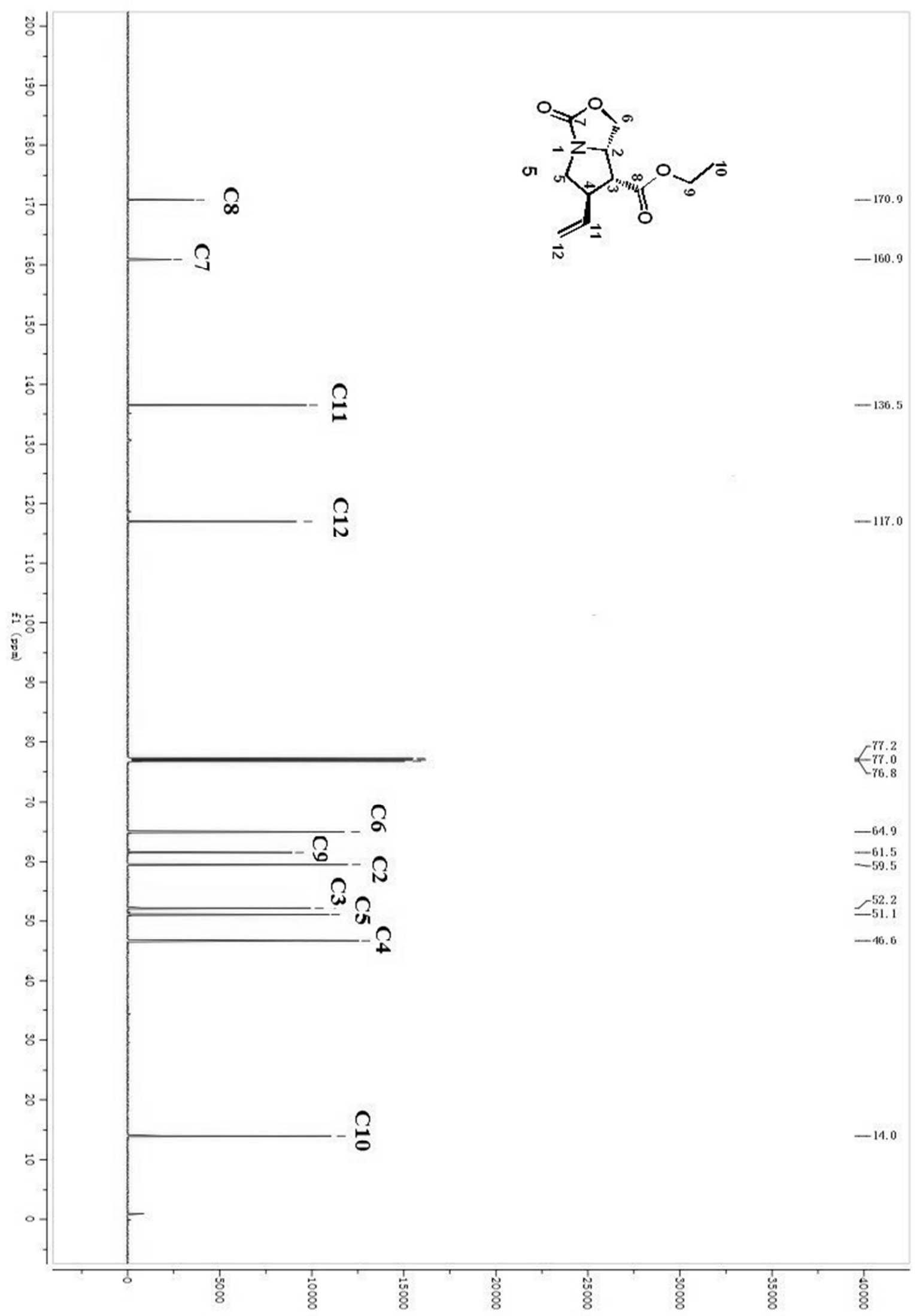




\section{Compound 5 DEPT}

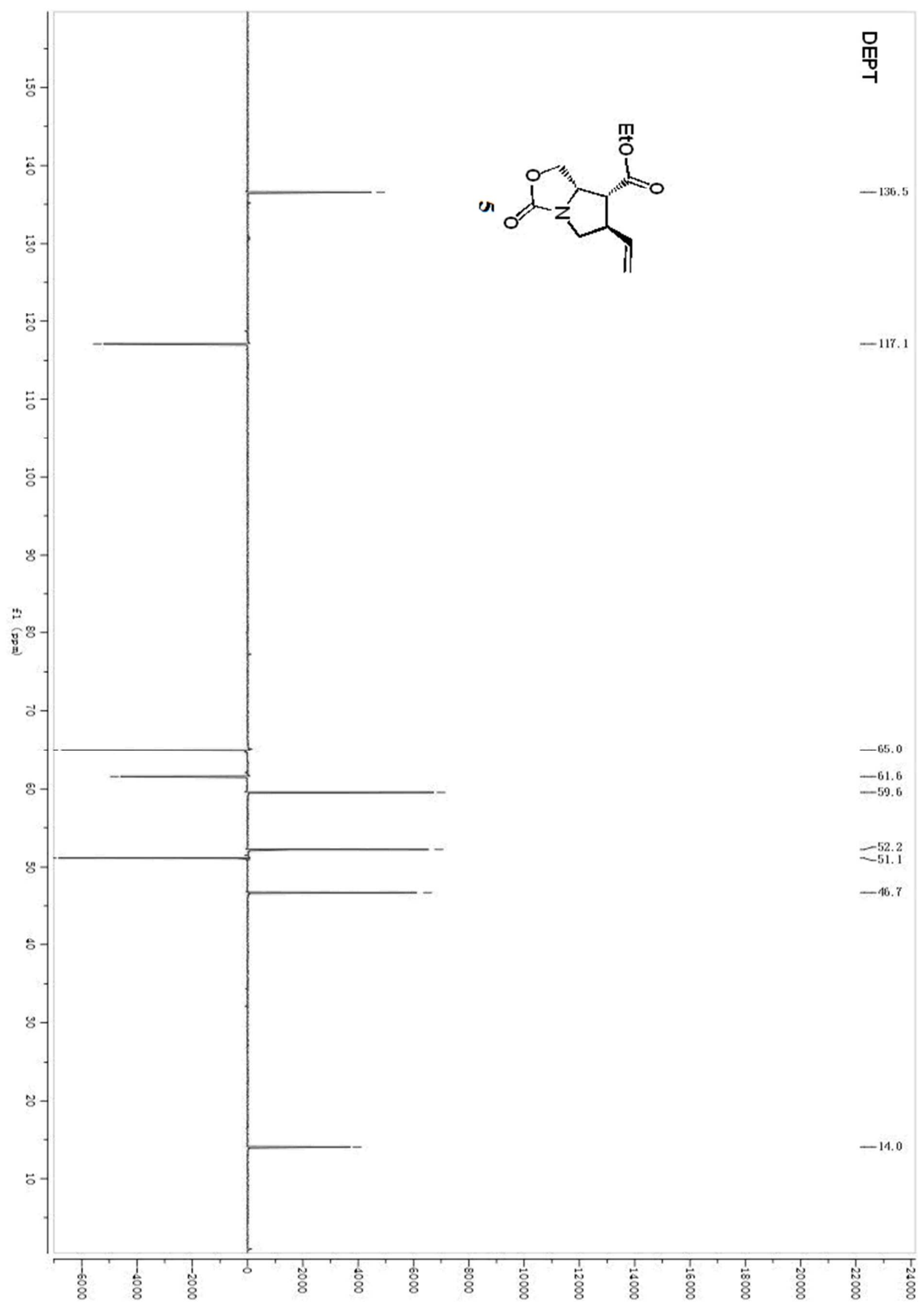

S-15 


\section{Compound 5 HMQC}

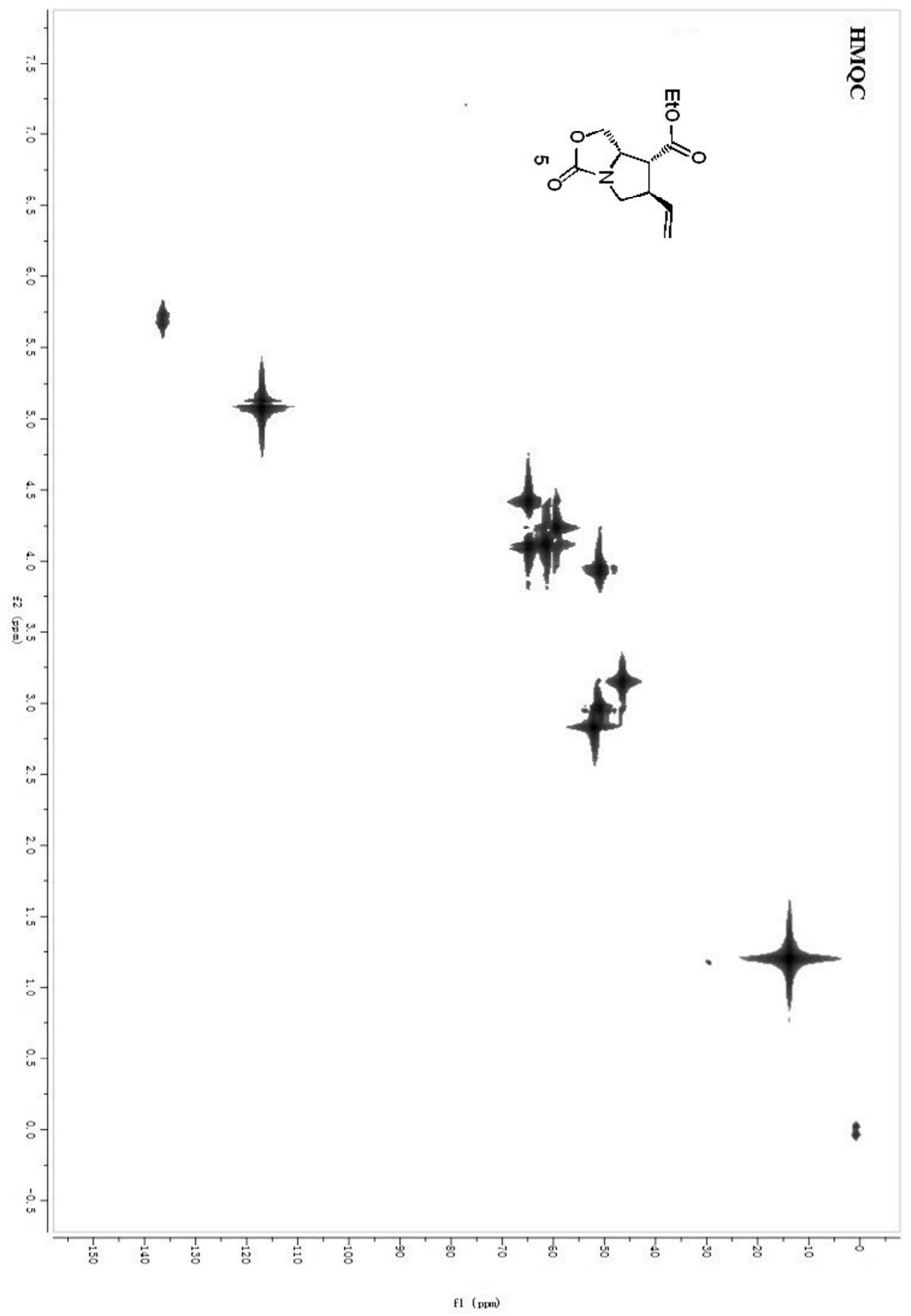


Compound 5 NOEDS
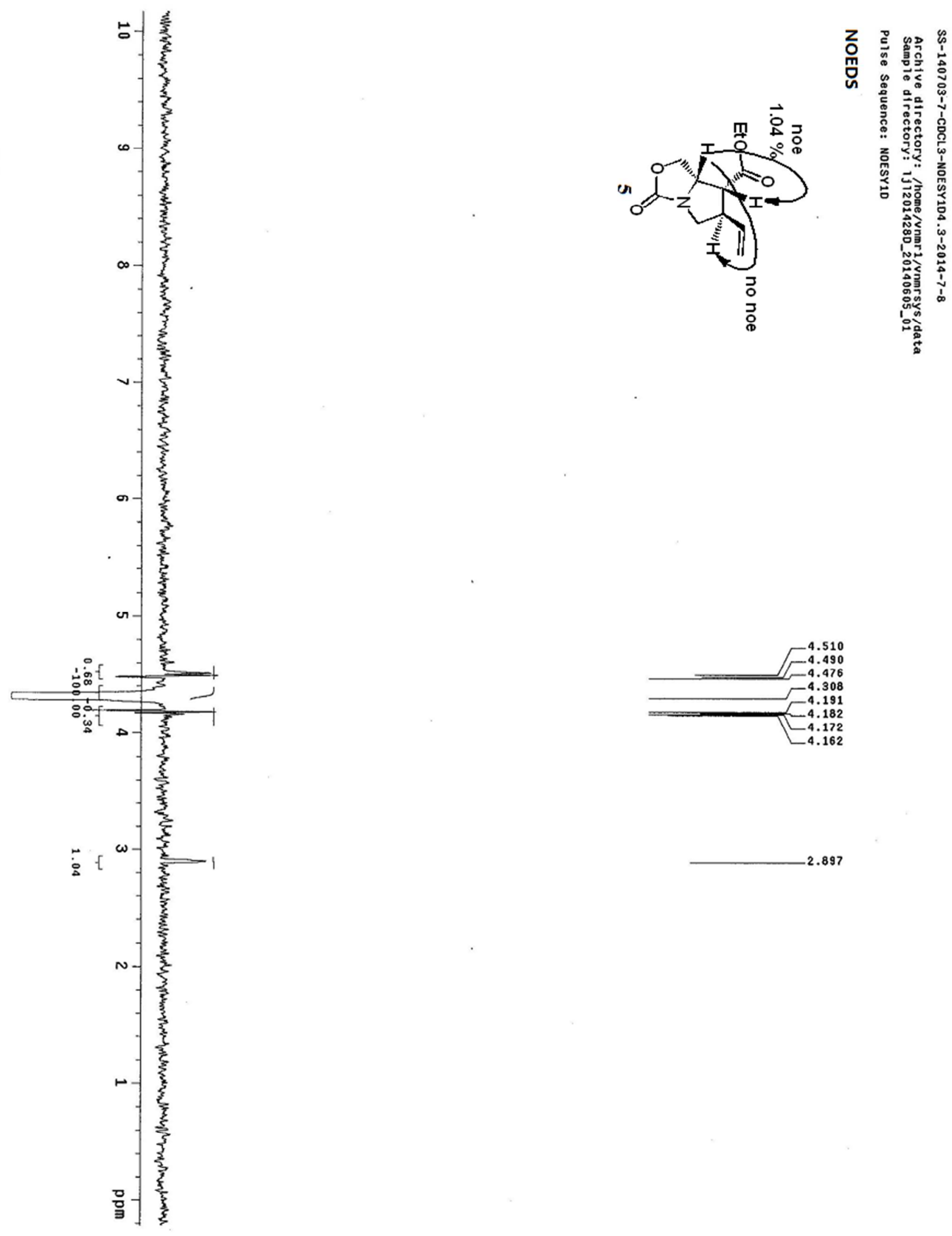


\section{Compound 3a ${ }^{1} \mathrm{H}$ NMR}

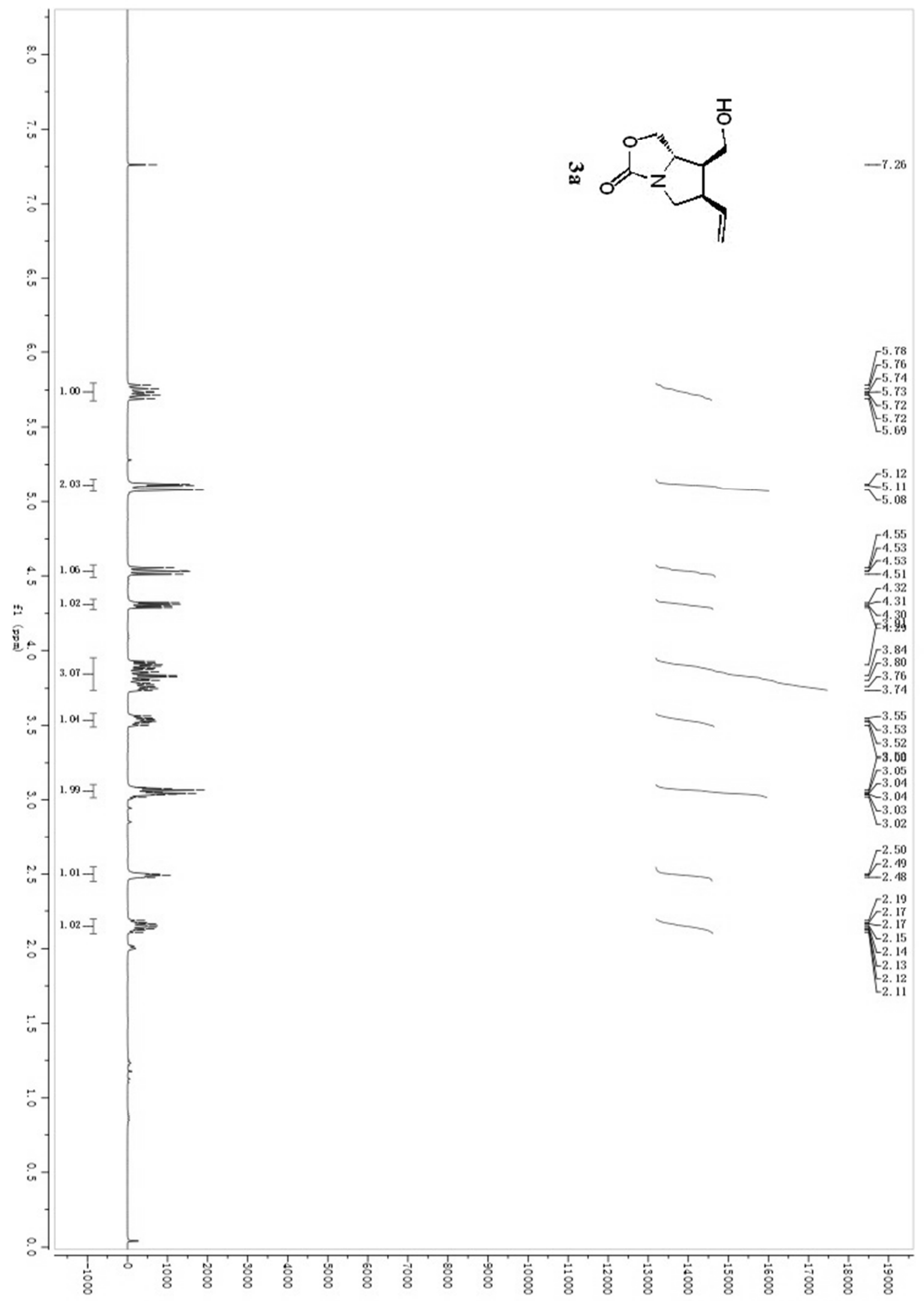




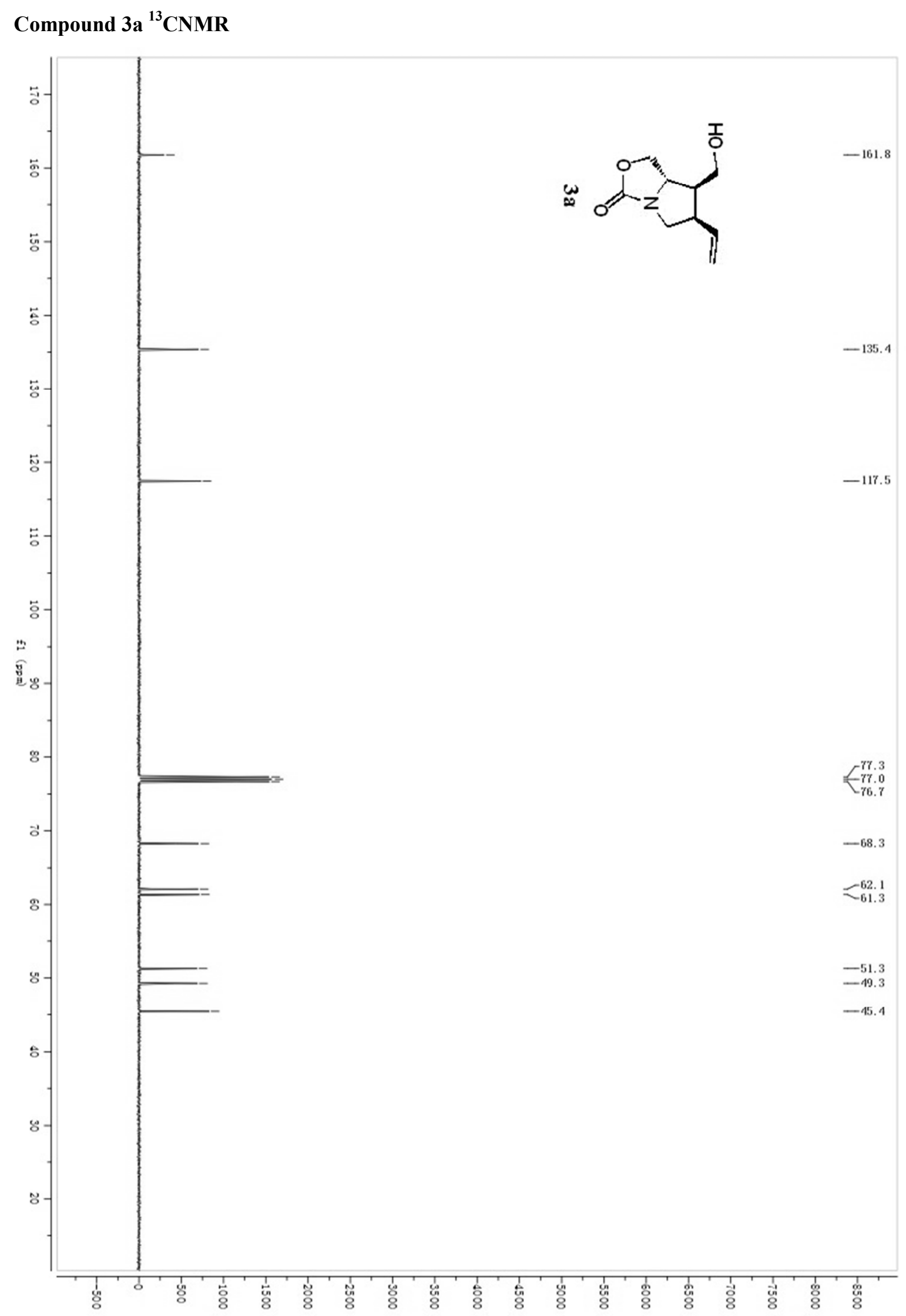




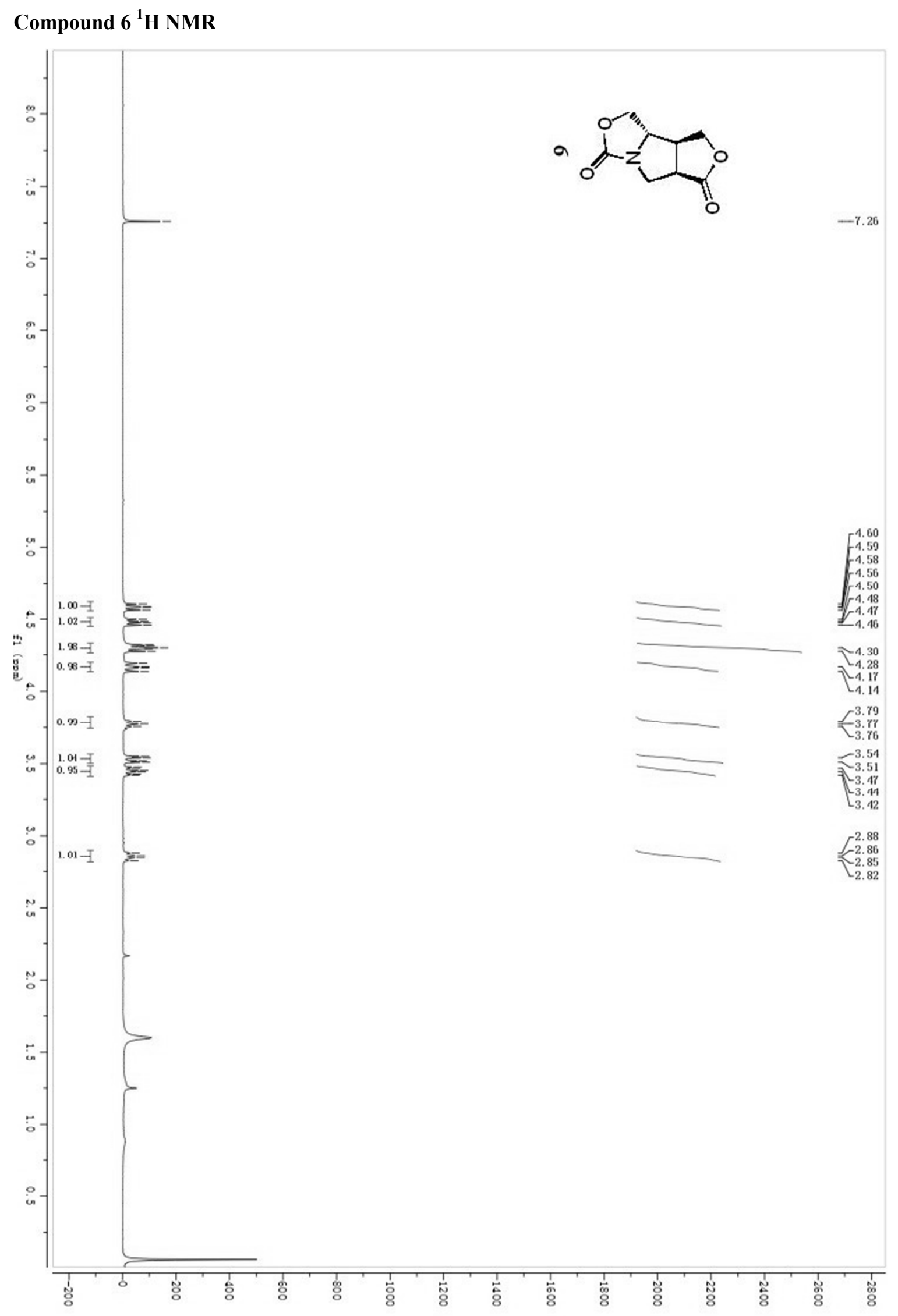




$$
[
$$




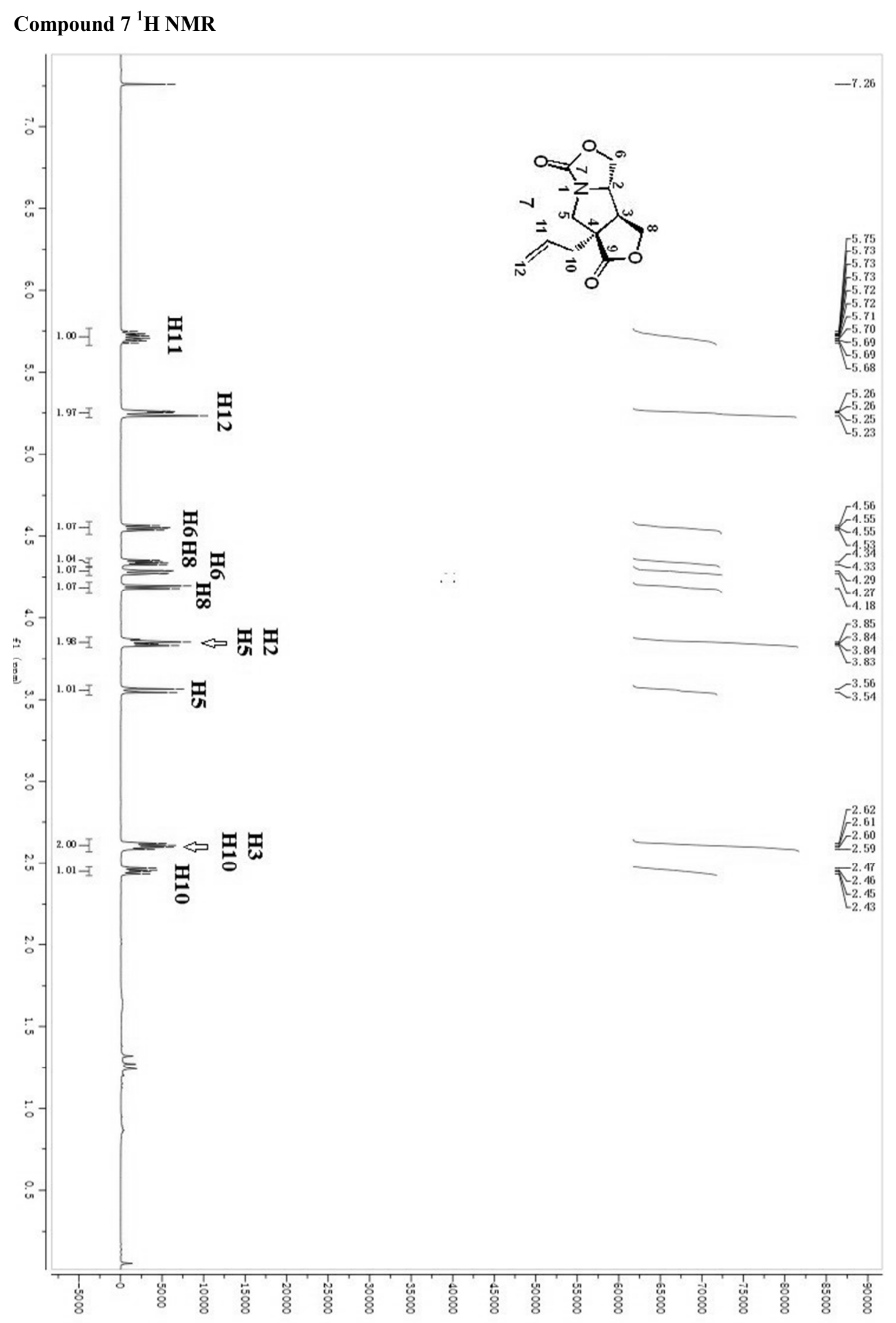


Compound $7{ }^{13} \mathrm{C}$ NMR

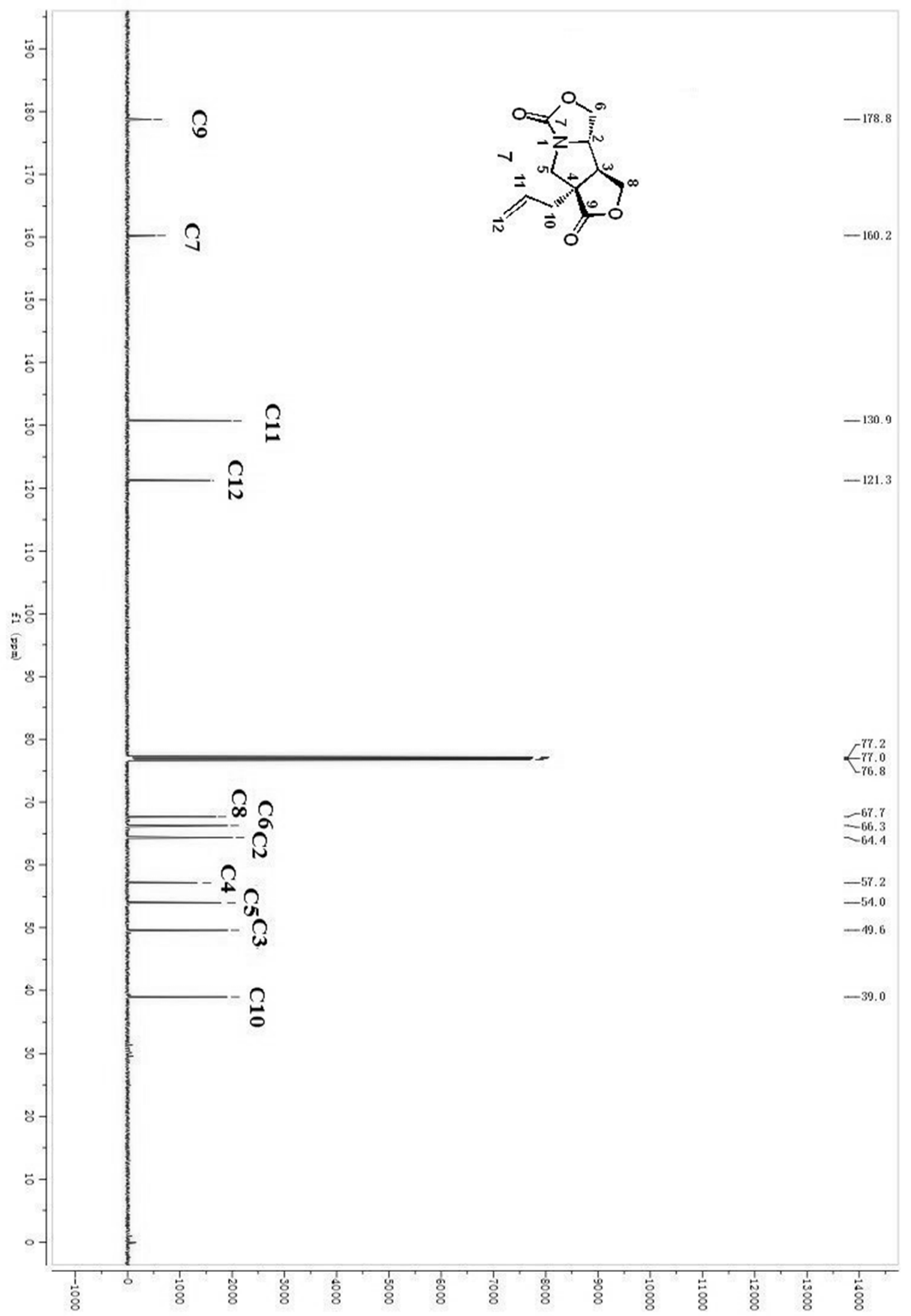




\section{Compound 7 DEPT}

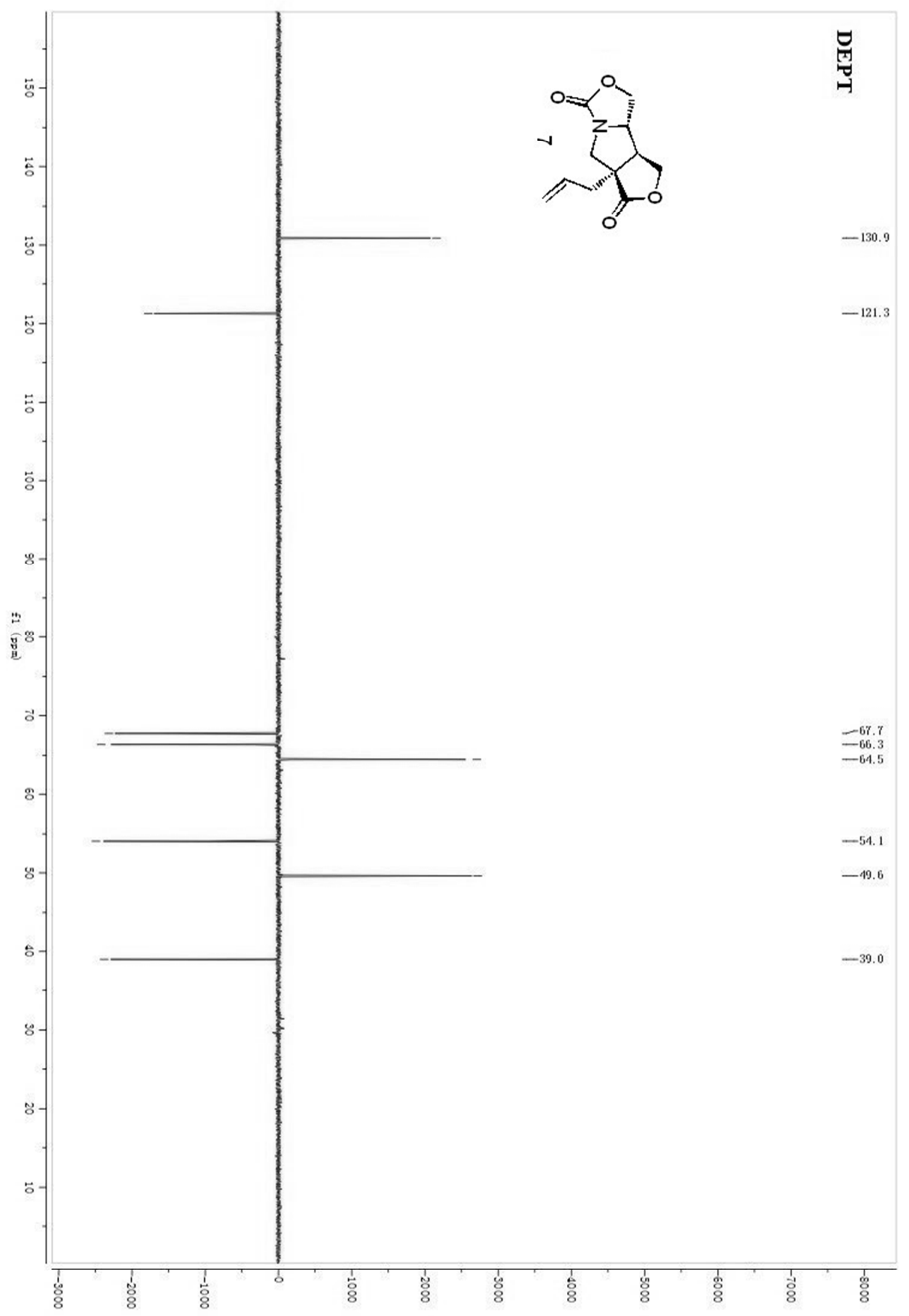




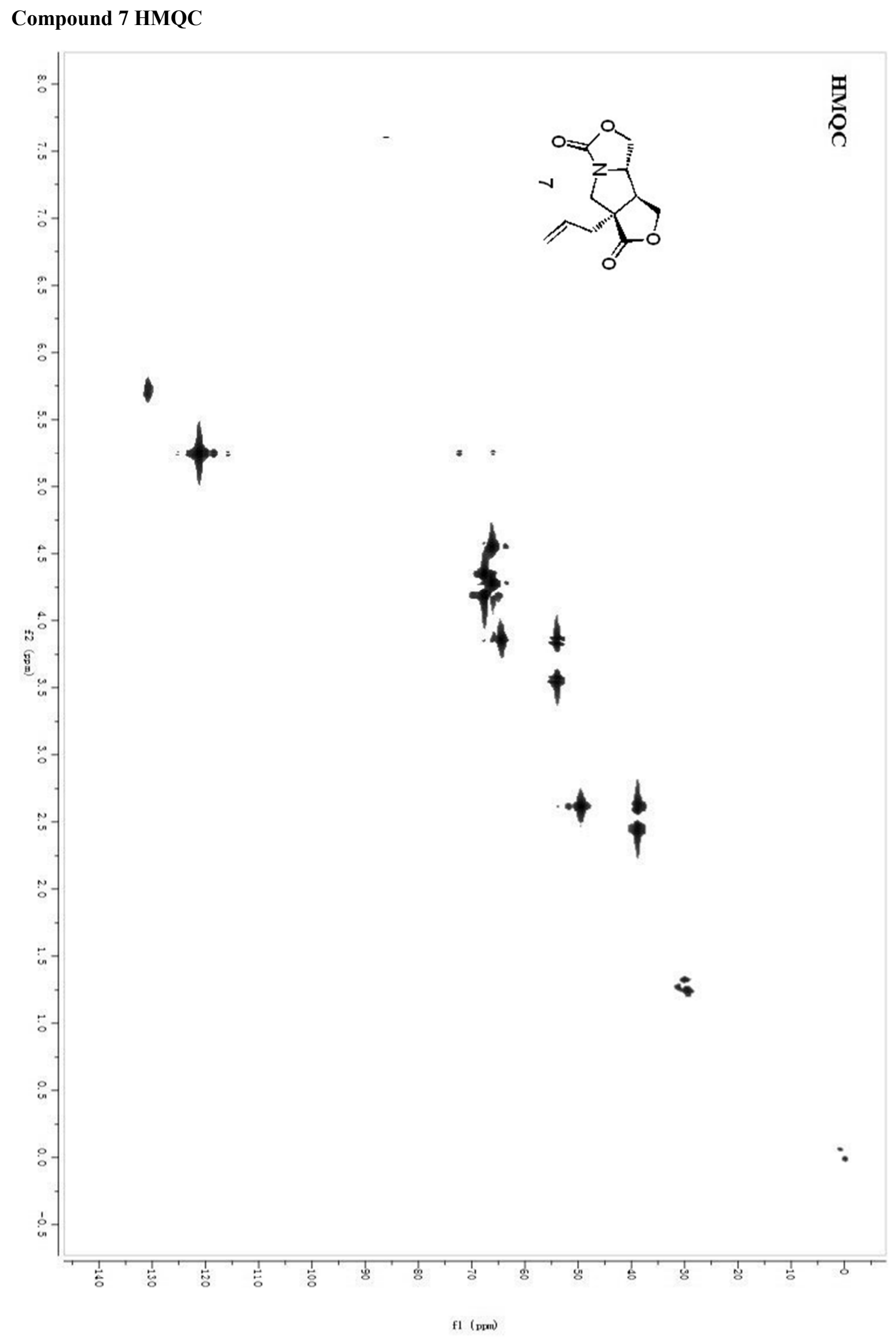




\section{Compound $8{ }^{1}$ H NMR}

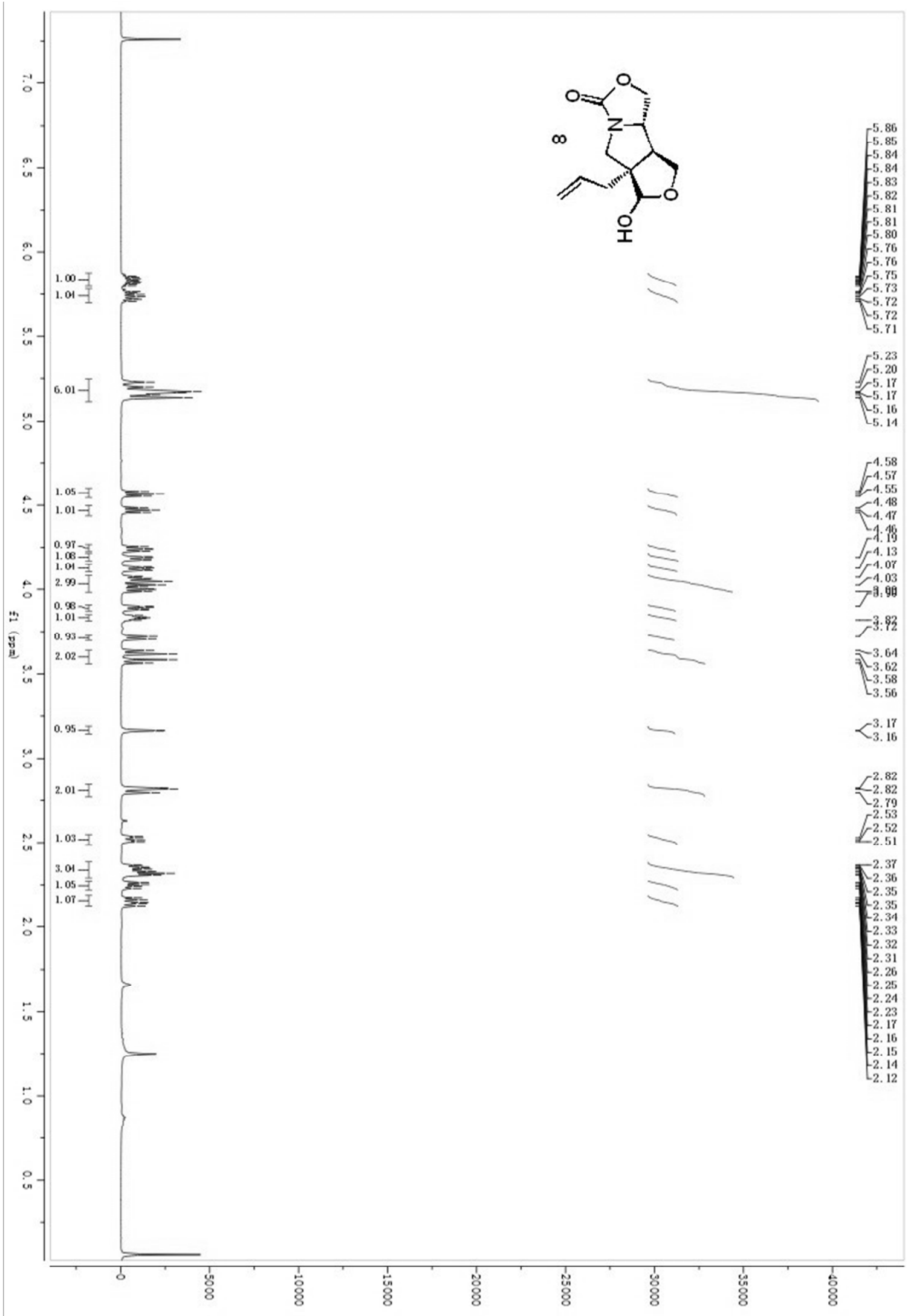


Compound $8{ }^{13} \mathrm{C}$ NMR

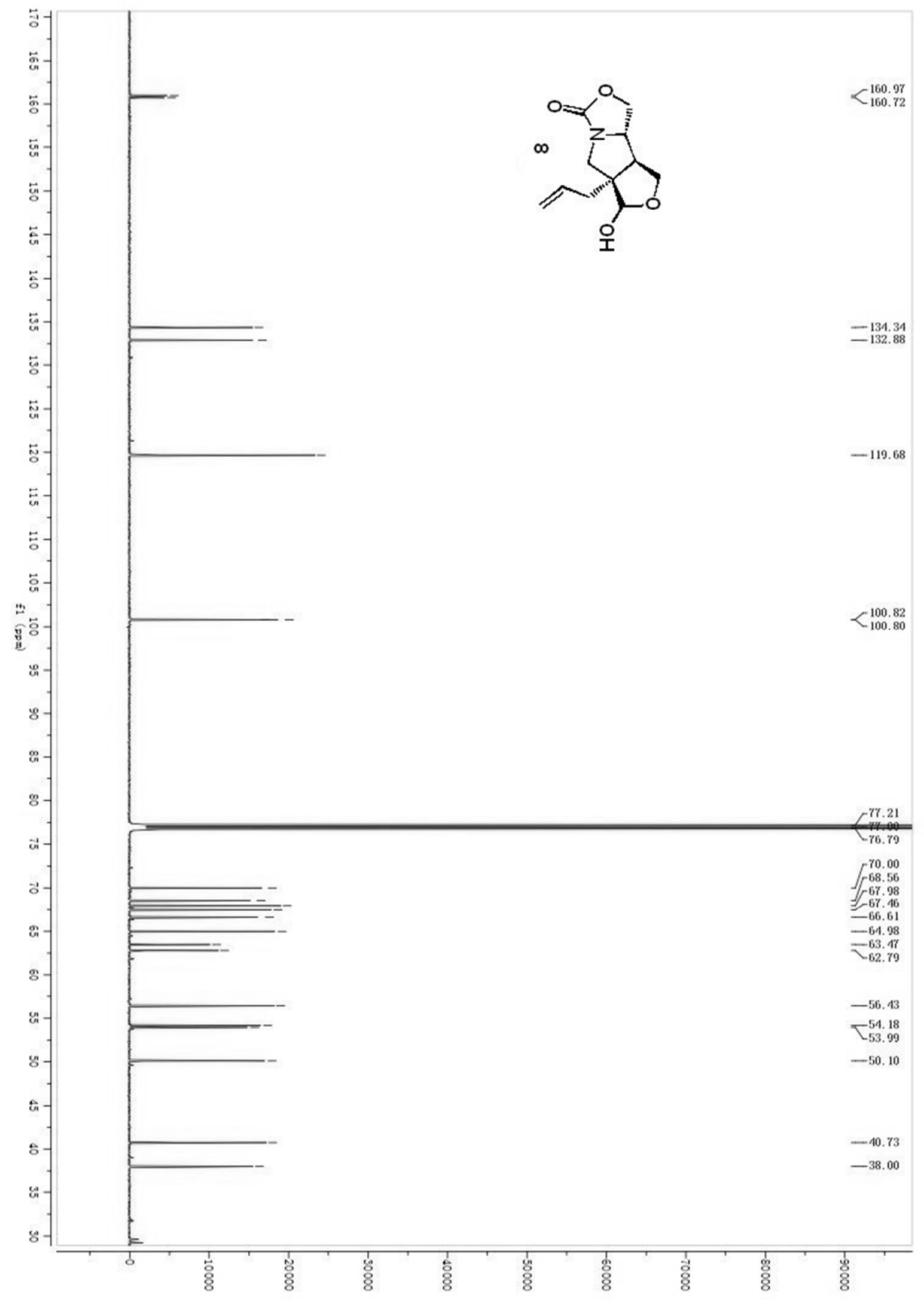




\section{Compound $10{ }^{1}$ H NMR}

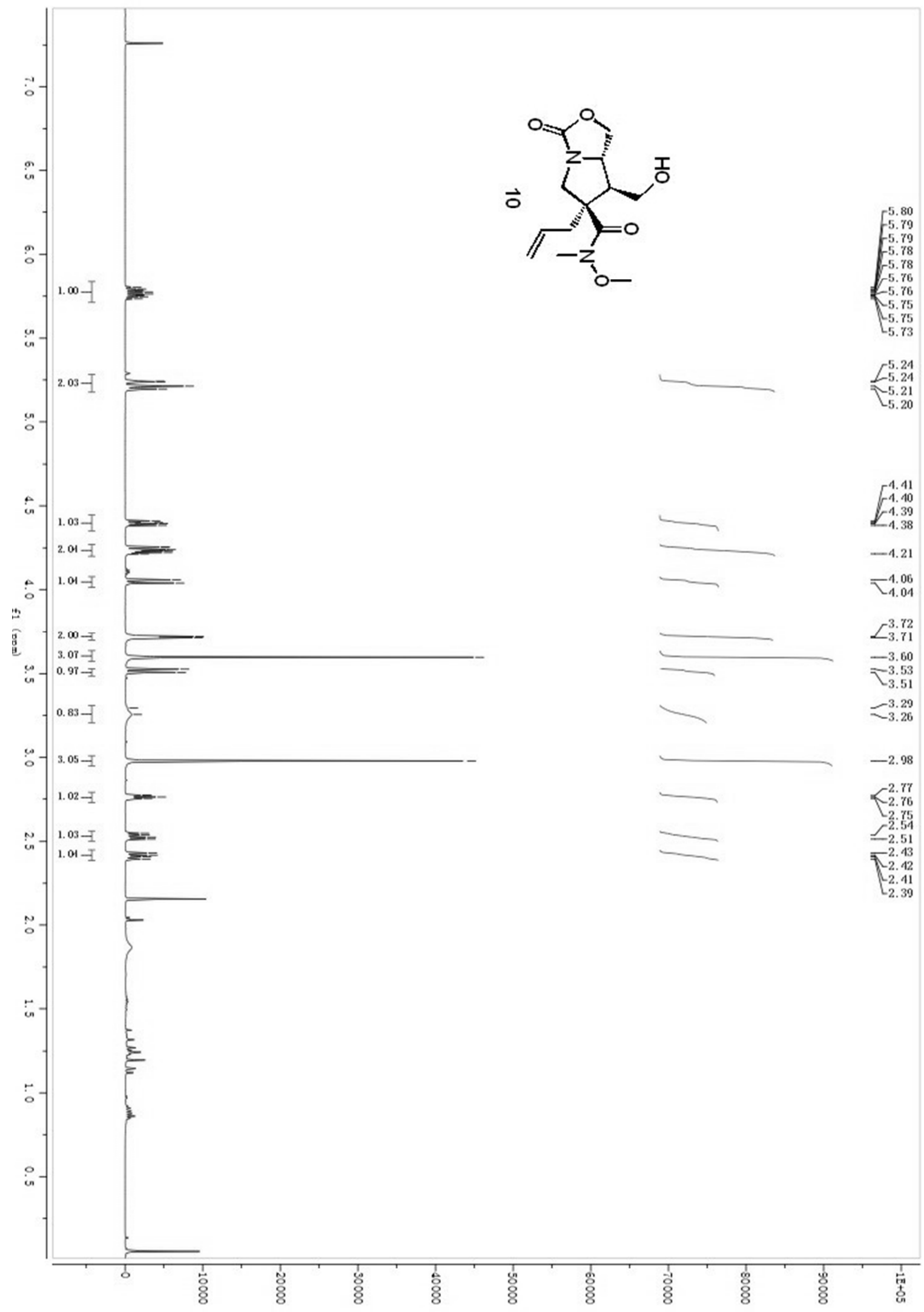




\section{Compound $10{ }^{13} \mathrm{C}$ NMR}

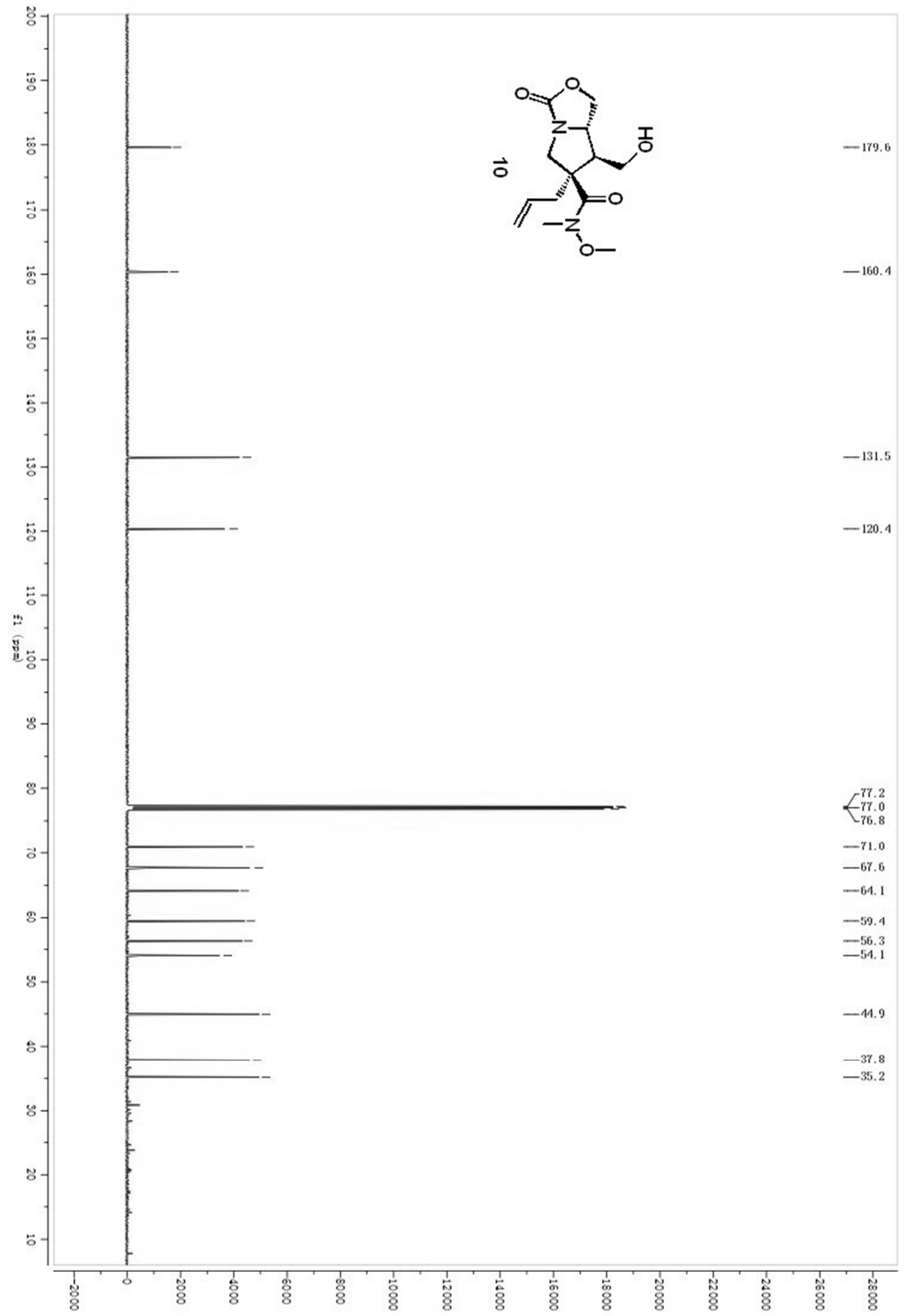




\section{Compound $11^{1} \mathrm{H}$ NMR}

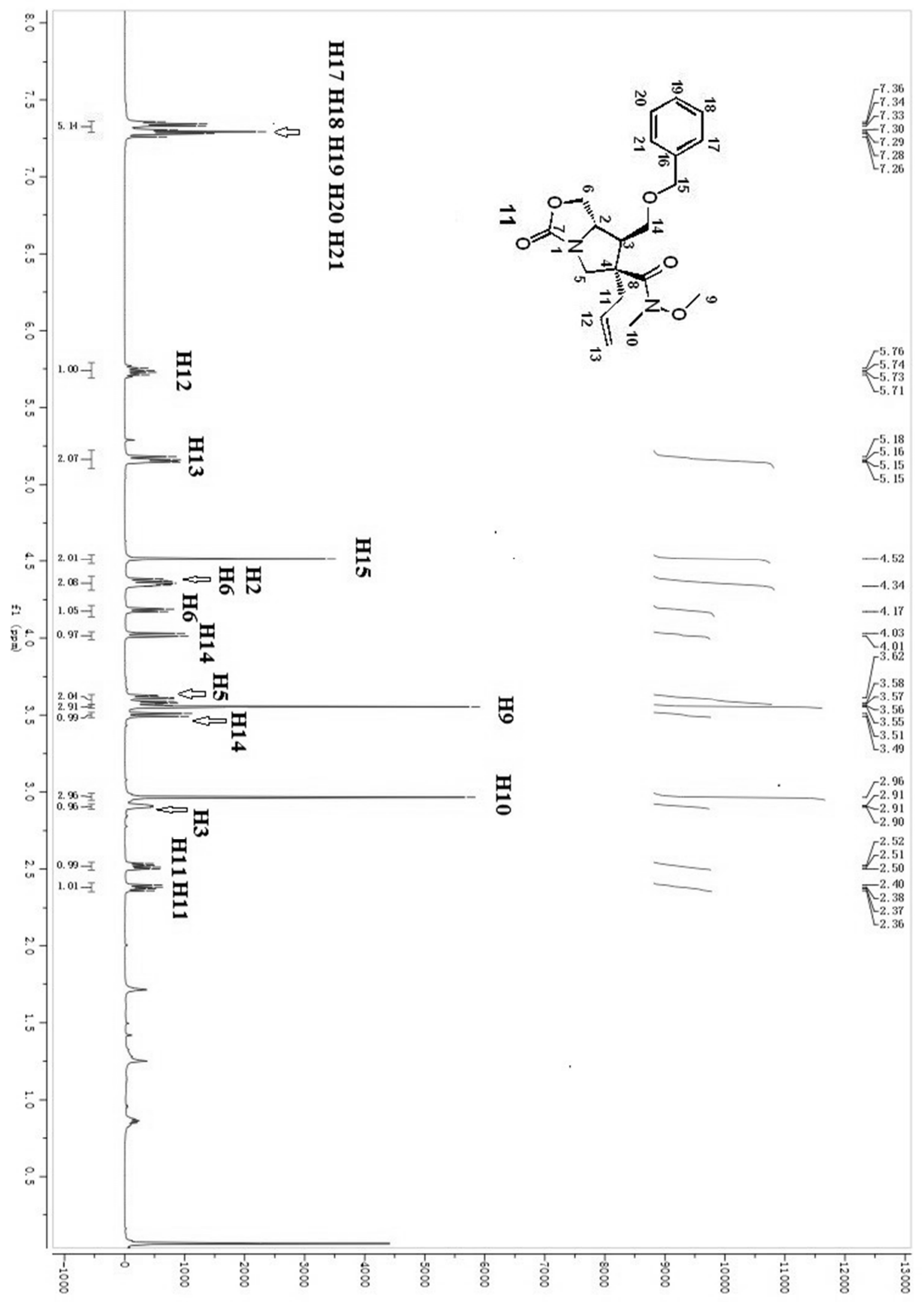




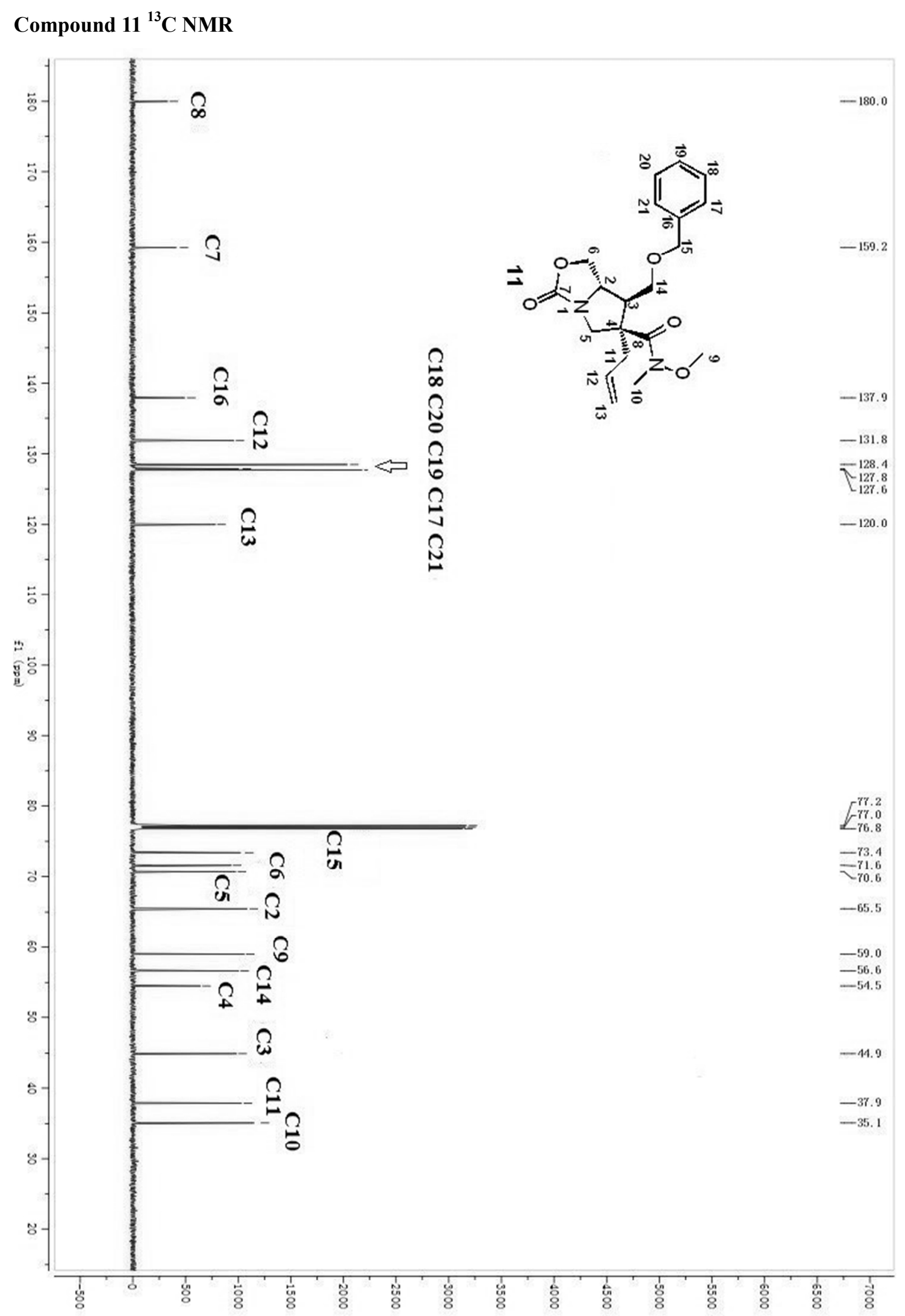




\section{Compound11 DEPT}

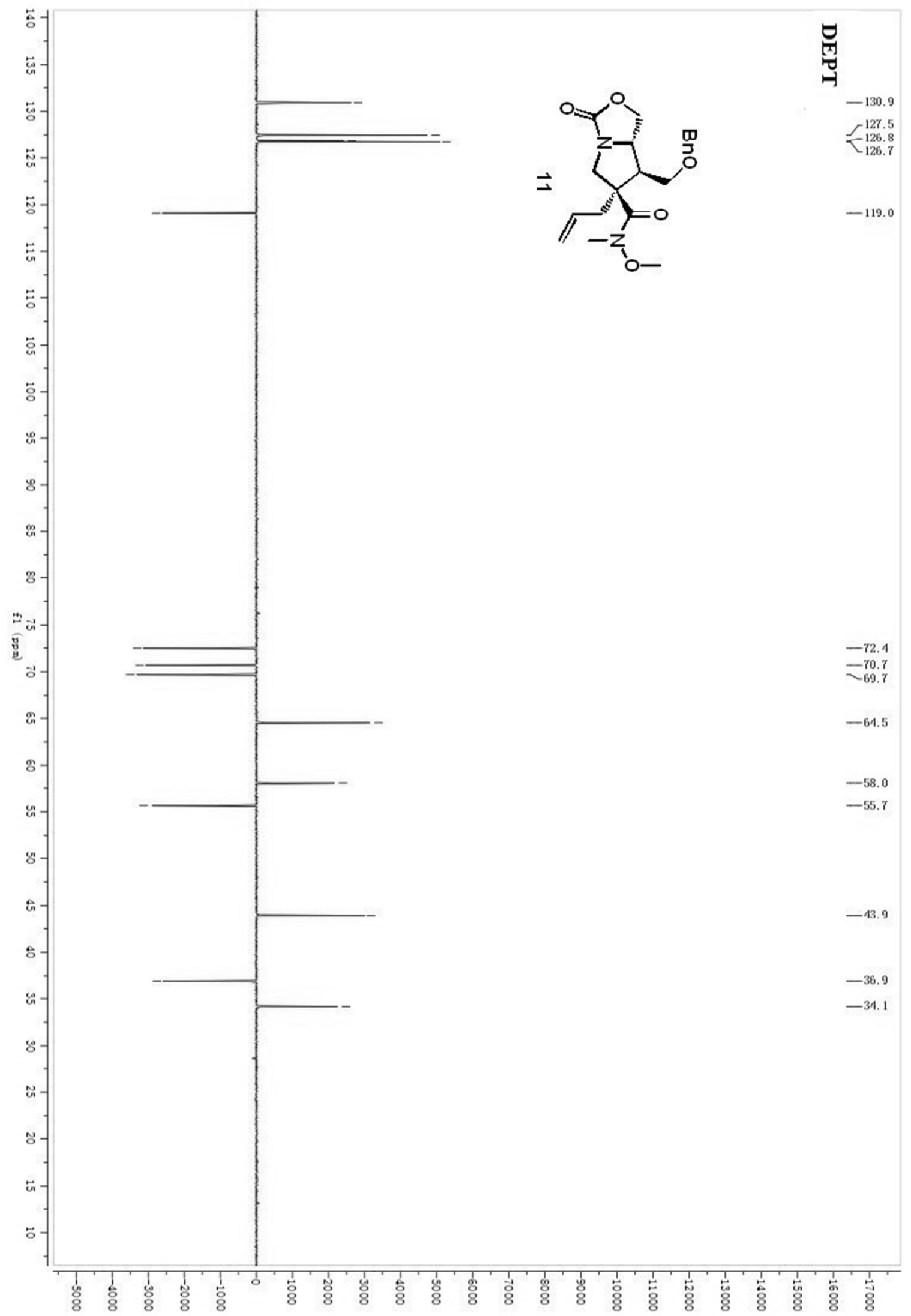




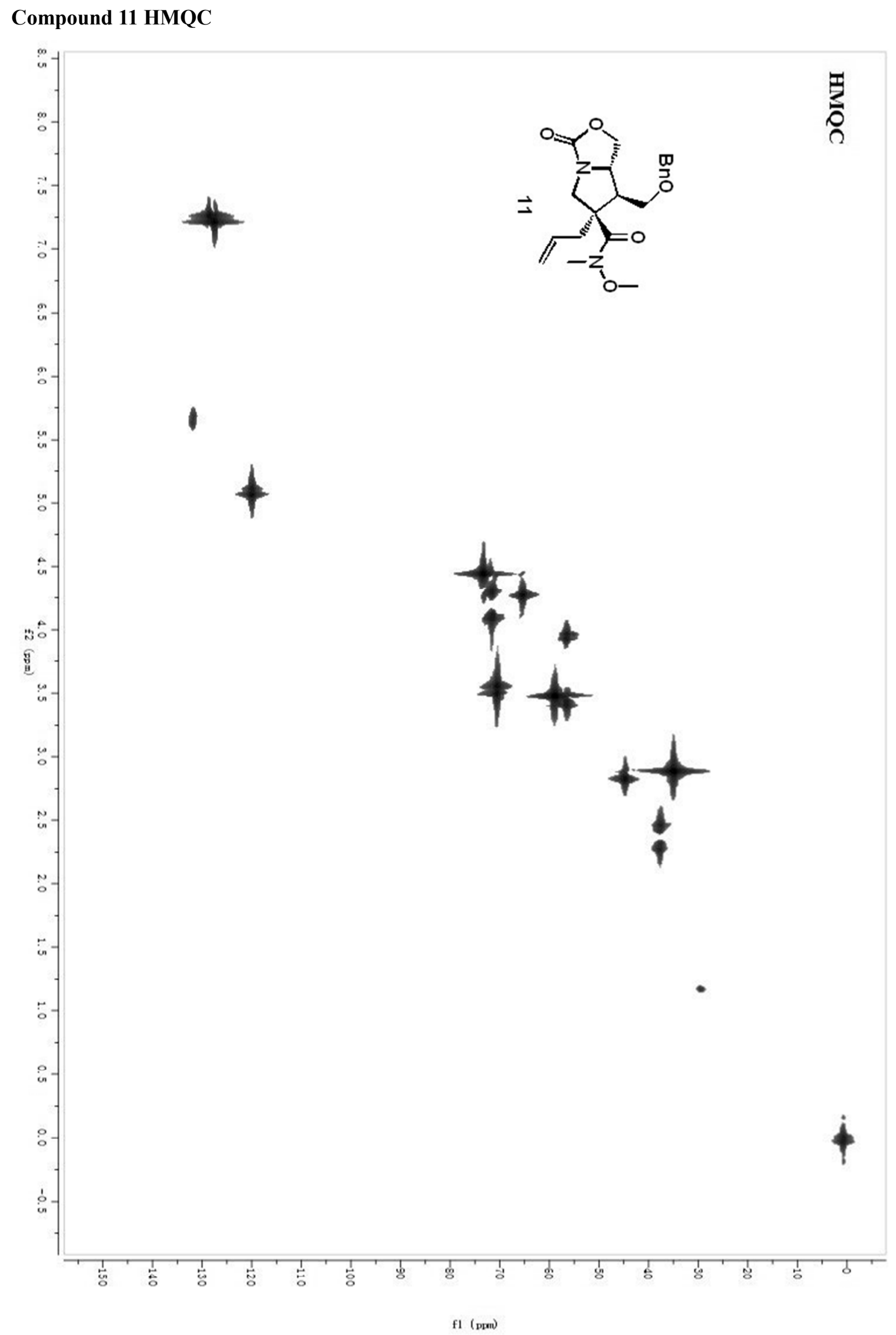

S-33 


\section{Compound 11 NOEDS}

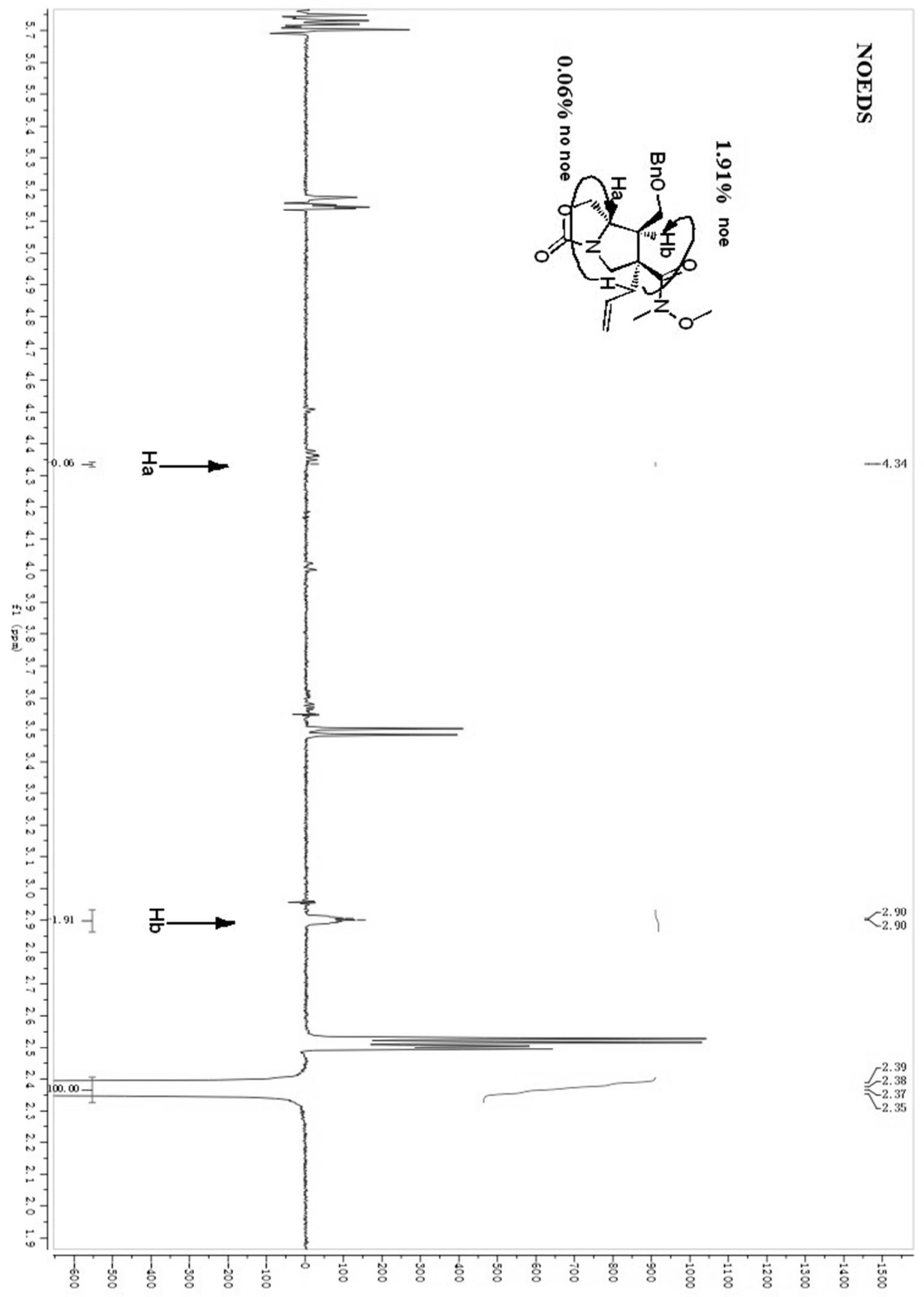




\section{Compound $13{ }^{1}$ H NMR}

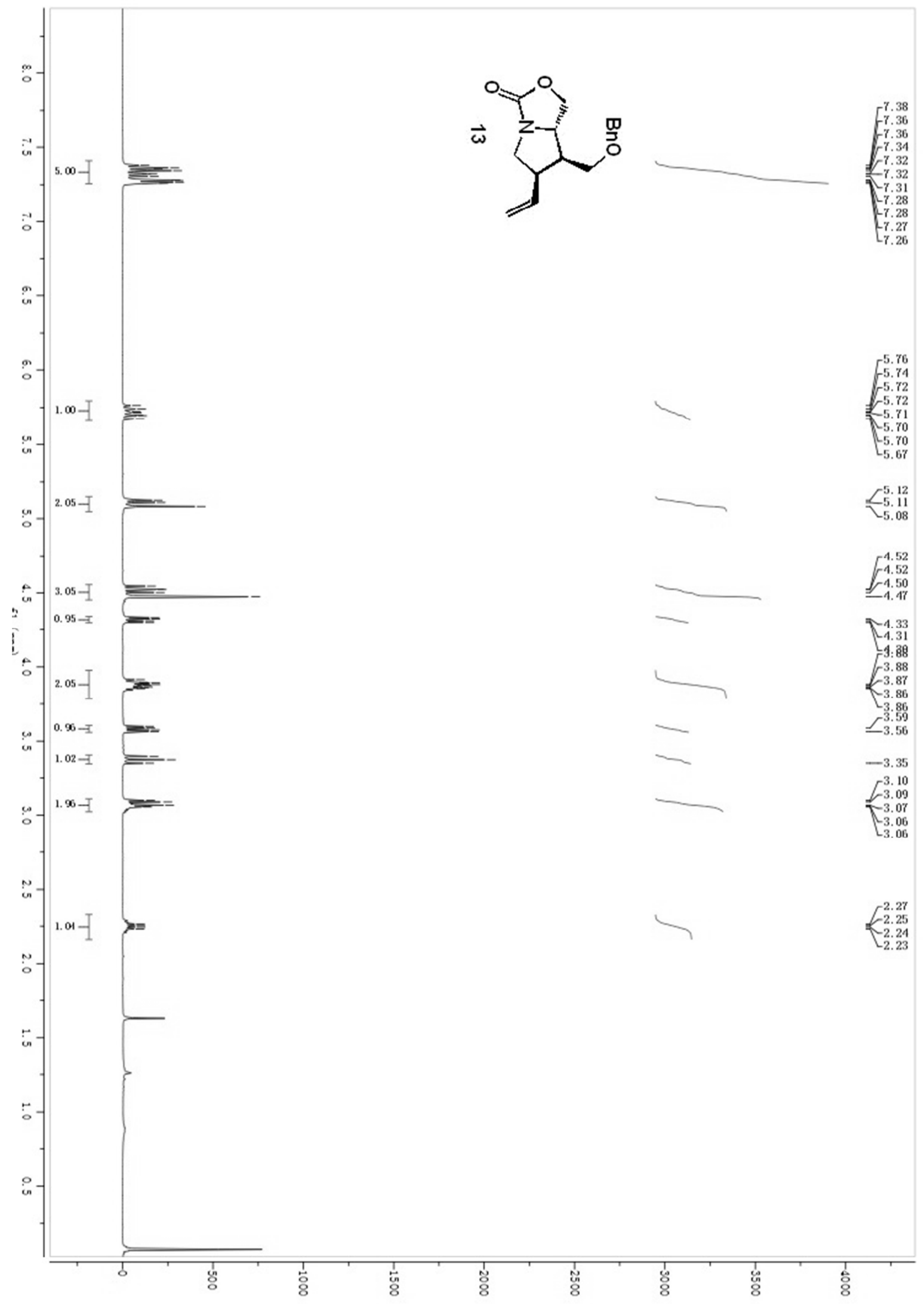




\section{Compound $13{ }^{13} \mathrm{C}$ NMR}

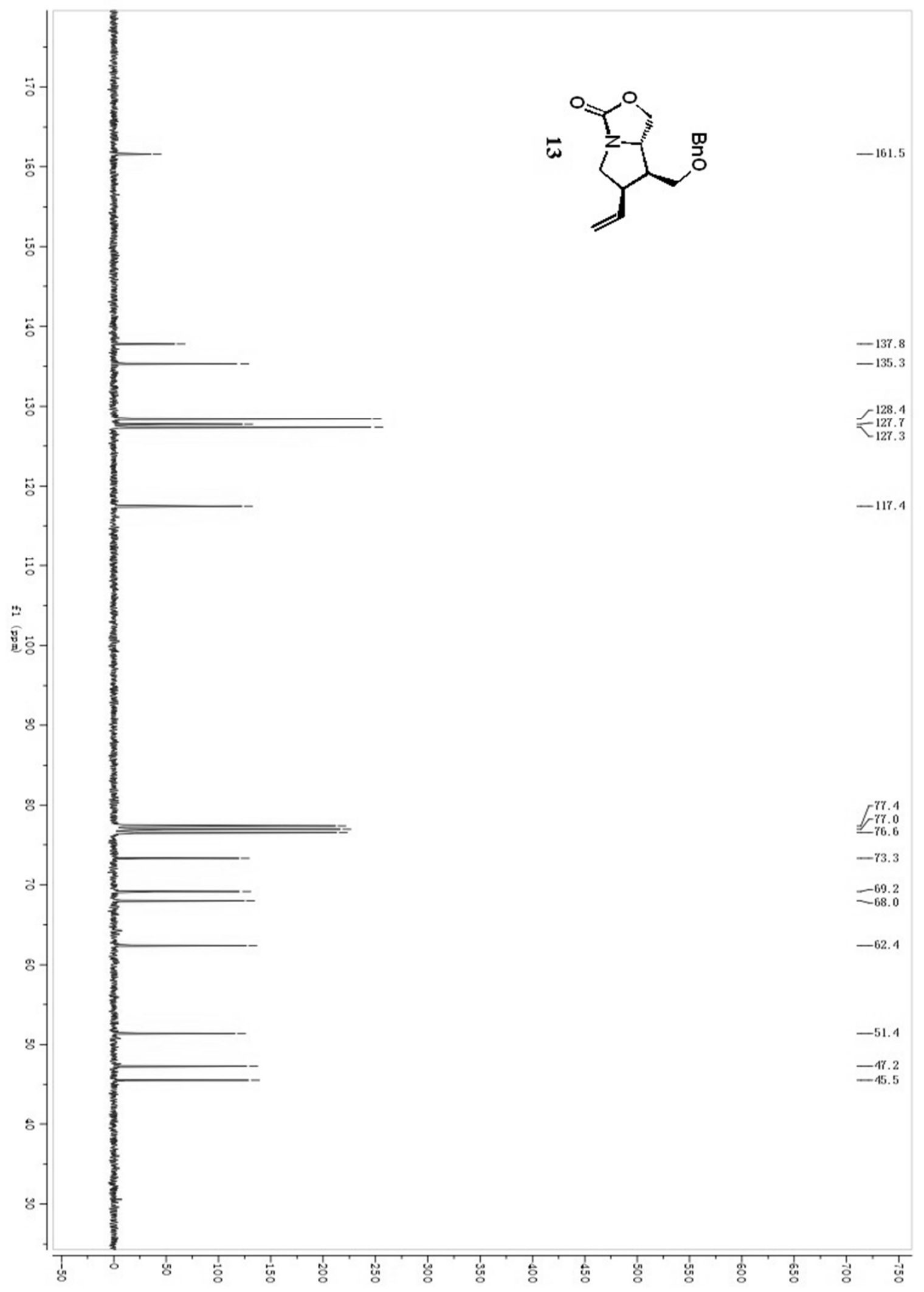




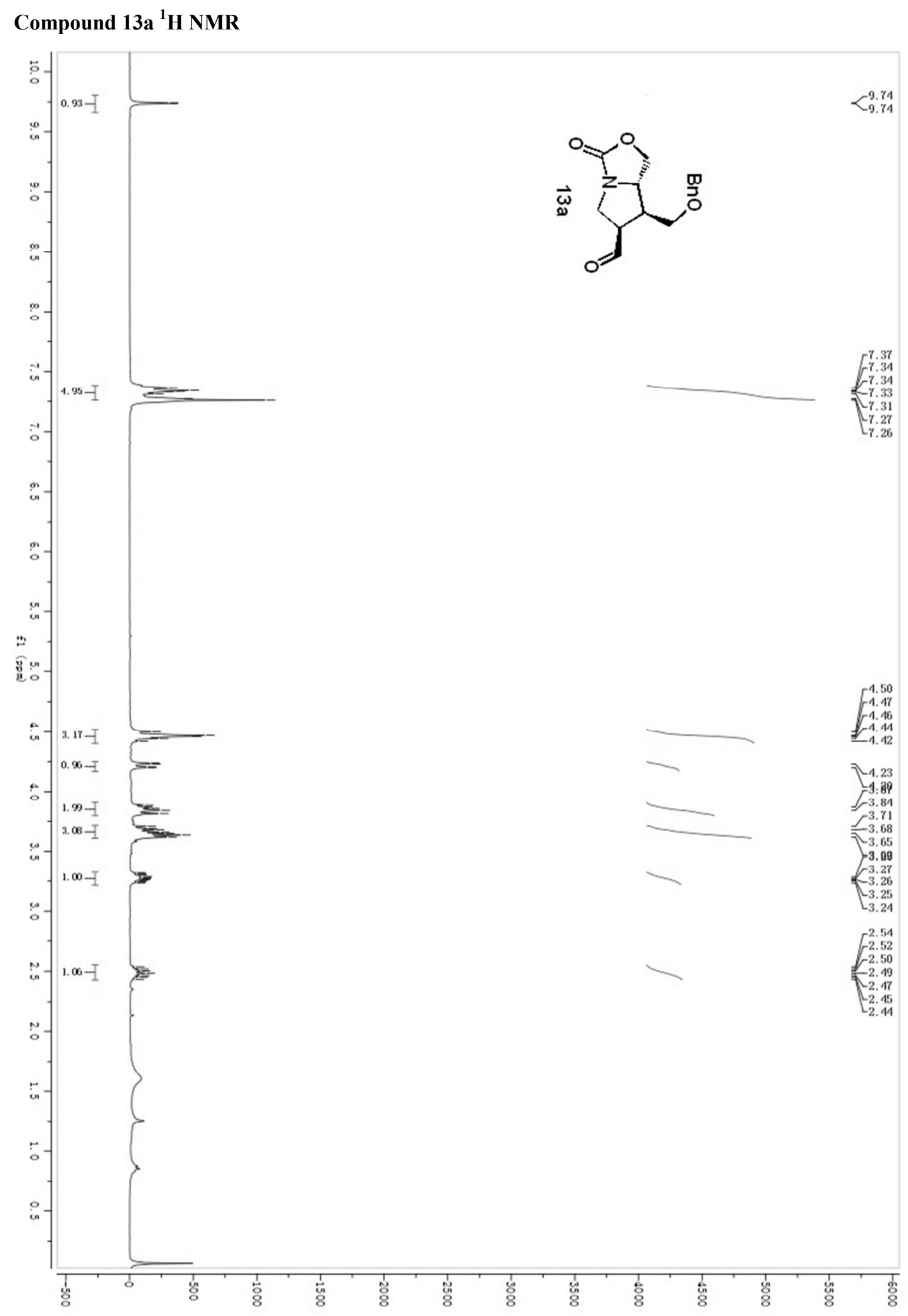




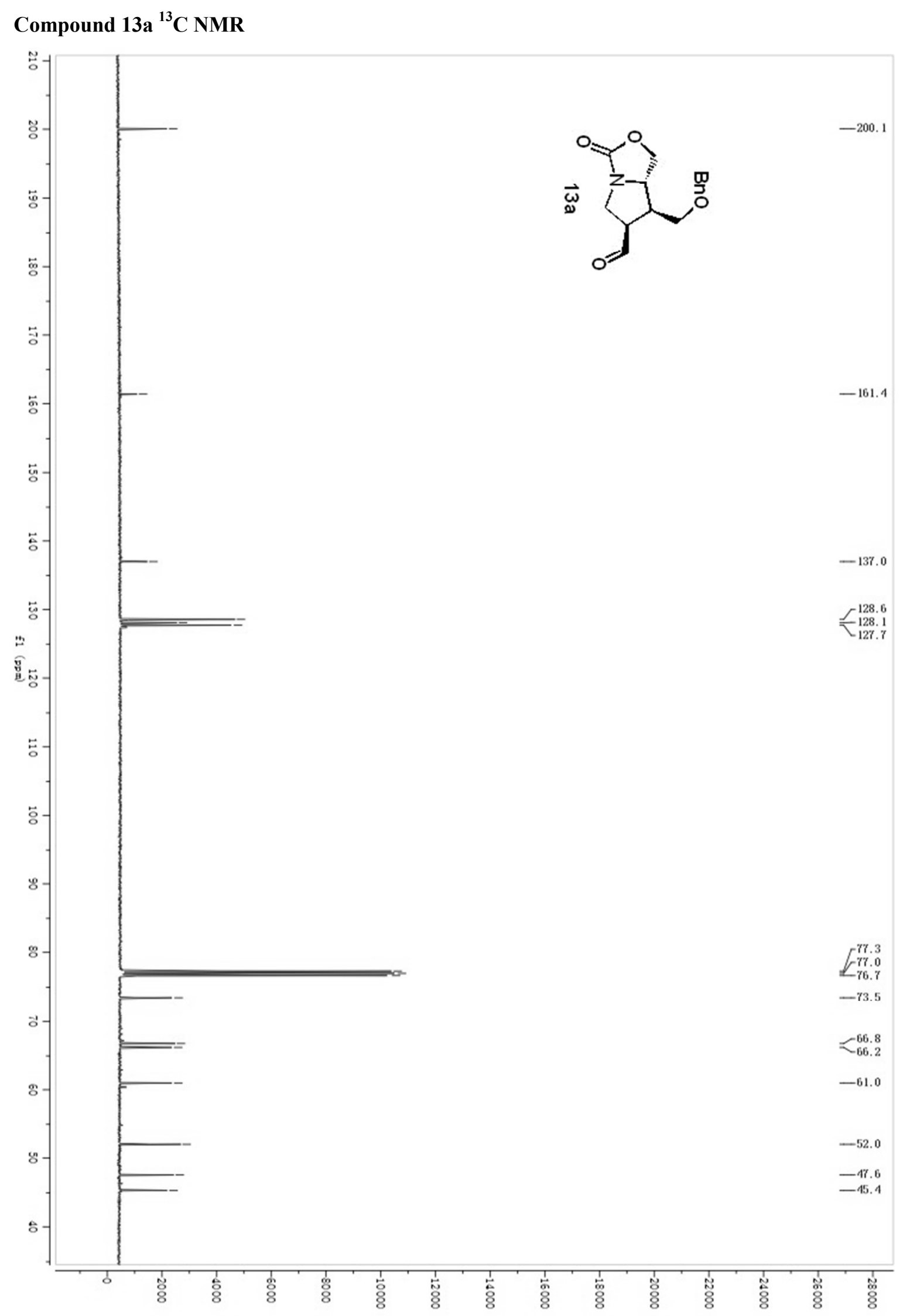




\section{Compound $14{ }^{1}$ H NMR}

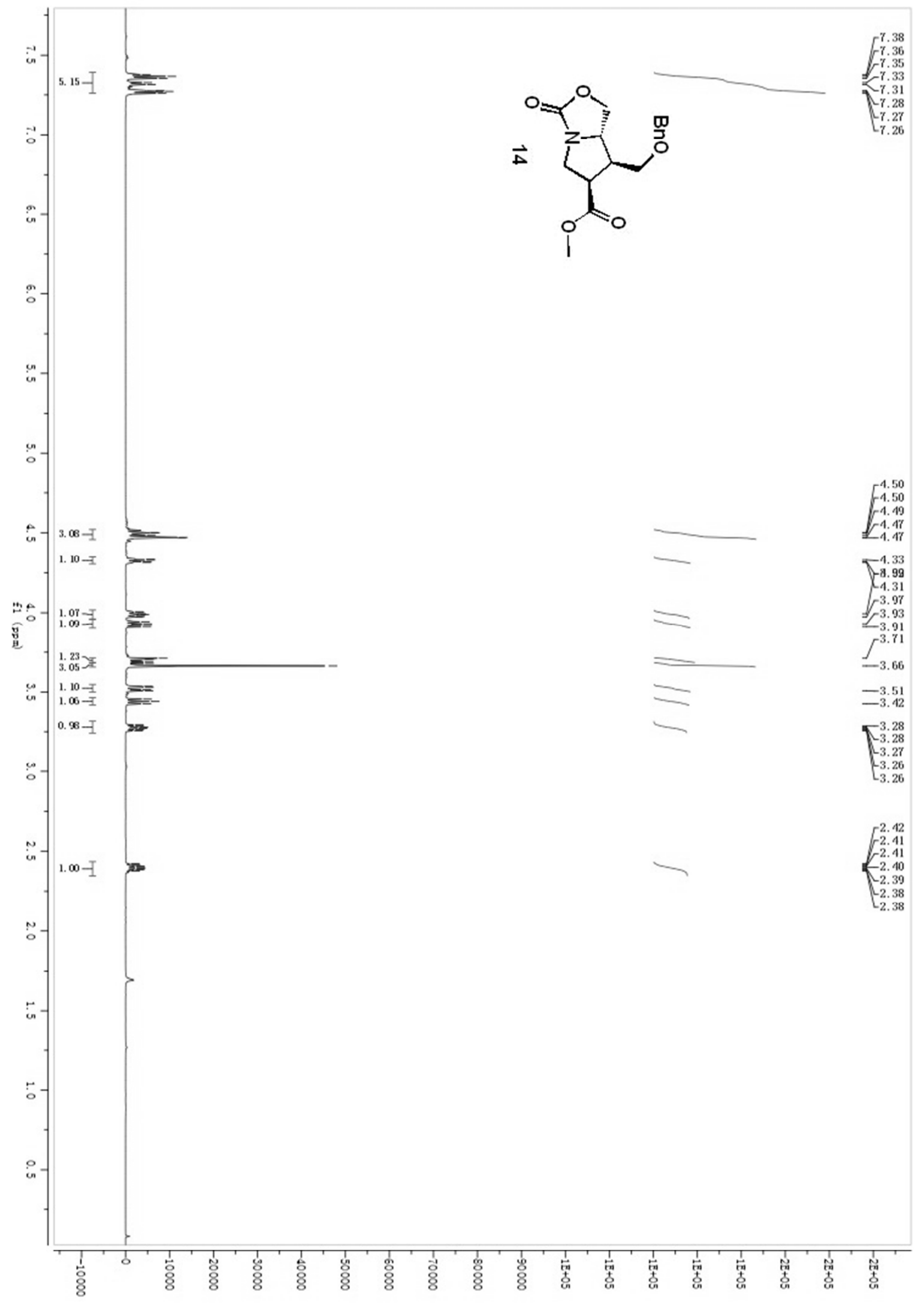




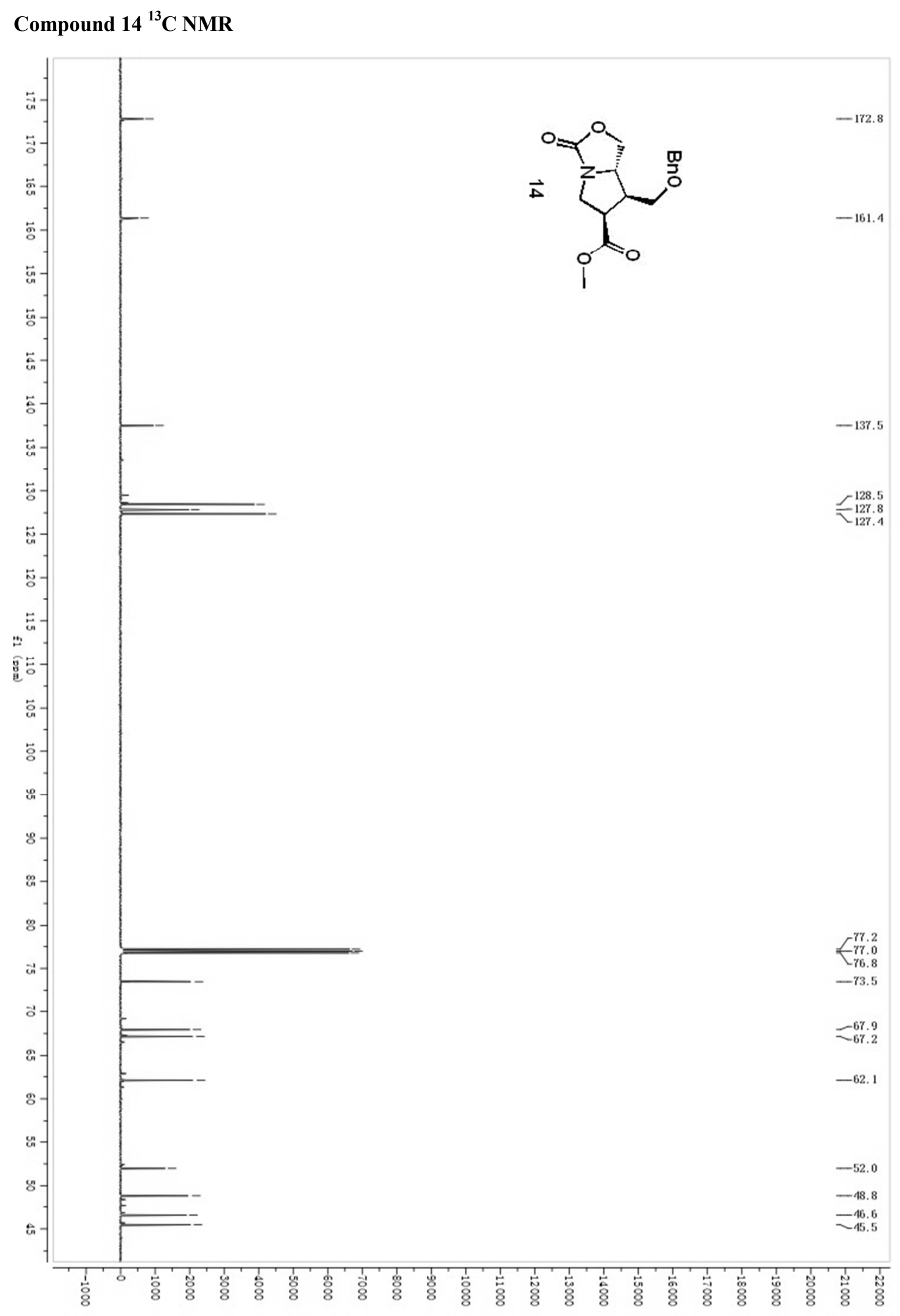




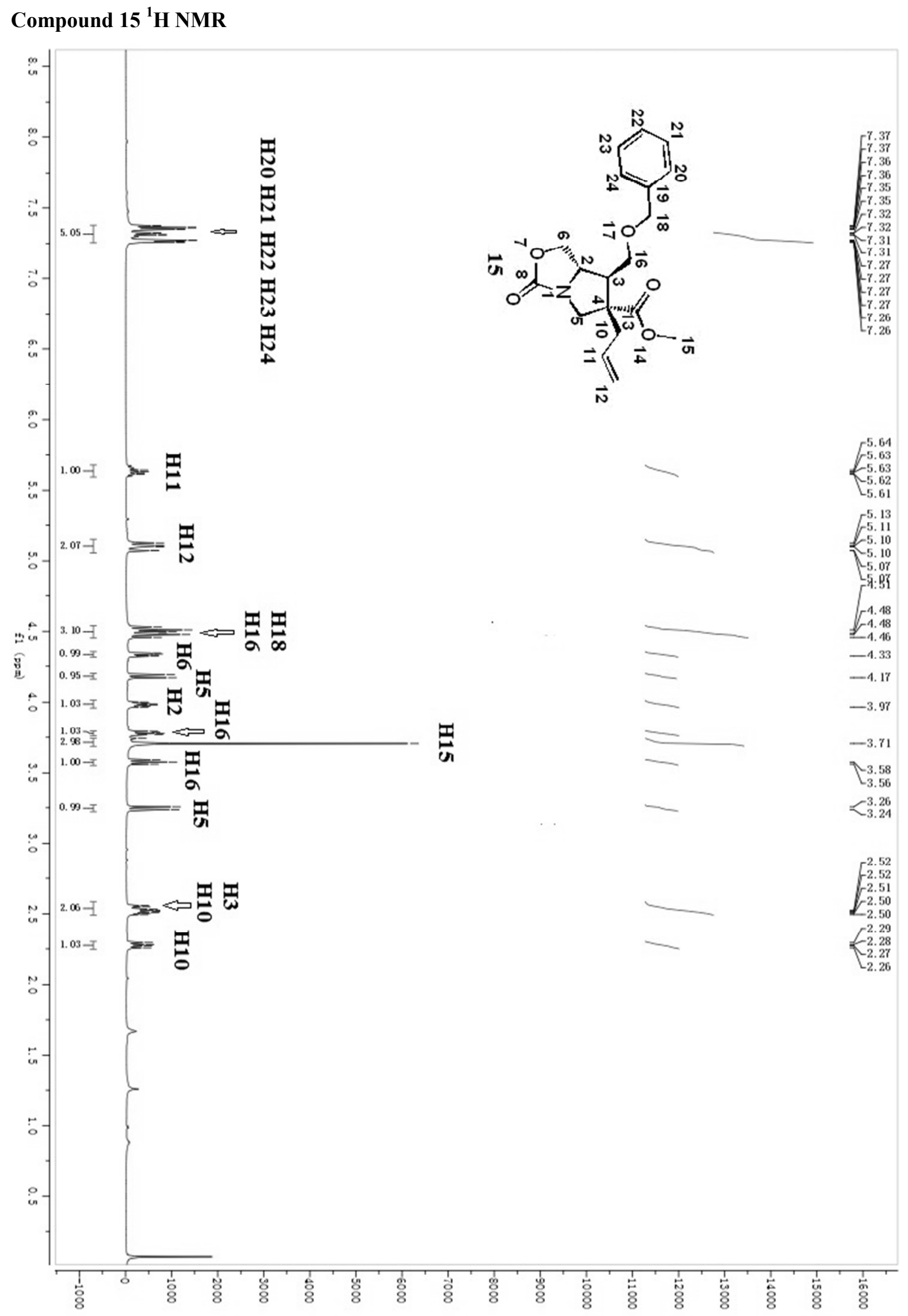


Compound $15{ }^{13} \mathrm{C}$ NMR

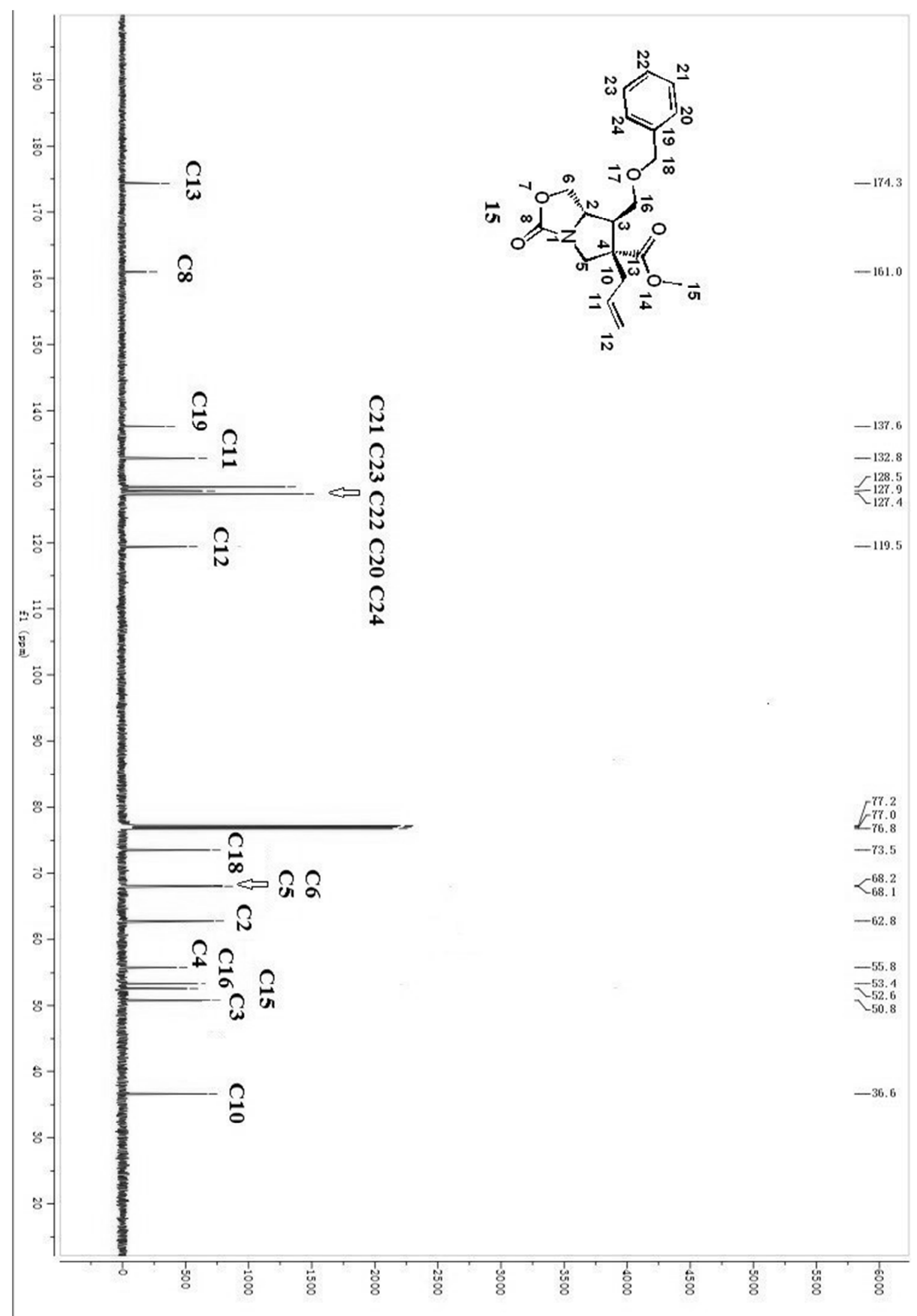




\section{Compound 15 DEPT}

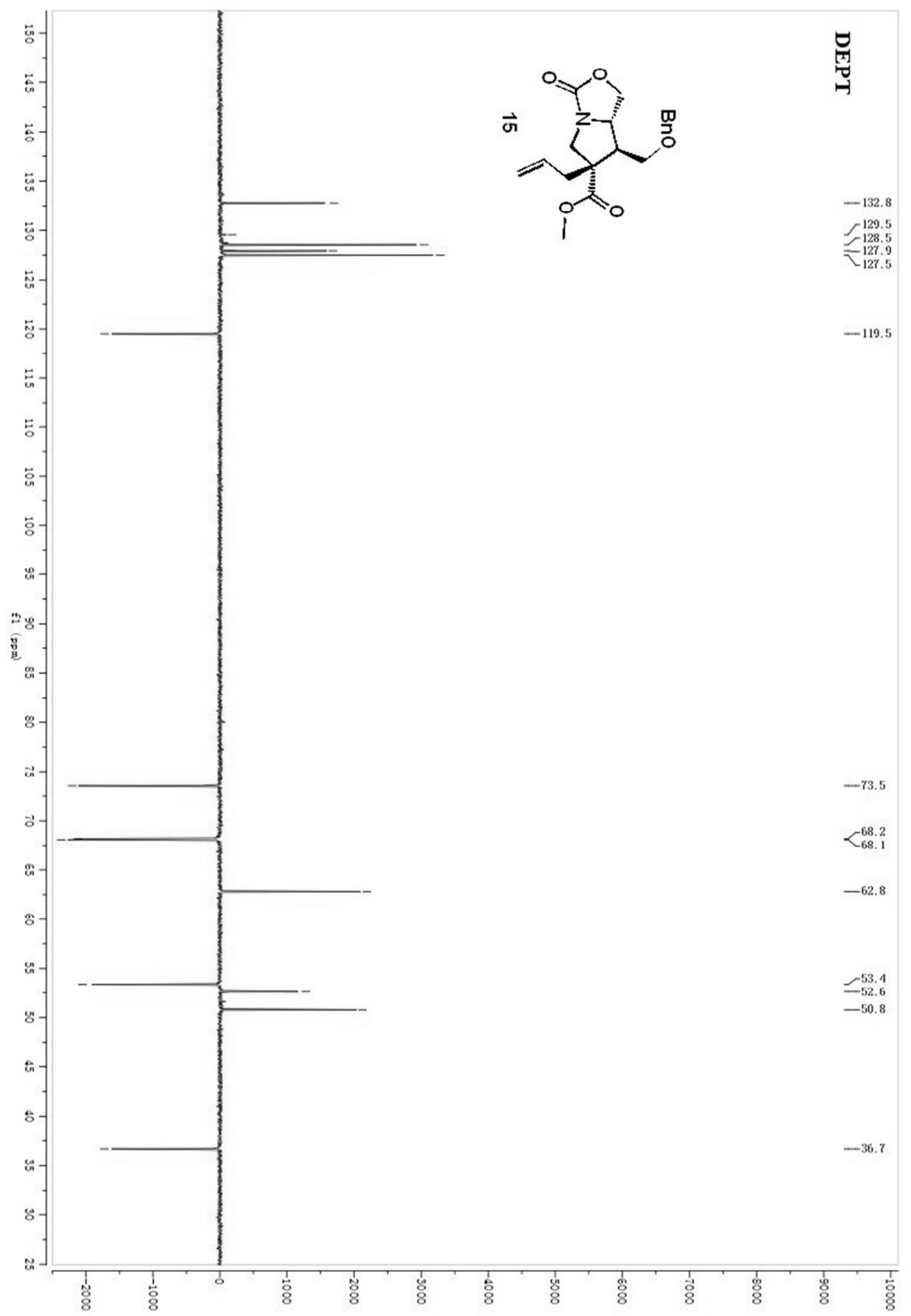




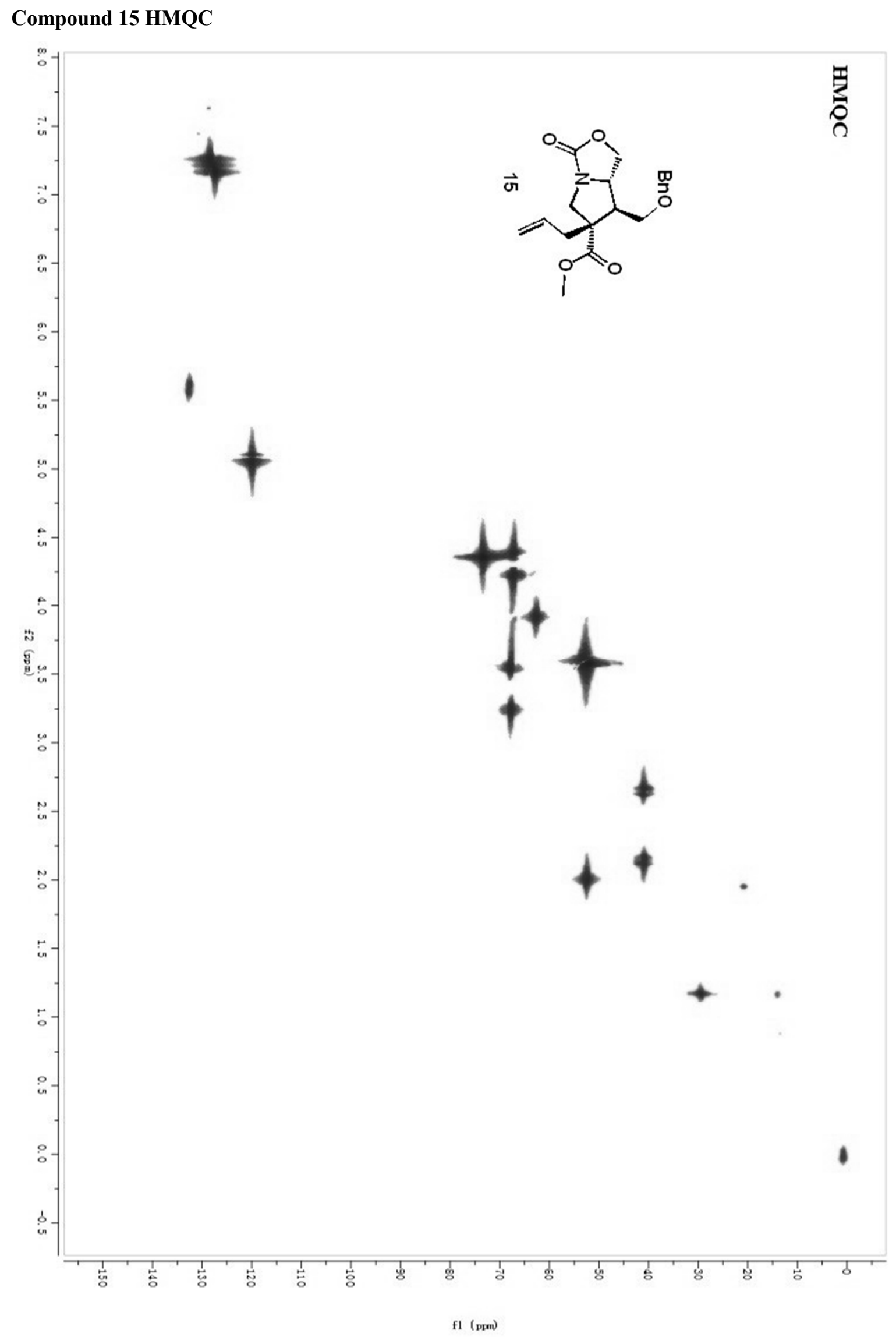




\section{Compound 15 NOEDS}

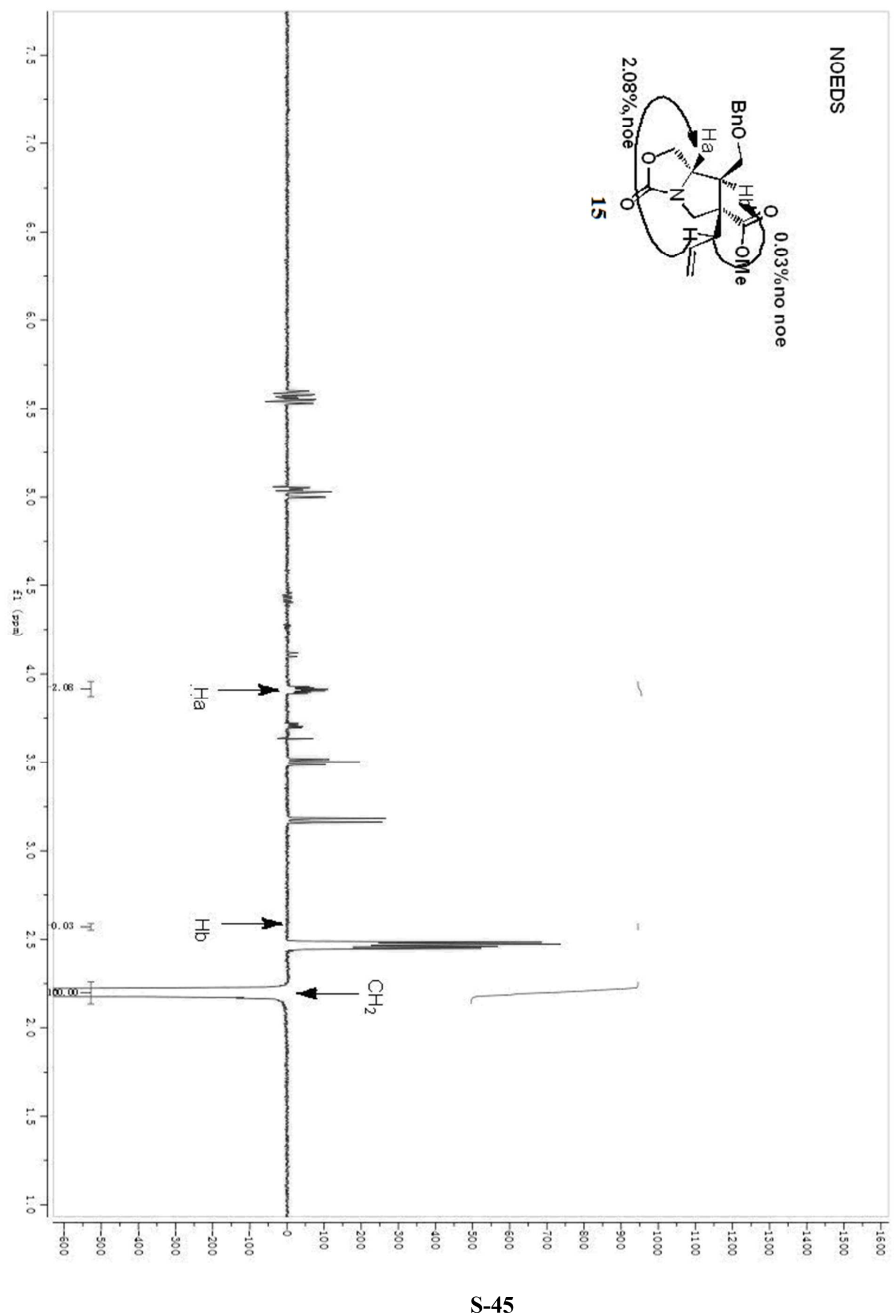




\section{Compound $16{ }^{1}$ H NMR}

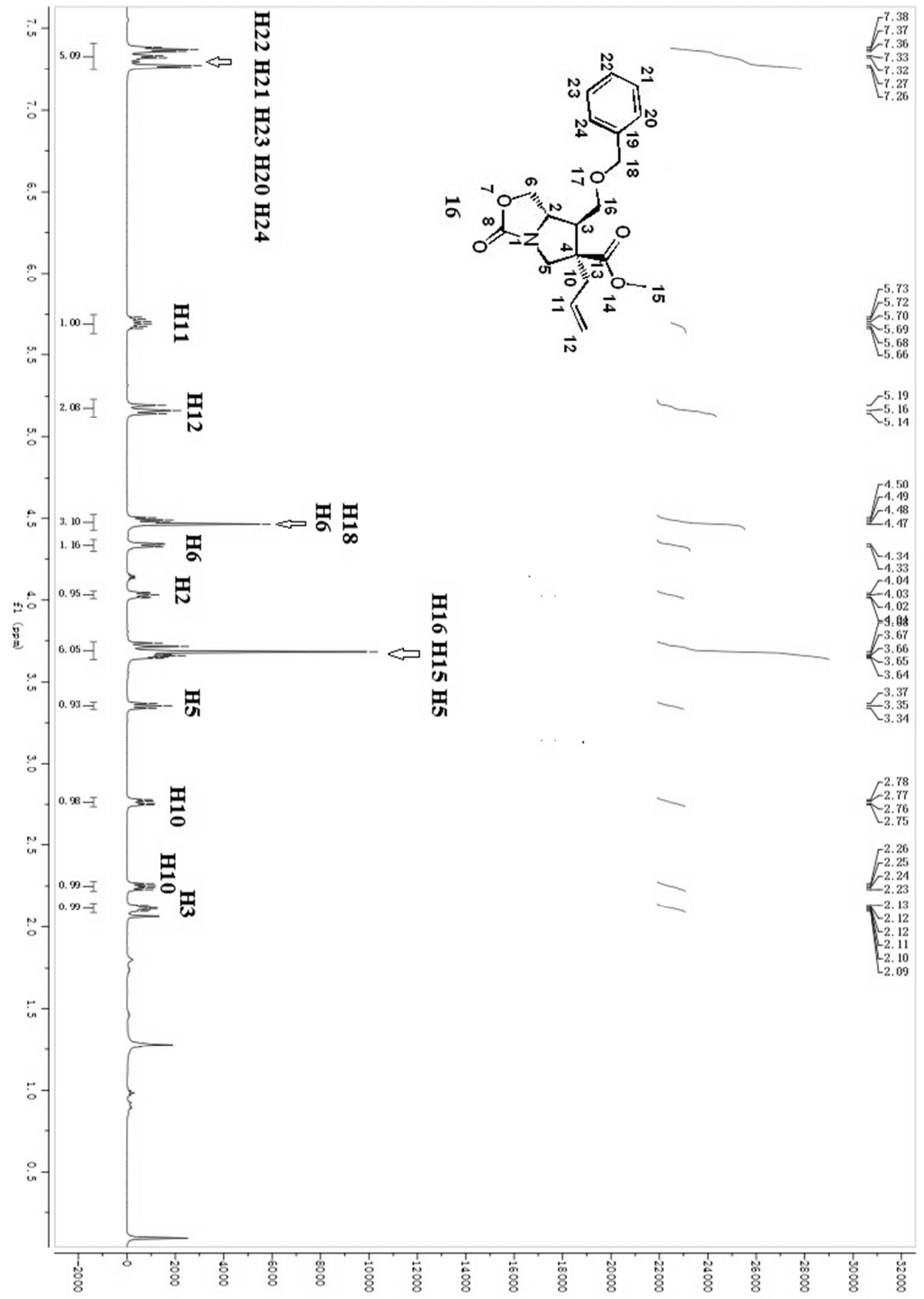




\section{Compound $16{ }^{13} \mathrm{C}$ NMR}

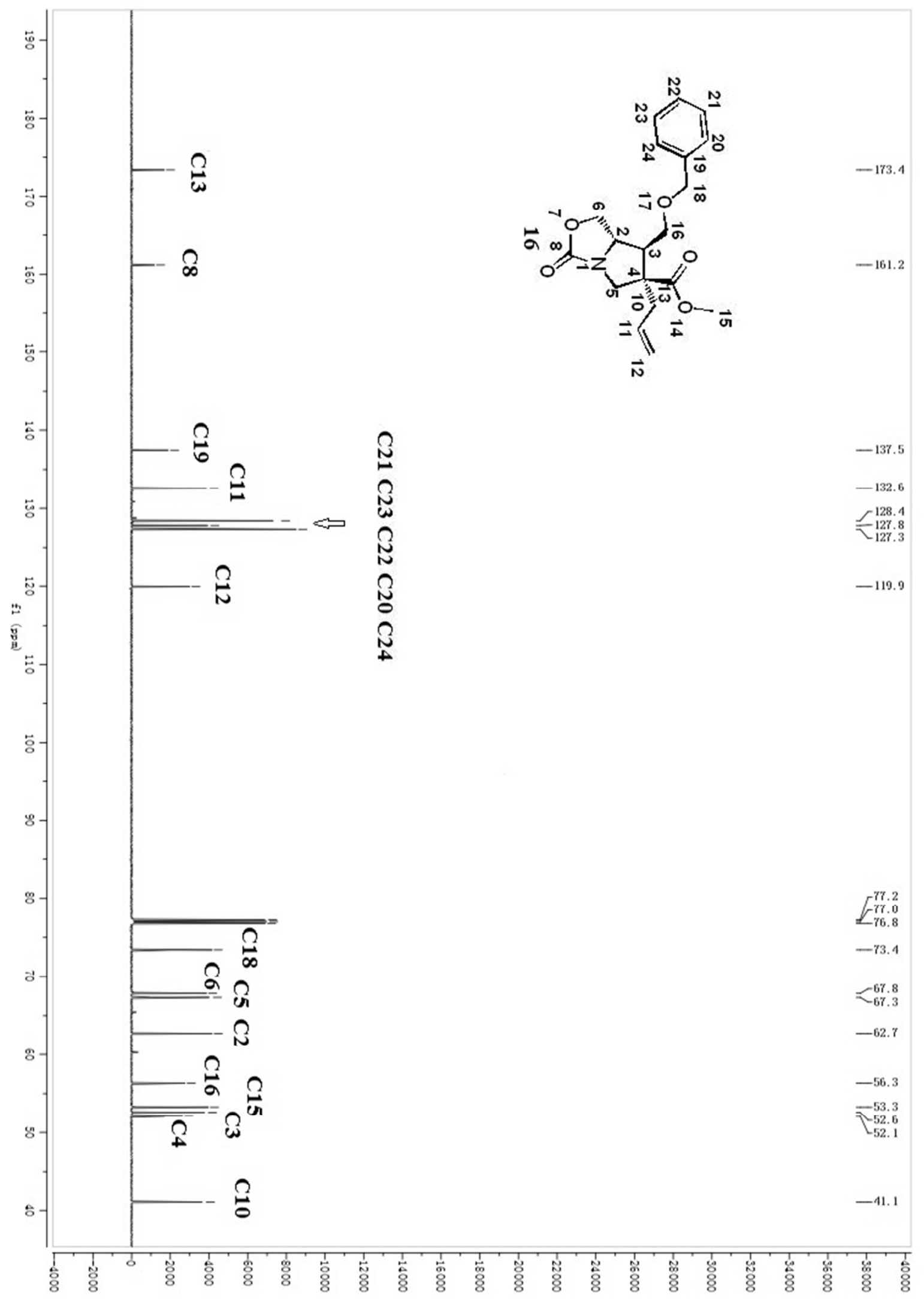




\section{Compound 16 DEPT}

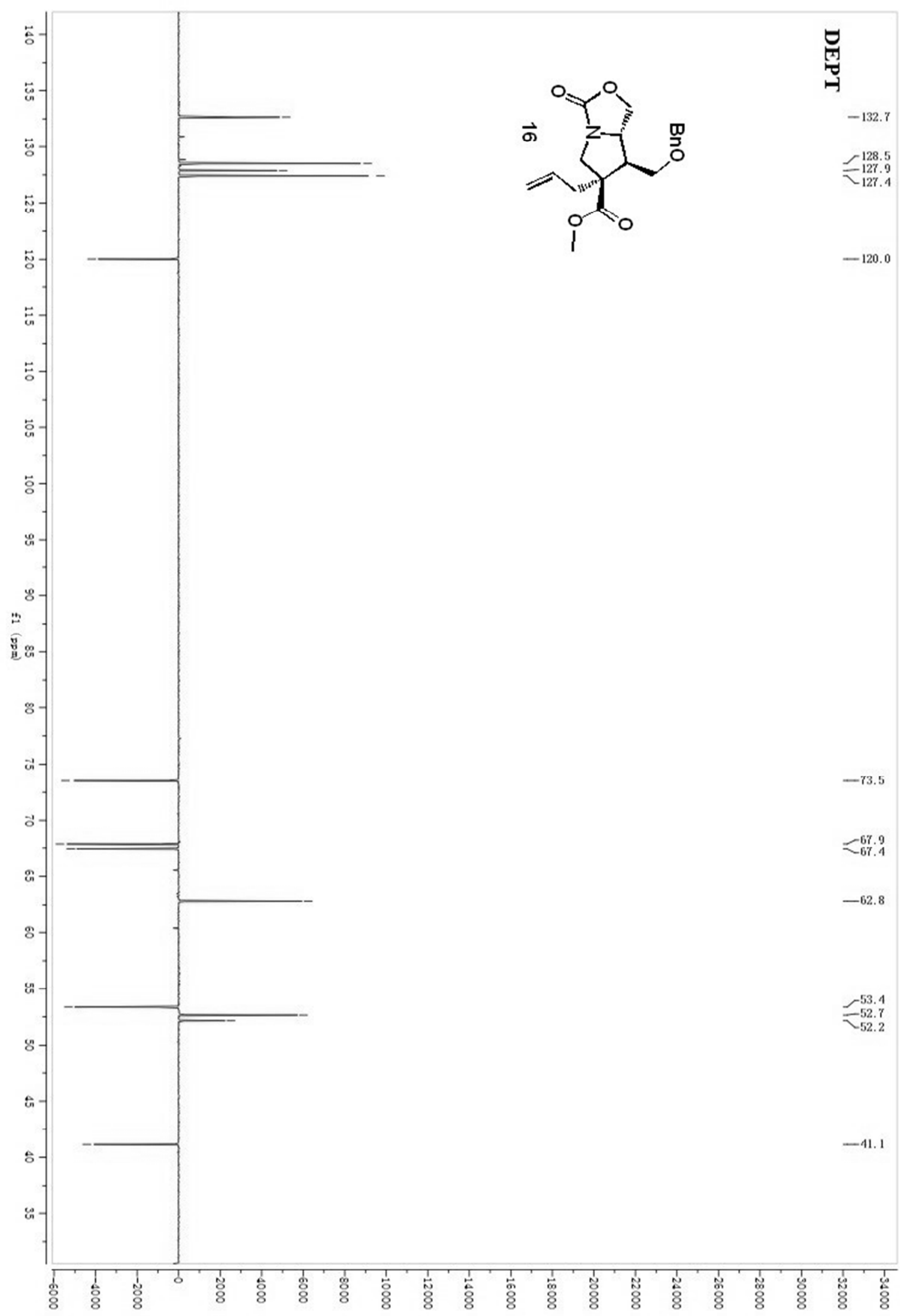




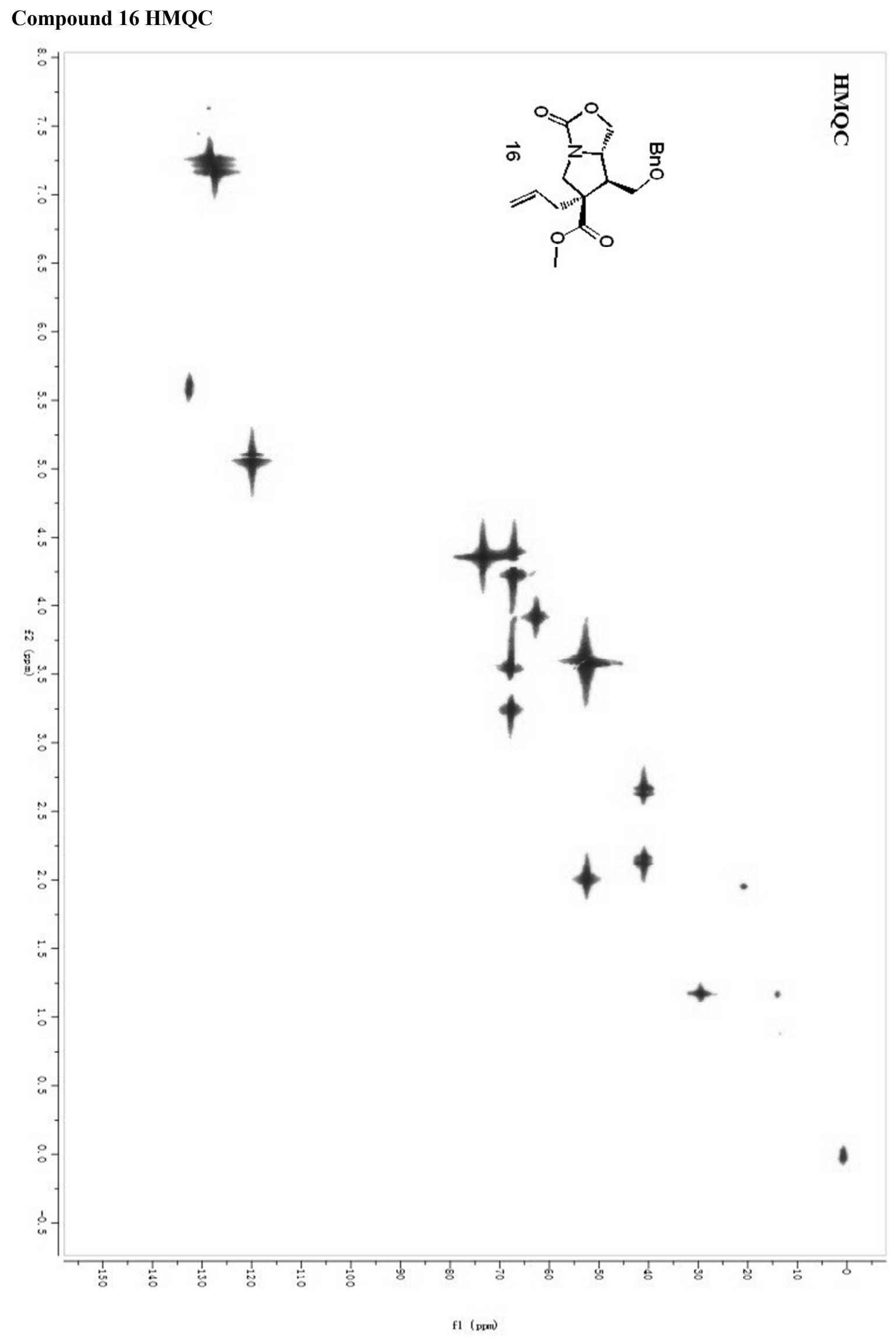




\section{Compound 16 NOEDS}

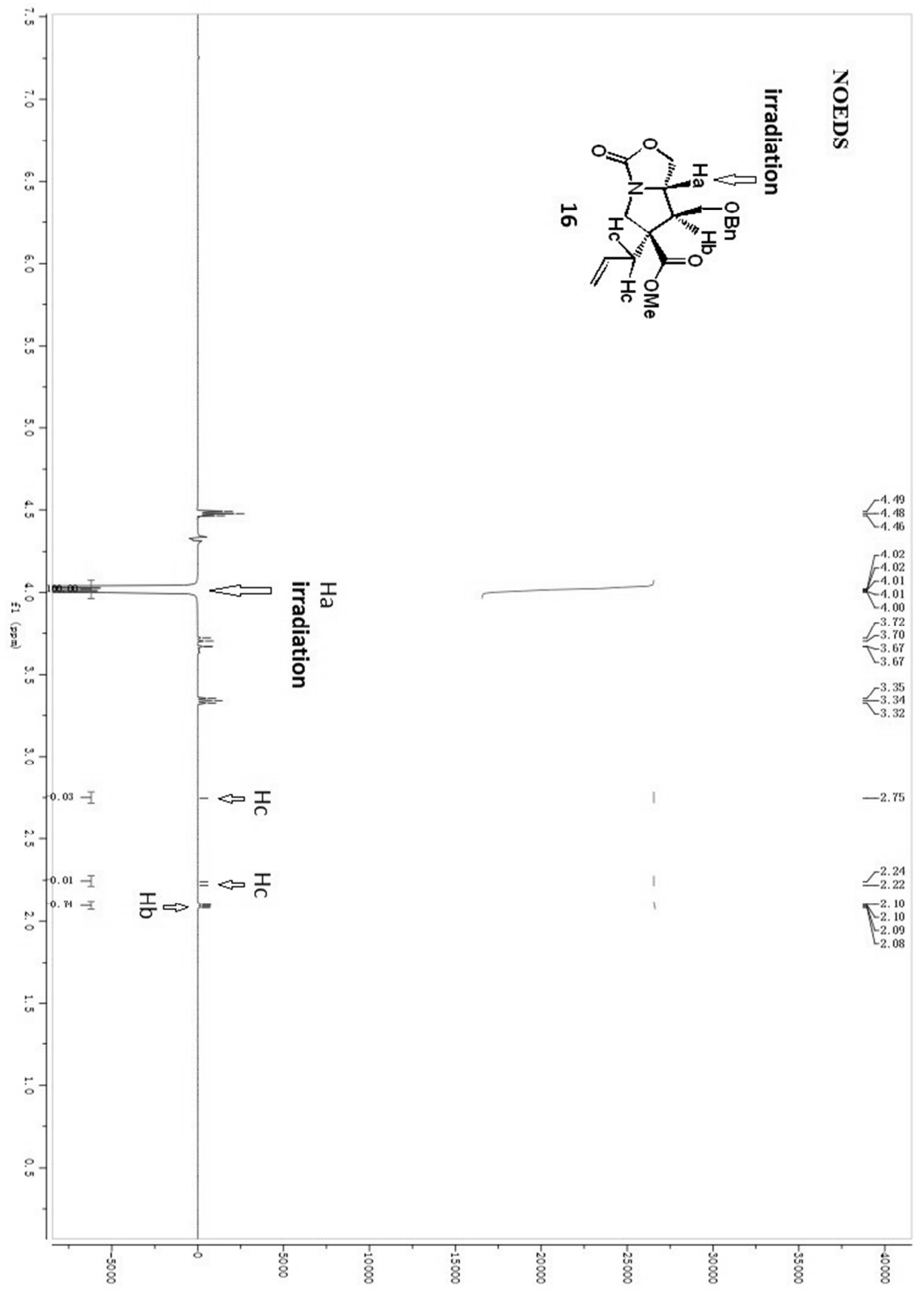




\section{Compound $12{ }^{1}$ H NMR}

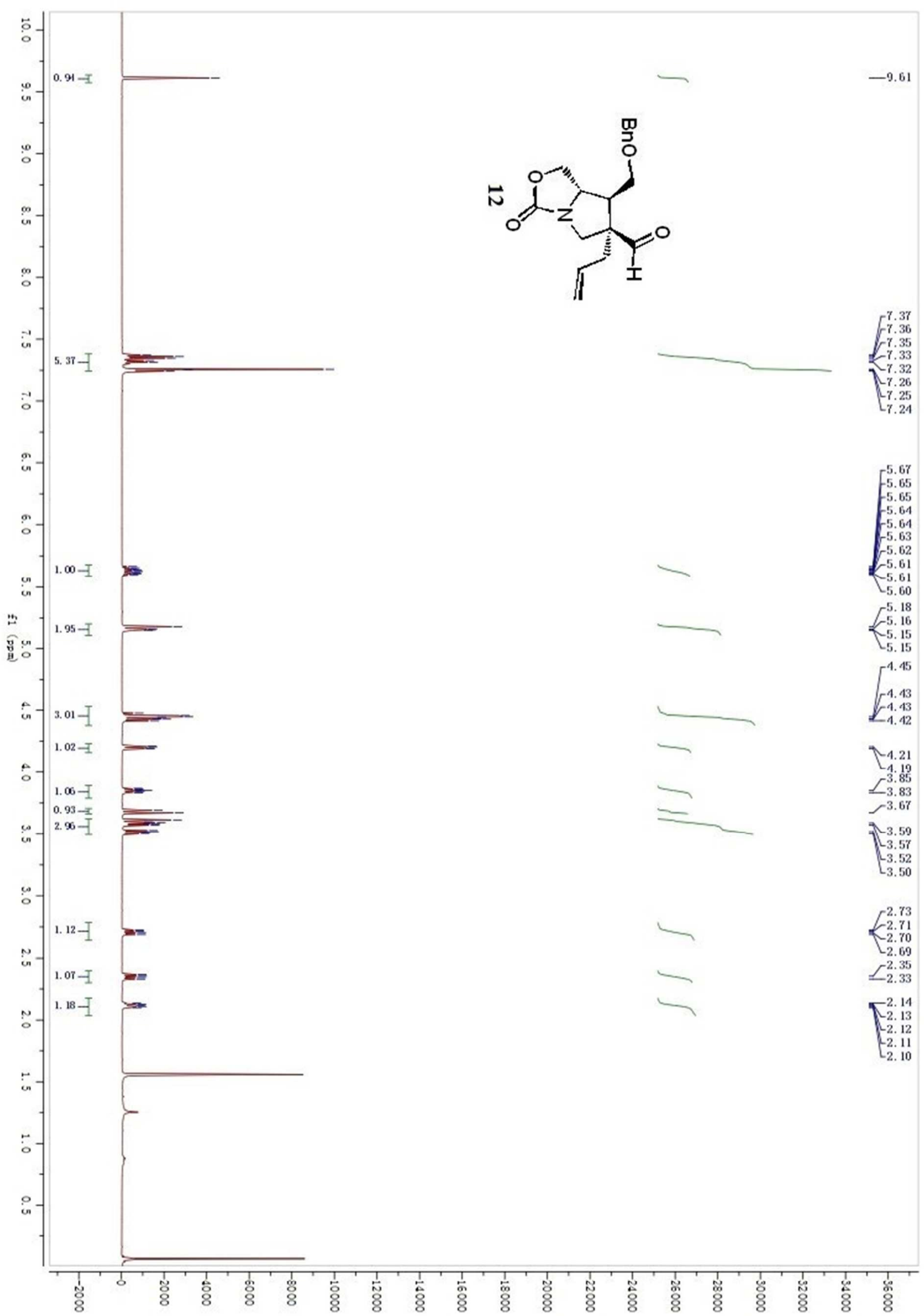


Compound $12{ }^{13} \mathrm{C}$ NMR

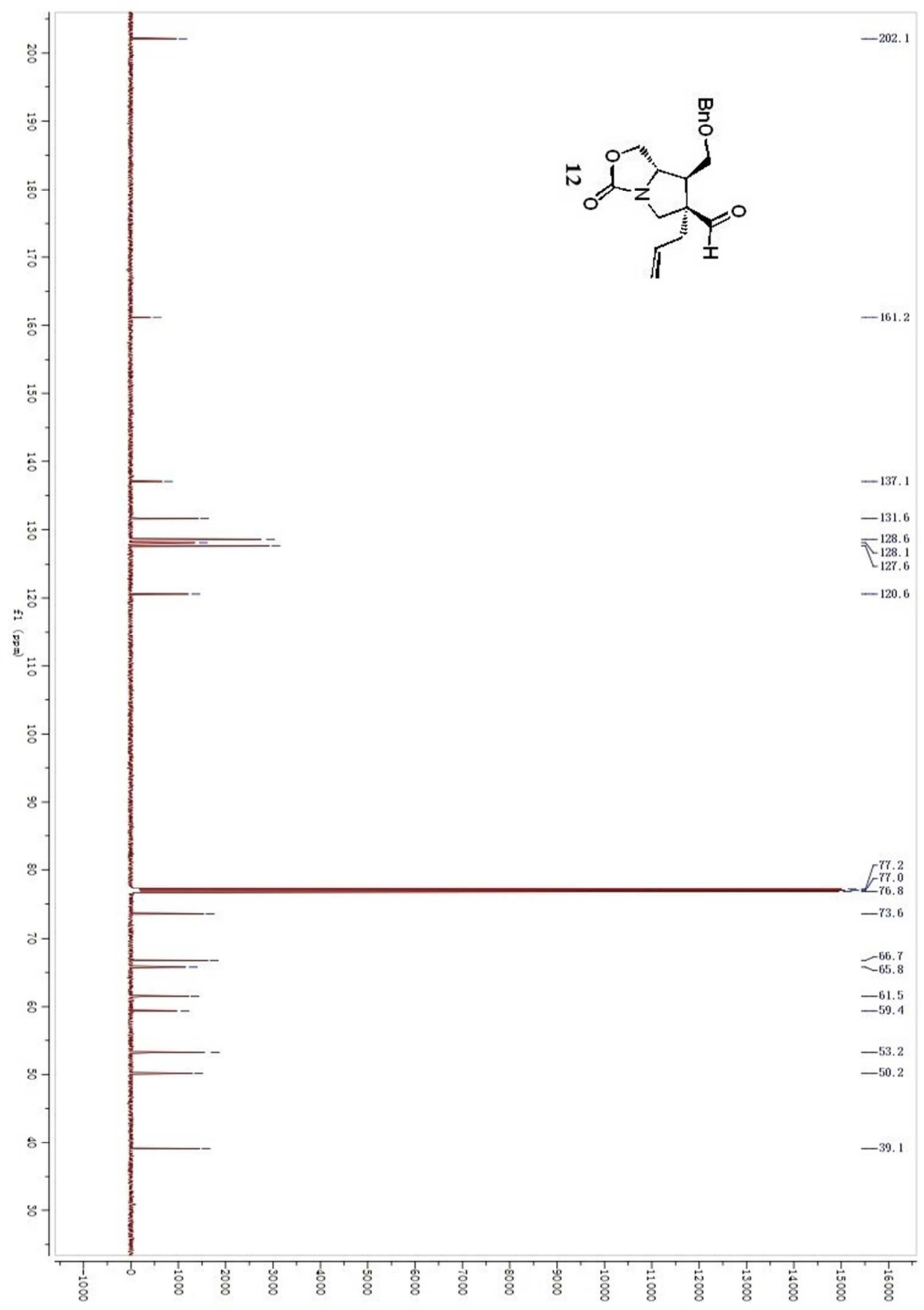




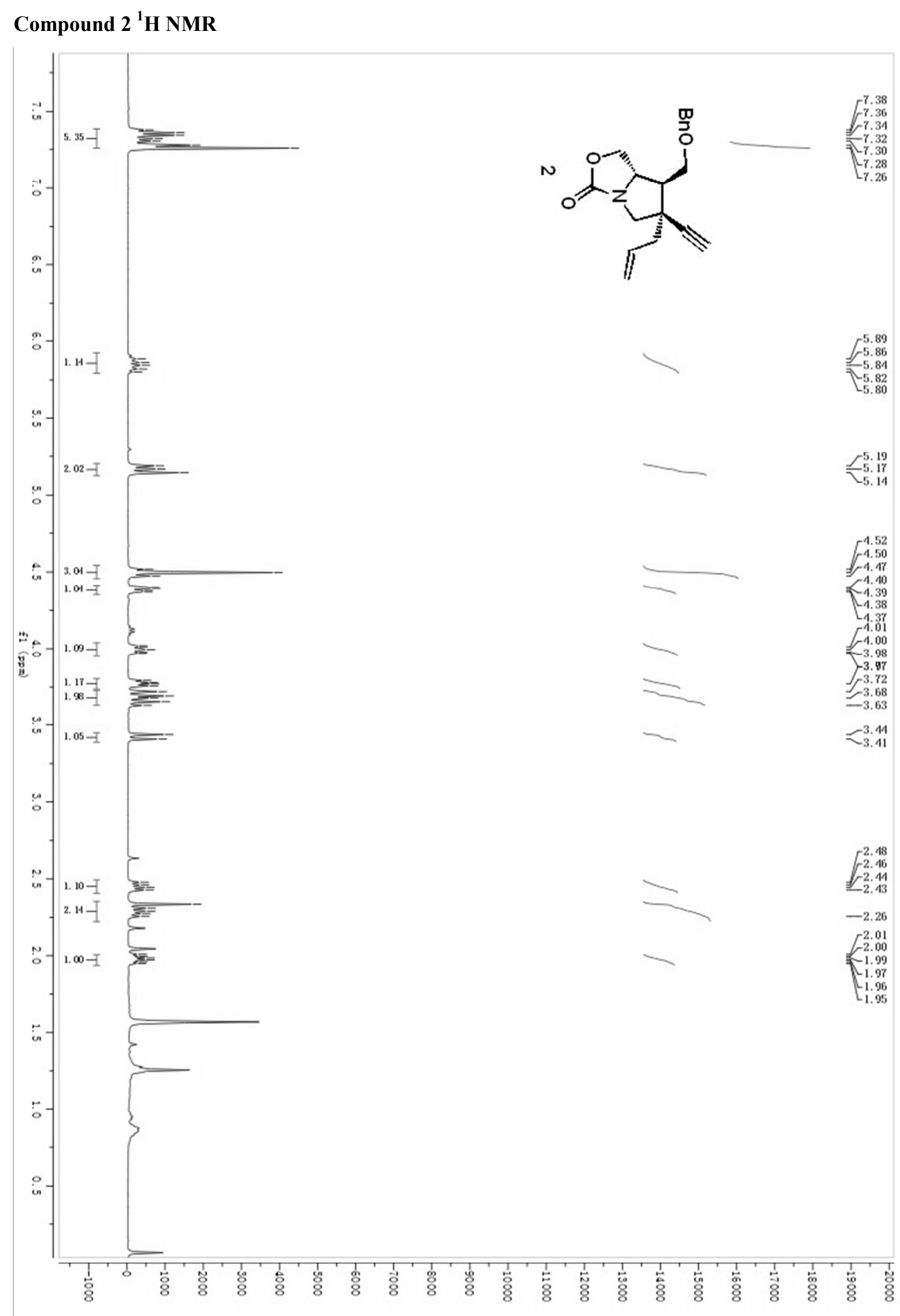


Compound $2{ }^{13} \mathrm{C}$ NMR

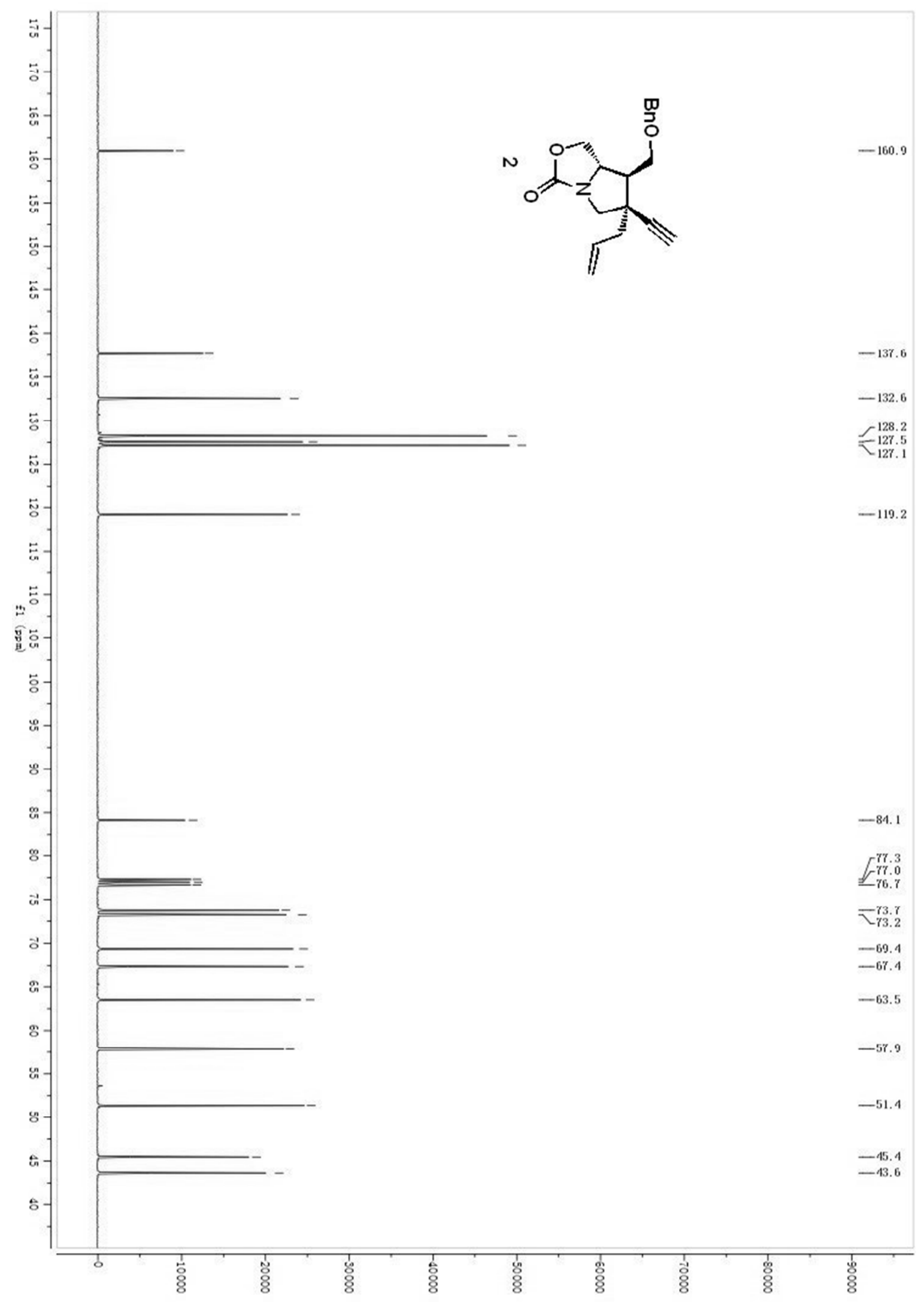




\section{Compound $17{ }^{1}$ H NMR}

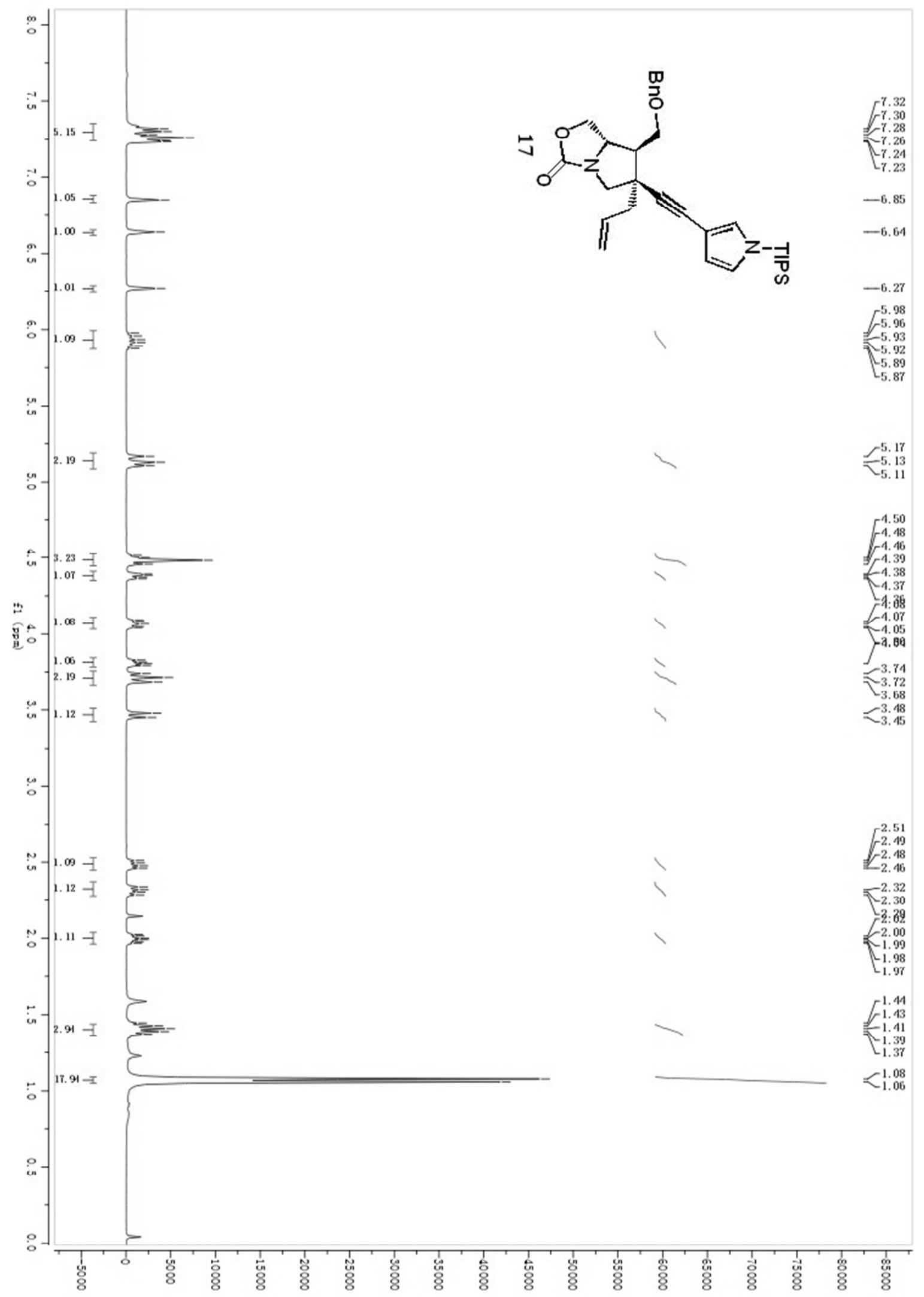


Compound $17{ }^{13} \mathrm{C}$ NMR

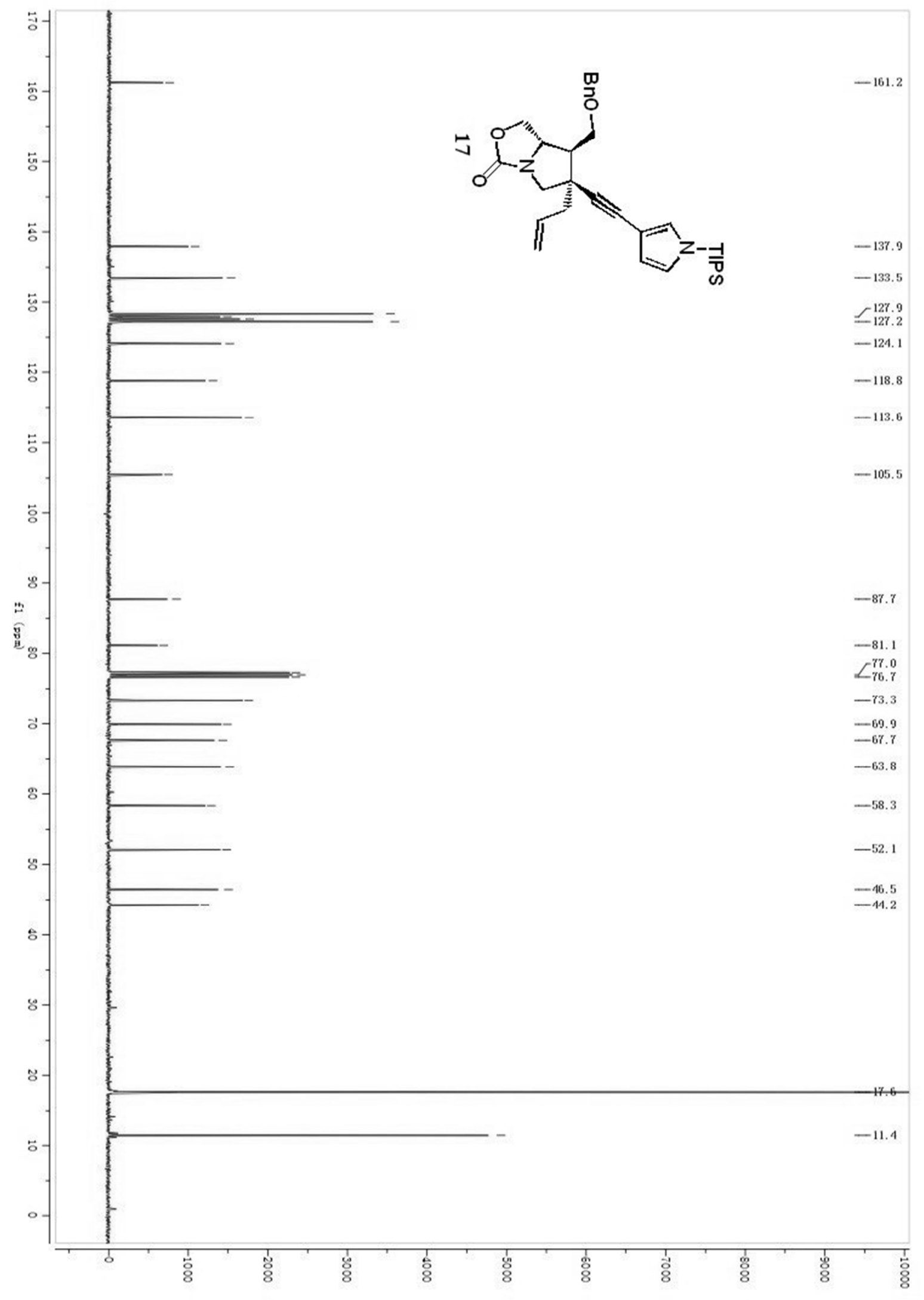




\section{Compound $18{ }^{1}$ H NMR}

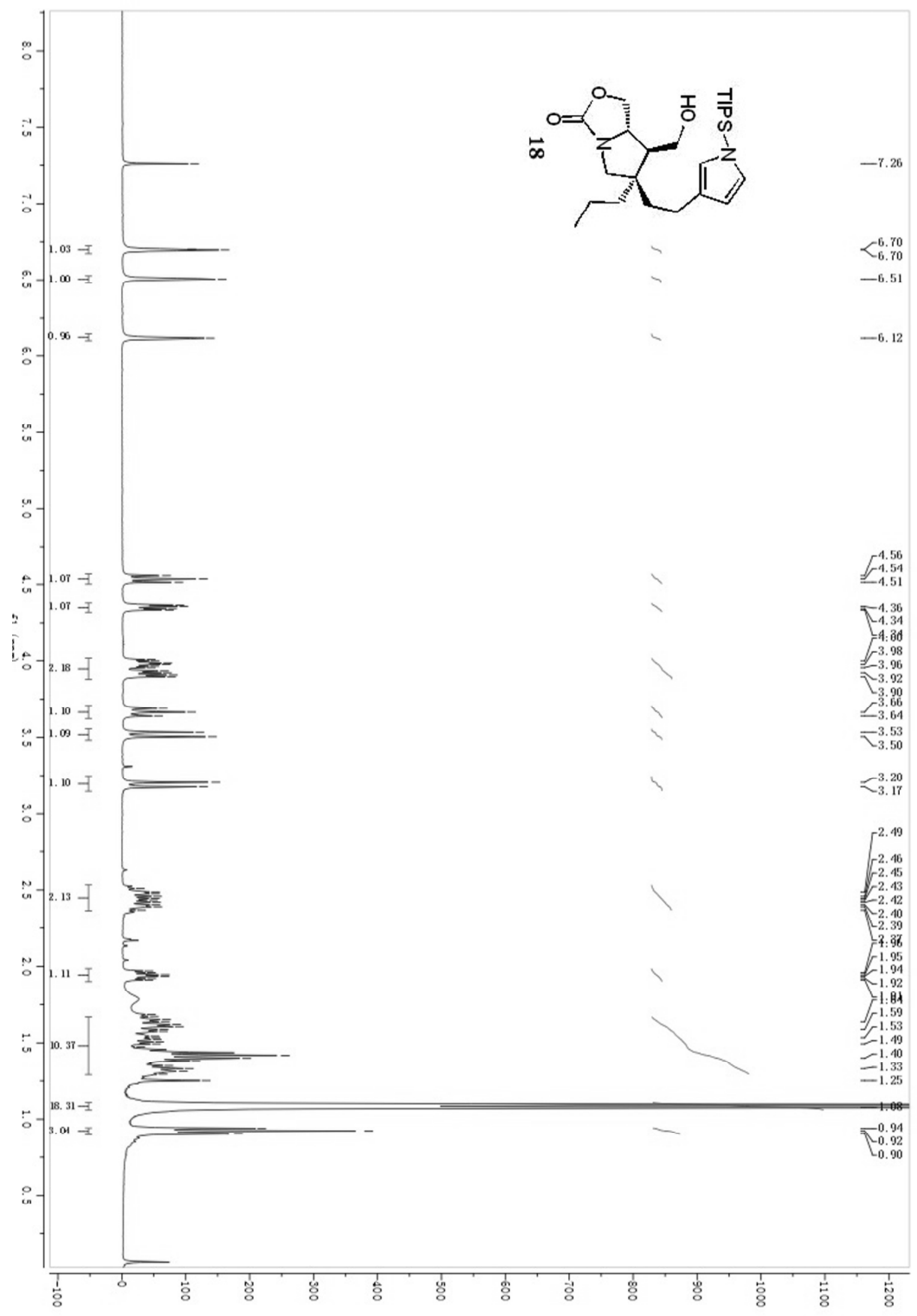




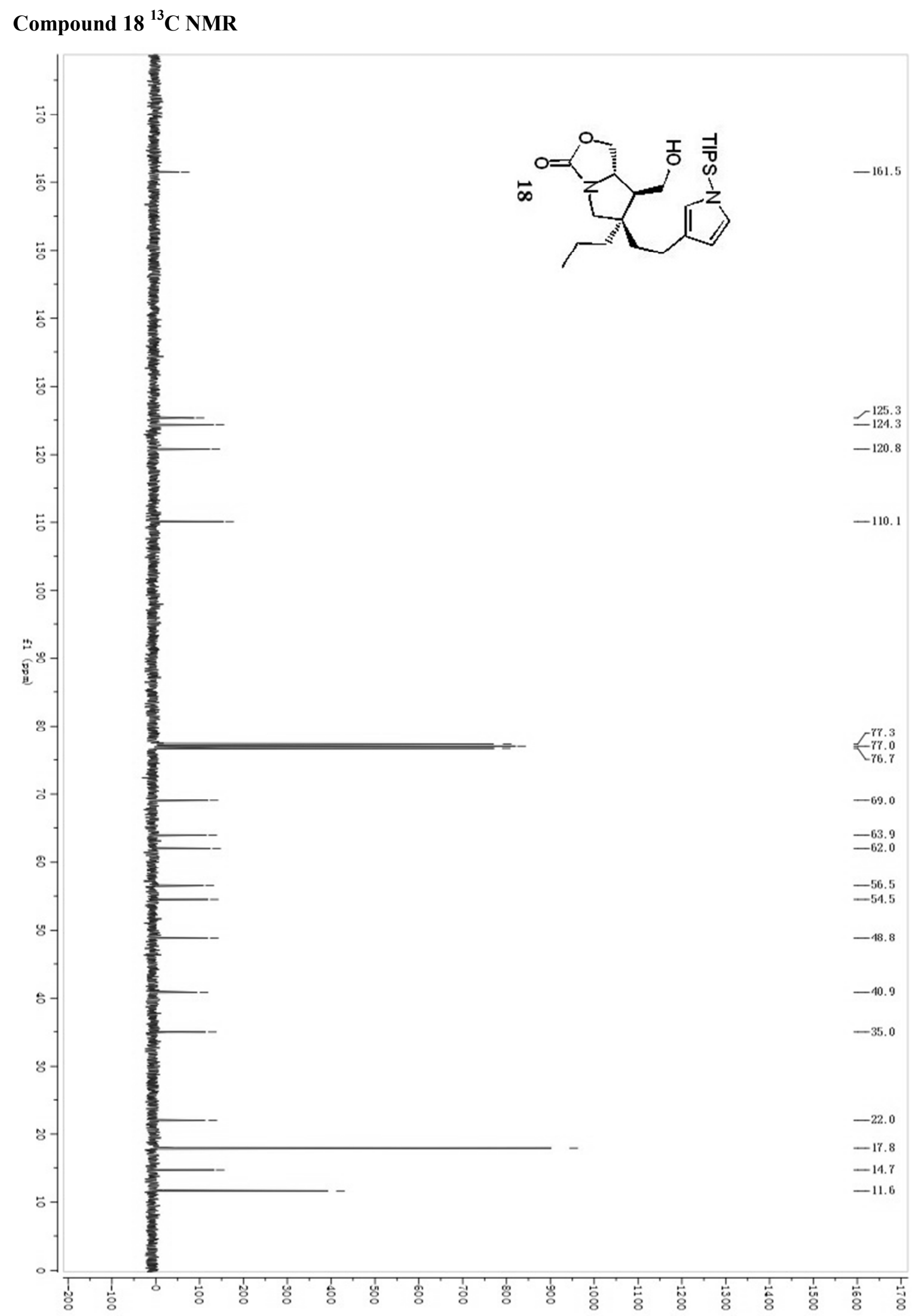




\section{Compound $19{ }^{1}$ H NMR}

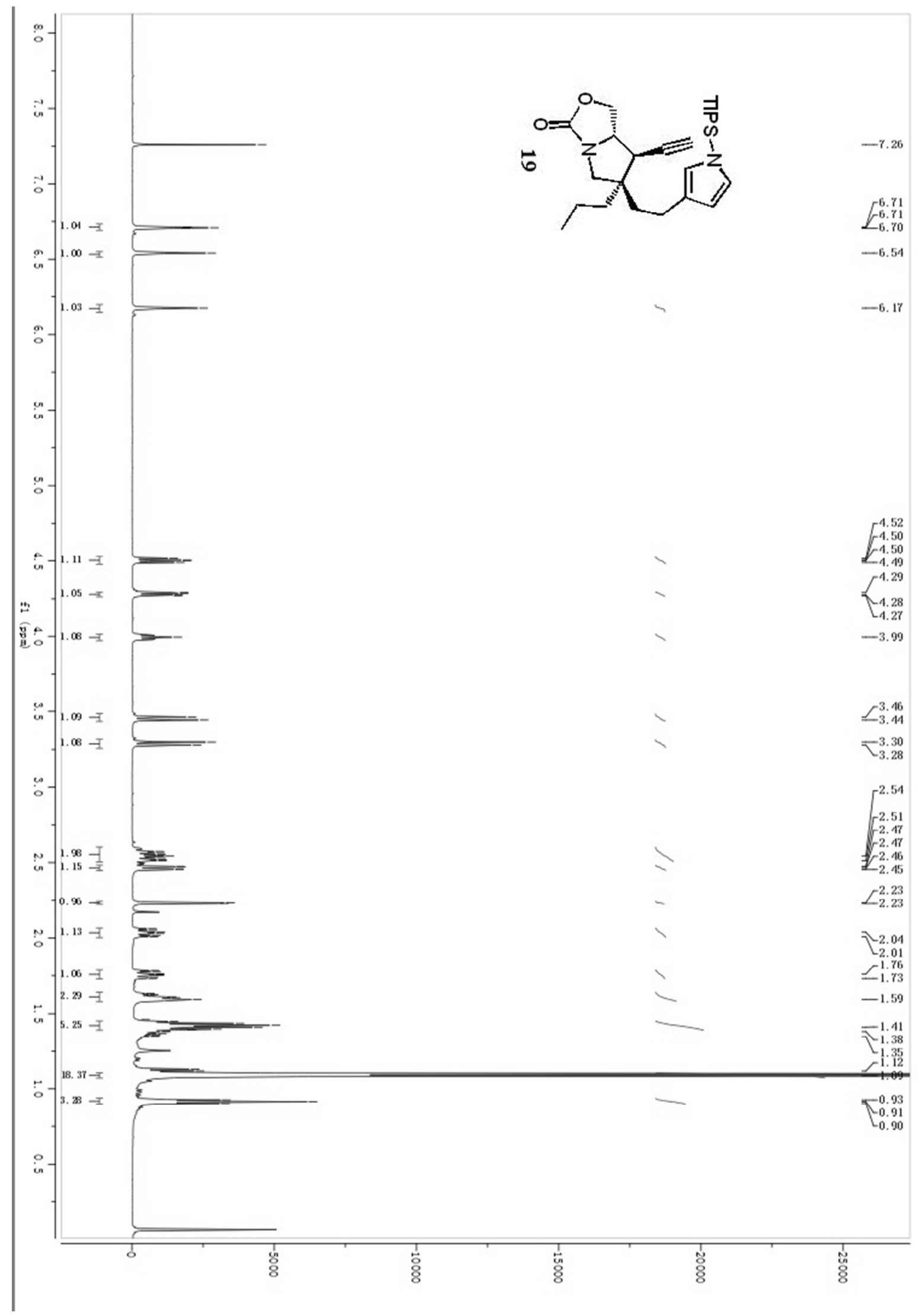




\section{Compound $19{ }^{13} \mathrm{C}$ NMR}

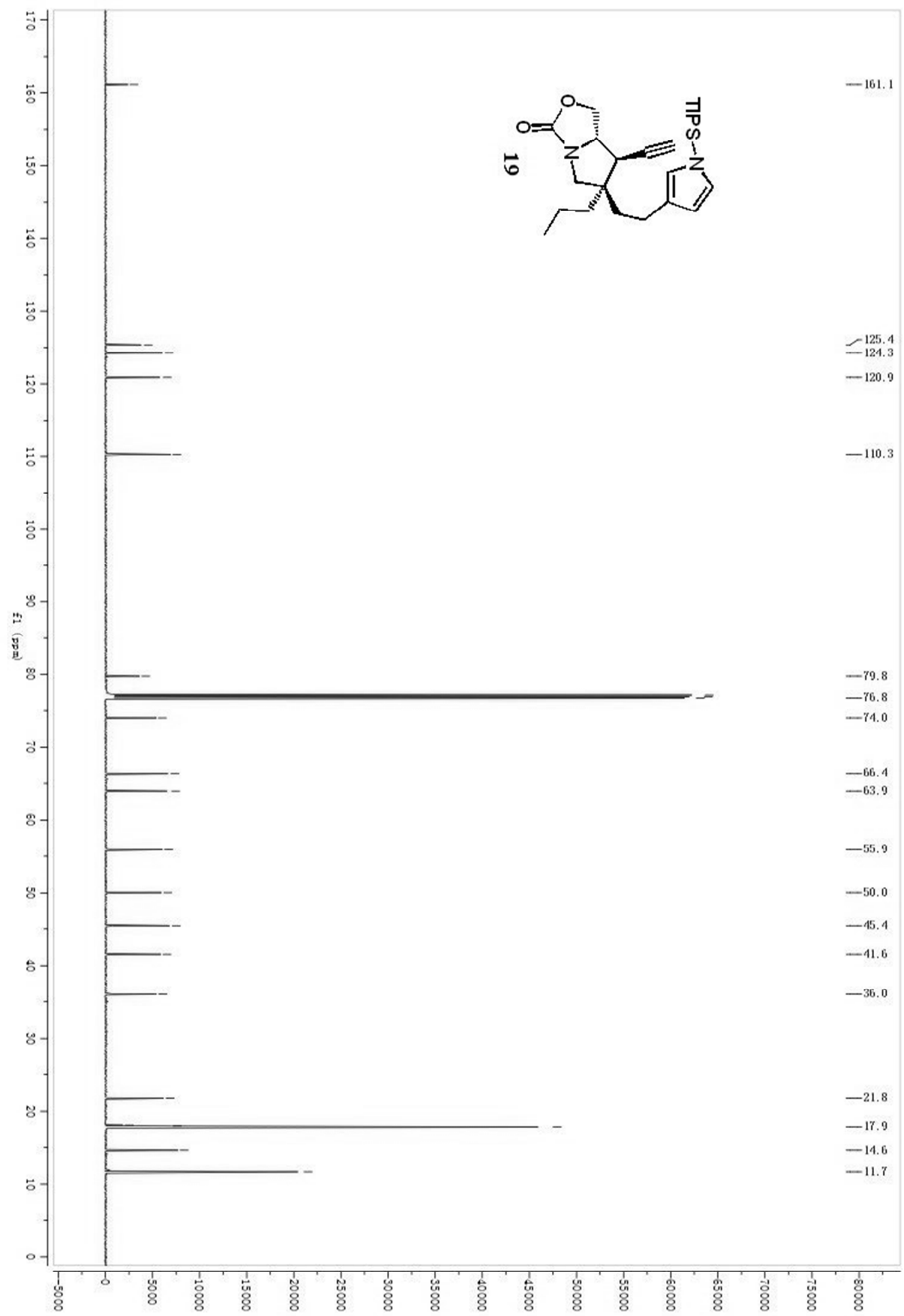




\section{Compound $20{ }^{1}$ H NMR}

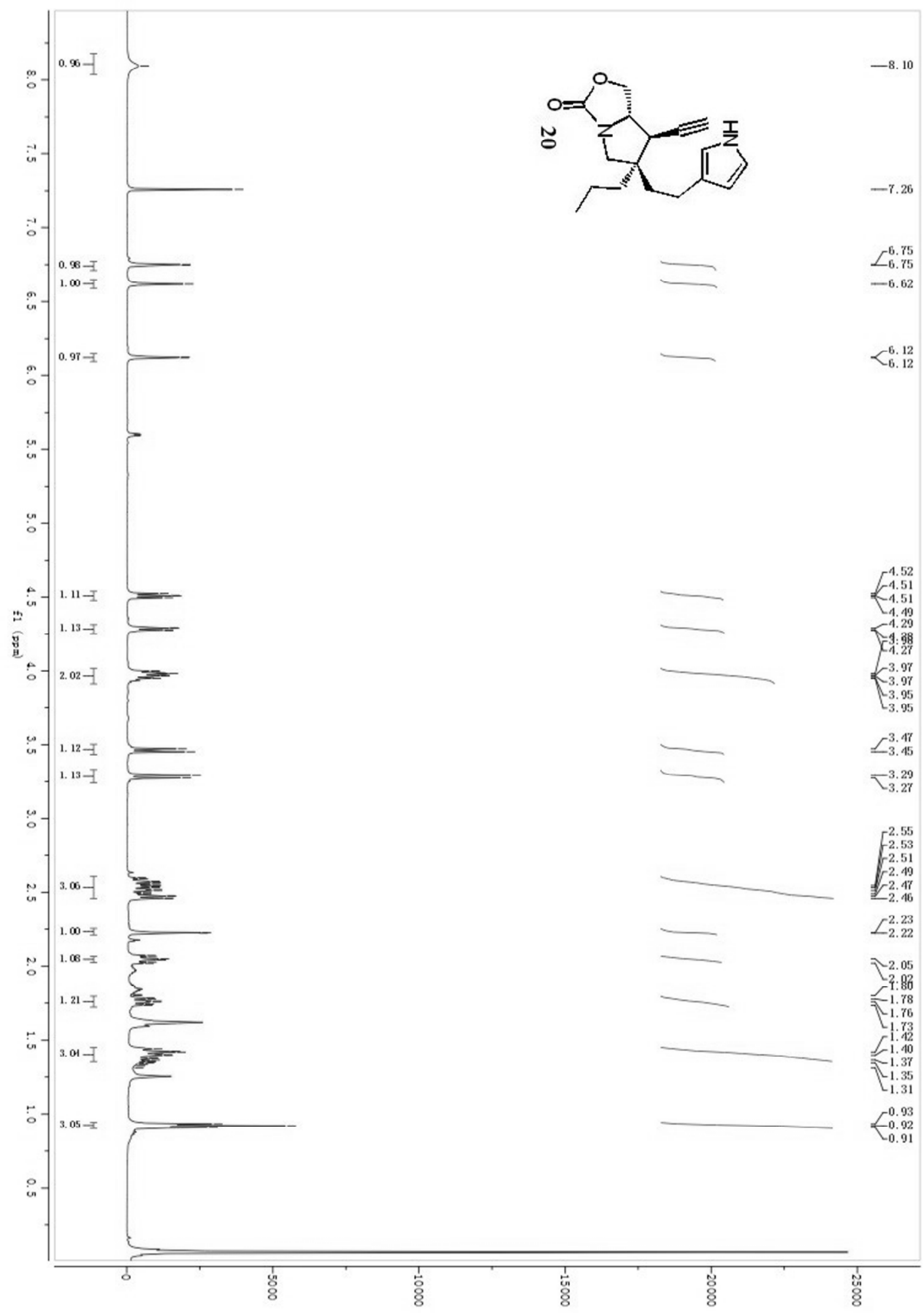




\section{Compound $20{ }^{13} \mathrm{C}$ NMR}

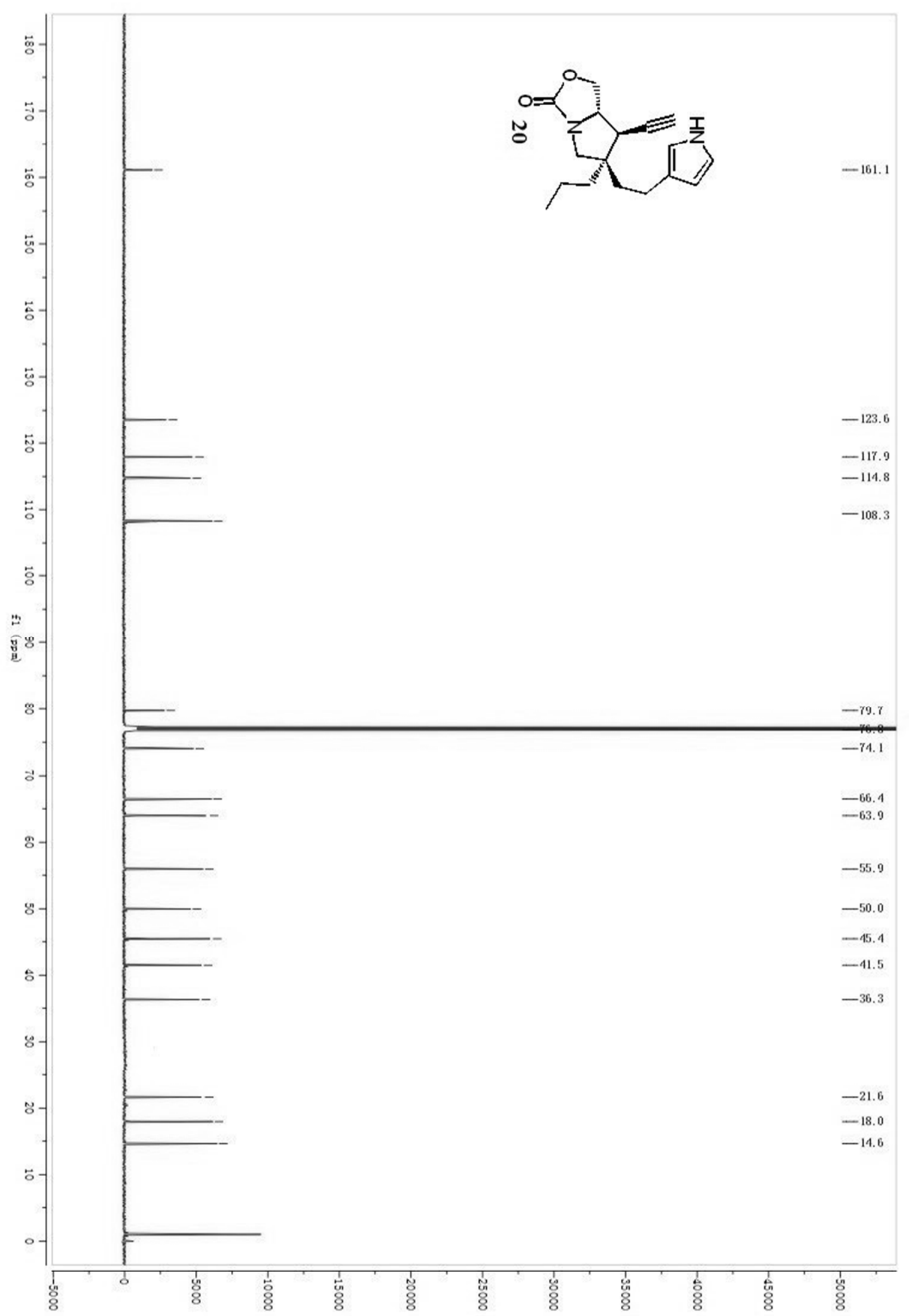




\section{Compound $1{ }^{1}$ H NMR}

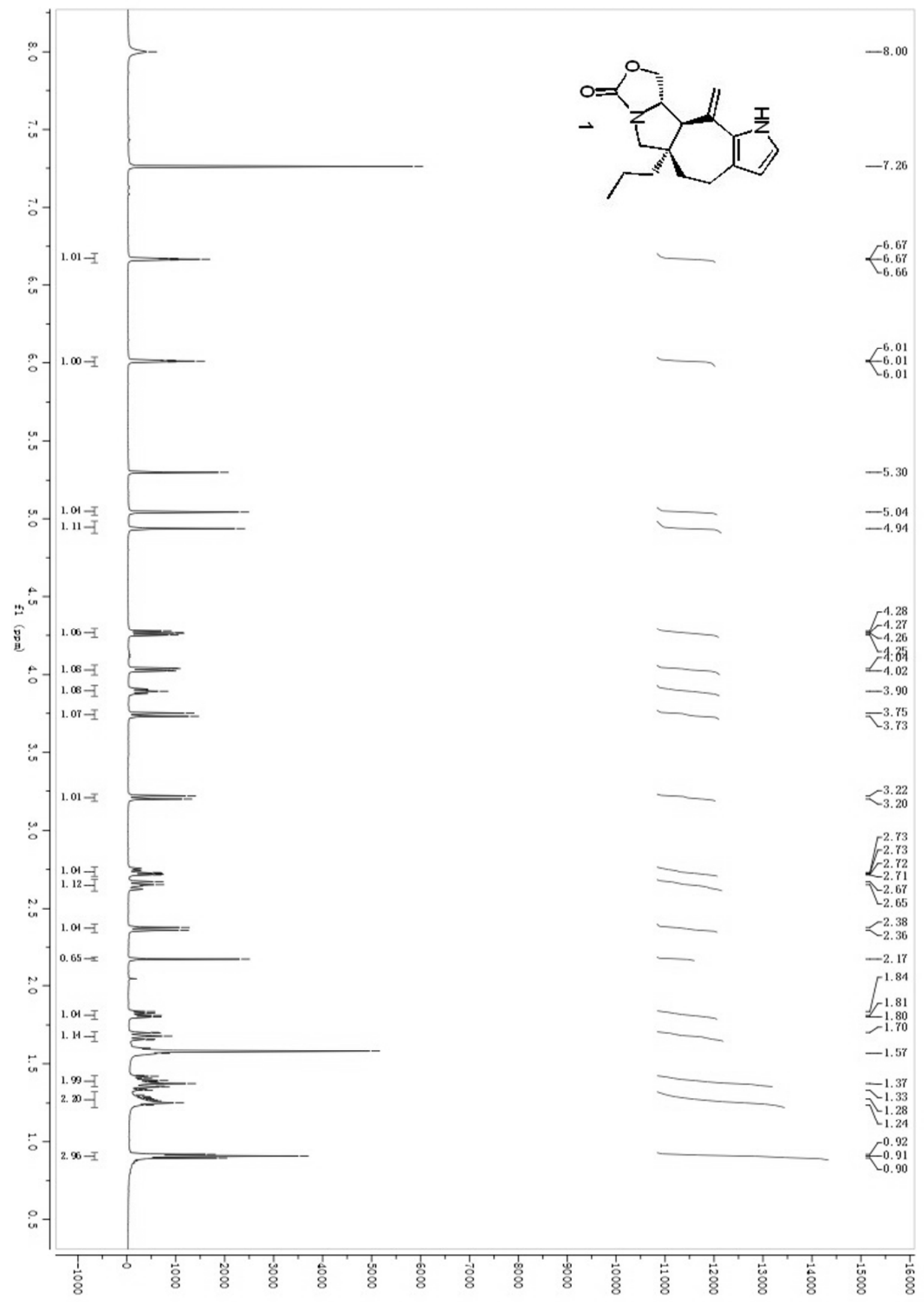


Compound $1{ }^{13} \mathrm{C}$ NMR

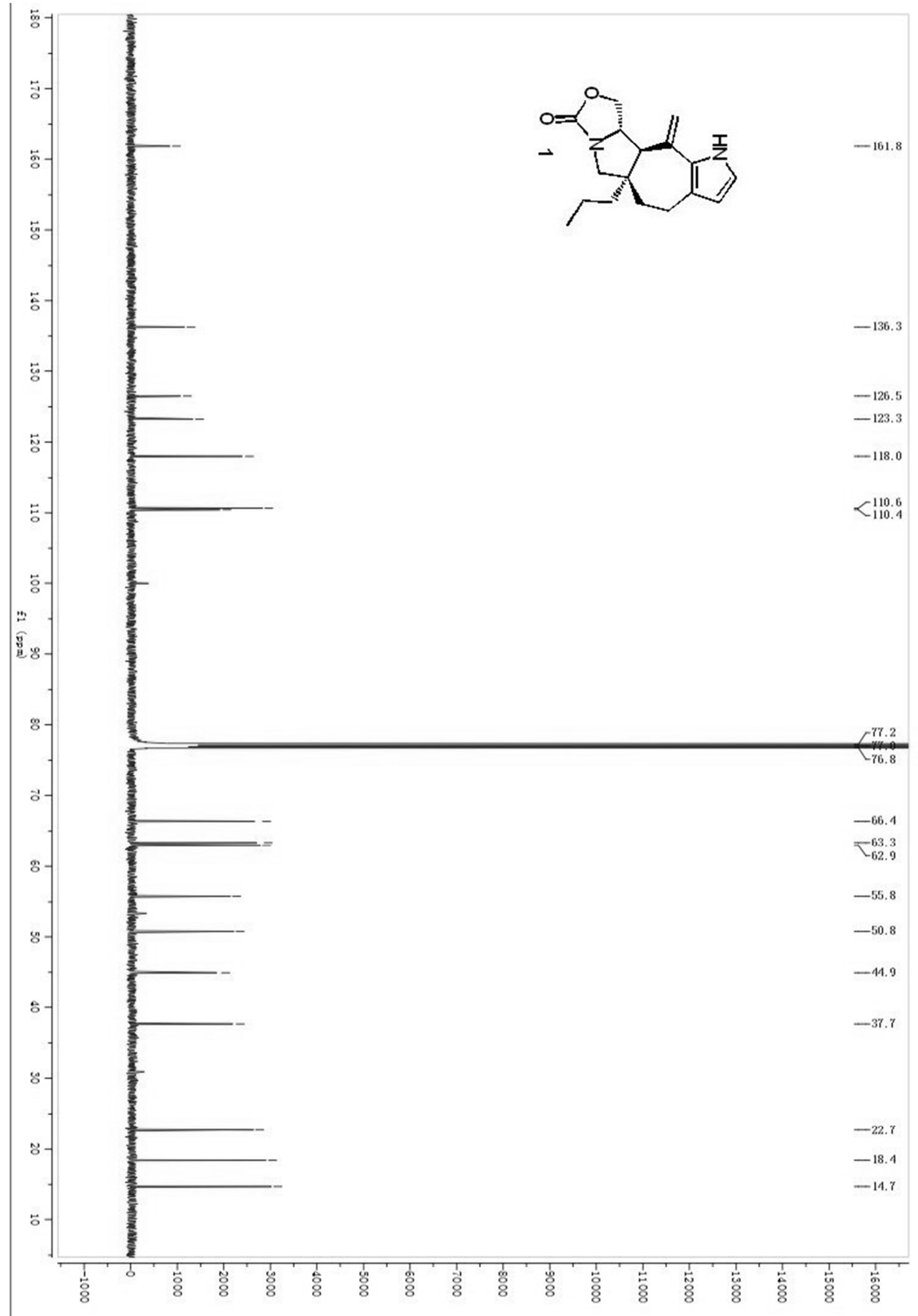

